UNIVERSIDAD

NACIONAL

DE LA PLATA

\title{
Mamíferos introducidos en Tierra del Fuego, el caso del peludo (Chaetophractus villosus): distribución, dieta, parasitología e impacto en especies autóctonas
}

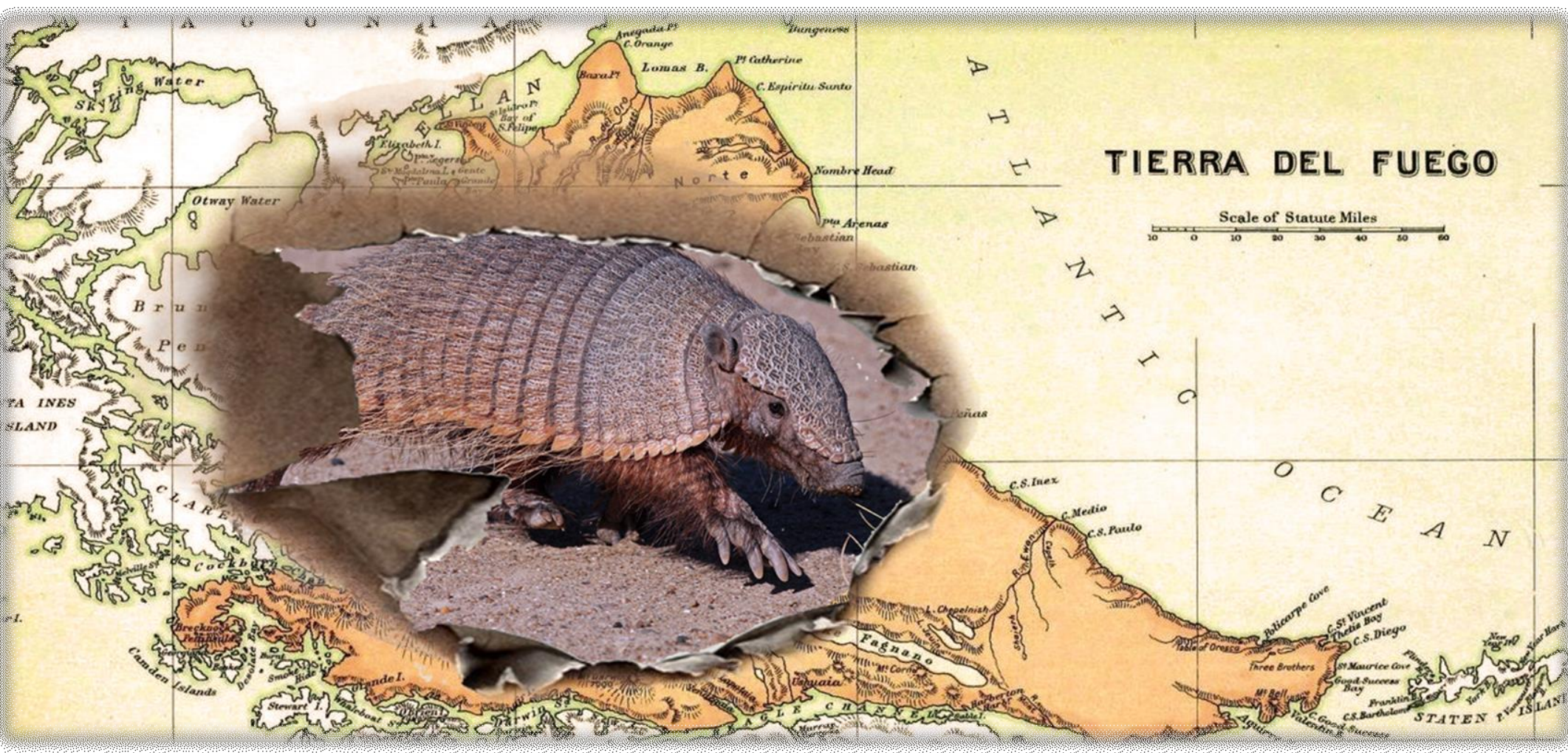

Lic. Jorge Alberto Gallo

Directores: Dr. Agustín Manuel Abba - Dra. Laura Fasola

Trabajo de Tesis para optar por el título de Doctor en Ciencia Naturales

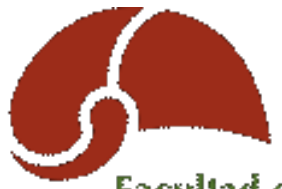

Factilad de Cisncias

Naturales y Museo 
"People can take everything away from you But they can never take away your truth But the question is, can you handle mine?..."

My Prerogative - B.J.S.

"La gente puede quitarte todo Pero nunca pueden quitarte tu verdad, la pregunta es, ¿'Podes con mi verdad?..."

My Prerogative - B.J.S. 


\section{AGRADECIMIENTOS}

Si bien debo reconocer que esta tesis fue desarrollada sin demasiadas complicaciones, en el camino pasaron muchas cosas que me ayudaron a continuar y a seguir amando esta profesión que elegí.

Agradezco eternamente a mis padres y hermanas, a quienes amo fuerte fuerte, por apoyarme siempre y proporcionarme los medios necesarios para nutrirme de la biología.

A mis abuelos, a quienes extraño y amo mucho, que a pesar de no entender mucho del tema siempre estuvieron orgullosos de su nieto biólogo.

A mis dos directores que me compartieron todos sus conocimientos, me acompañaron a perseguir peludos en las campañas y me apoyaron durante este trayecto.

Al rulo Abba, un groso de los xenartros, un incansable corredor de peludos, a veces un poquito pesado, pero siempre para bien, y que a pesar de las idas y vueltas siempre creyó en mí.

A lalita Fasola, que me movilizo desde Ushuaia a Bariloche, y me acompañó mostrándome toda la inmensidad de la Patagonia. Una genia de la estadística y de la ecología de invasiones, que me hizo buscar visones hasta en el freezer de mi heladera.

A las Dras. Cecilia Ezquiaga y Tatiana Rios unas parasitólogas muy copadas que me enseñaron mucho sobre esta relación parasitos-xenartros, aportaron material para esta tesis y con las que siempre quiero tomar una birra.

A la Dra. Graciela Navone y a todes les pibes de los laboratorios de parasitología del CEPAVE, que, a pesar de ser externo al instituto, me brindaron un lugar y toda la buena onda que tiene que tener un laboratorio durante el primer año de esta tesis.

A Olga, Mariano, Solsito, Nai, Rubia, Maru, Cecil, Anto y Meli porque los amo y a pesar de las vueltas de la vida, siempre estuvieron ahí para lo que necesité durante el recorrido de esta carrera de posgrado.

A Olga, que es más que una amiga, es mi todo. Aunque siempre añorando tomar una birra juntos y hablar pavadas, sus palabras y su manera de creer en mí que ayudaron en cada momento de esta tesis y de mi vida.

A Gastón, mi compañero de vida, a quien amo mucho y se hizo todo un experto en la invasión del peludo en Tierra del Fuego. 
Al Teatro independiente y sus belles seres, porque me permitió encontrar un escape mental, físico y emocional durante el desarrollo de esta tesis.

A la chechu love, florcha y Franco que durante los primeros años de esta tesis me dieron asilo, movilidad y mucho teatro.

A tute y nacho por altas jodas, lindos momentos compartidos y por seguir estando a mi lado aunque estemos lejos (sepan que no se merecen un agradecimiento tan formal).

A alita, la corri, debi, nati y eva, amigas del corazón que estuvieron conmigo desde que llegué a La Plata

A Fer, mi amiga y hermana del alma, que con toda su buena onda y energía mexicana me dio las mejores charlas y momentos llenos de música y chisme en el laboratorio, siempre teniendo presente que si "Britney pudo superar el 2007, nosotros también podíamos superar cada día".

A Tiño, que fue mi primer amigo "biólogo" que conocí en Bariloche y juntos siempre nos quejamos del explotado laburo de becario jaja.

Al CENAC (Centro de Estudios Aplicados a la Conservación del Parque Nacional Nahuel Huapi) que me dio un lugarcito hermoso en sus instalaciones para poder analizar mis muestras, escribir esta tesis y conocer personas excelentes.

Al Programa Patagonia de Aves Argentinas, por brindarme toda la logística para mis campañas y porque no solo me permitió conocer la Patagonia salvaje sino a personas increíbles.

A la martu y a Naty, amigas bellas, porque tuvimos la campaña fueguina "más normal" y más divertida de todas (y las que todavía nos quedan).

A la Vero por brindarme un hogar y compañía hermosa durante mi corta estadía en Ushuaia.

A Gabo Martin y Dani Udrizar Sauthier por abrirnos las puertas de sus casas, por aportar parte de las muestras y por acompañarnos a recorrer parte de la Patagonia.

Al Consejo Nacional de Investigaciones Científicas y Técnicas (CONICET) por otorgarme una beca para poder realizar mi doctorado.

A la Facultad de Ciencias Naturales y Museo de la U.N.L.P que me brindo todo el conocimiento y me acercó a hermosas personas. 
Y a los armadillos, que sin pensarlo se convirtieron en los mamíferos más lindos e interesantes que existen. 


\section{RESUMEN}

Una especie exótica invasora es aquella que logra establecerse y dispersar fuera de su rango de distribución natural y que puede afectar la riqueza y abundancia de las especies nativas de un ecosistema a través de interacciones directas, como la depredación, o indirectas, alterando la disponibilidad de recursos, modificando la composición de las comunidades y/o alterando la dinámica de enfermedades de las especies nativas. Así mismo, toda invasión biológica involucra tanto aspectos ecológicos como sociales, siendo la dimensión humana un variable importante al momento de estudiar el proceso de invasión de una especie introducida.

Chaetophractus villosus es un armadillo omnívoro-carnívoro de hábitos semifosoriales que se encuentra ampliamente distribuido en Argentina. Sin embargo, su presencia en Patagonia continental fue detectada hace aproximadamente 100 años como resultado de una dispersión facilitada por el hombre, y en la década de 1980 fue introducido en la Isla Grande de Tierra del Fuego.

El objetivo de la presente tesis es obtener información actualizada acerca de la distribución, dieta e impacto sobre especies nativas de Chaetophractus villosus en la Isla Grande de Tierra del Fuego. A su vez, se analizó la diversidad y abundancia de helmintos parásitos de C. villosus y Zaedyus pichiy para comprender las variaciones en la comunidades parásitas entre la Patagonia continental (Chubut y Santa Cruz) y localidades al norte del Río Chubut y el evento de introducción en Tierra del Fuego.

Se recorrieron 52 transectas en la estepa y parte del ecotono de la Isla Grande de Tierra del Fuego en busca de evidencias indirectas de peludos. Se evaluaron las asociaciones con el ambiente según la presencia de armadillos (ocurrencia) y el número de evidencias (uso del espacio). Se construyó un mapa de calor para visualizar áreas de mayor actividad de armadillos y así identificar zonas donde deben implementarse planes de manejo y monitoreo de la invasión.

Se realizaron encuestas $(n=20)$ a pobladores o trabajadores locales del departamento de Río Grande en la Isla Grande de Tierra del Fuego para conocer la percepción social de la invasión del peludo en la isla. 
Para el estudio de dieta se colectaron 34 individuos atropellados de C. villosus en Chubut y Santa Cruz, a su vez se colectaron y sacrificaron 12 individuos en la Isla Grande de Tierra del Fuego, se analizaron los contenidos estomacales y se realizaron las comparaciones pertinentes. Se evaluó la depredación del peludo sobre aves que nidifican en el suelo en Tierra del Fuego a partir de nidos artificiales y cámaras trampa.

Se colectaron y prospectaron 49 sistemas digestivos de armadillos hospedadores: 23 Chaetophractus villosus de Patagona continental, 12 C. villosus de Tierra del Fuego y 14 Zaedyus pichiy de Patagonia continental. Los helmintos fueron estudiados mediante técnicas convencionales y posteriormente se realizaron análisis ecológicos a nivel de comunidad componente, población componente y se evaluó la estructura comunitaria.

La distribución de C. villosus se incrementó $4736 \mathrm{~km}^{2}$ desde el último estudio publicado. Actualmente es frecuente en toda la estepa (parte norte de la Isla Grande) y ocupa también gran parte del ecotono fueguino. Es más probable encontrar peludos en zonas elevadas no inundables, con una altura de la vegetación media-alta (mayor a $30 \mathrm{~cm}$ ) y con suelos de tipo húmico (con alto contenido de materia orgánica). No se encontraron asociaciones entre la presencia de peludos y la distancia a característica del paisaje tales como gasoductos, ríos, costa marina o asentamientos humanos.

En Tierra del Fuego la tasa de expansión del peludo en la Isla Grande fue de 10,9 km/año y si bien la dispersión hacia el sur de la isla se vio limitada por dos ríos (Río Chico y Río Grande), los resultados de las encuestas realizadas en esta tesis permitieron comprobar que la traslocación de individuos sería uno de los mecanismos por los cuales este armadillo logró traspasar barreras naturales como los ríos.

Se pudieron distinguir dos zonas de mayor actividad de peludos donde deberían llevarse a cabo planes de manejo de la especie invasora. Una zona A, coincidente con el sitio de introducción, y una zona $B$, donde este armadillo logró establecerse más recientemente y constituye el frente sur de la invasión.

El principal uso del peludo por la población local es para consumo. Así mismo es considerado dañino en los sistemas agropecuarios principalmente porque la construcción de cuevas representa un riesgo para el ganado. 
La diversidad de la dieta del peludo en Tierra del Fuego es más baja que en su distribución nativa (Patagonia continental). Las larvas de "Scarabaeidae" y "Restos vegetales" son los ítems dominantes en la población invasora. La evaluación de depredación sobre aves que nidifican en el suelo no permite concluir que el peludo deprede activamente sobre huevos de aves en la Isla. Sin embargo, la presencia de $C$. villosus en la Isla Grande de Tierra del Fuego como un mamífero omnívoro-carnívoro, sumaría una nueva especie al ensamble de mamíferos exóticos presentes en la zona, haciendo aún más compleja la situación poblacional de las potenciales presas nativas de la Isla.

Los estudios parasitológicos en las dos especies de armadillos patagónicos evidenciaron una disminución de la diversidad de helmintos de aproximadamente el 50\%, respecto a las poblaciones al norte del Río Chubut para ambos hospedadores. Para $Z$. pichiy esta disminución podría deberse a la baja tolerancia de determinados parásitos a las condiciones climáticas que presenta la Patagonia centro-sur (sobre todo estadíos larvales) y la disminución de la riqueza y la diversidad de artrópodos que operan como hospedadores intermediarios para muchos parásitos. Sin embargo, la estructura comunitaria de la población patagónica es muy similar a la encontrada al norte de la distribución. Para el caso de C. villosus, la ausencia de ciertos parásitos con distribución patagónica, como el cestode Mathevotaenia sp., y la gran disminución de la prevalencia de ciertos helmintos respecto a la región núcleo (región pampeana), podrían ser el resultado de la reciente dispersión del peludo hacia el sur del Río Chubut. Así mismo para Tierra del Fuego, el parásito Trichohelix tuberculata es la única especie presente en la población invasora de $C$. villosus, al igual que lo registrado en el último estudio realizado. A su vez, se encontraron nuevos registros de parásitos para Patagonia, lo que permite ampliar los rangos de distribución geográfica de los nematodes Aspidodera fasciata, $A$. scoleciformis, Pterygodermatites argentinensis, Strongyloides sp. y del cestode Mathevotaenia sp. y se fija la provincia de Santa Cruz como el nuevo límite austral de sus distribuciones. 
Los resultados de esta tesis permiten concluir que el peludo se encuentra invadiendo toda la estepa y gran parte del ecotono de la Isla Grande de Tierra del Fuego. En este contexto, esta invasión debe considerarse como un problema ecológico y social que necesita ser resuelto a través de medidas que impidan el avance de nuevas áreas de la Isla Grande de Tierra del Fuego. 


\section{ABSTRACT}

Invasive Alien Species are those that become established and spread outside their native range due to human actions. Alien species may affect richness and abundance of native species through direct interactions (predation) or indirect interactions (modifying community structure, resources availability or changing the dynamic of diseases of native species). In the same way, any biological invasion involves not only ecological aspects but social aspects too. In this context, human dimension is an important aspect to consider in any study of an introduced species.

Chaetophractus villosus is an omnivorous-carnivorous armadillo with semi-fosorial habits widespread in Argentina. This armadillo is present in continental Patagonia since 100 years ago as result of a spread facilitated by human and was introduced in the Isla Grande of Tierra del Fuego in the 1980 decade.

The objective of this thesis is to update information of distribution, ecology and impact on native species of $C$. villosus in the Isla Grande of Tierra del Fuego. The abundance and diversity of parasites helmints of C. villosus and Zaedyus pichiy were analyzed to understand variations in parasite community between Patagonia (including Tierra del Fuego) and localities at northern of Chubut river.

Fifty two transects were conducted in the steppe and ecotono of the Isla Grande of Tierra del Fuego looking for indirect evidences of $C$. villosus. We evaluated the association of each habitat characteristic with the presence of armadillos (Occurrence analysis) and with the number of signs of presence (Intensity of use analysis). A heat map was developed to visualize those areas of high armadillo's activity and thus identify areas for control/eradication of this invader and monitor its expansion.

Interviews ( $n=20)$ with ranch settlers and workers of the Rio Grande department of Tierra del Fuergo were conducted to analyze the social perception of the invasion process. For diet analysis 34 road-killed individuals of $C$. villosus were collected in Santa Cruz and Chubut provinces and 12 C. villosus were captured and euthanized in Tierra del Fuego. Predation analysis on ground nesting birds were conducted using artificial nests and camera traps 
A total of 49 digestive systems of armadillos were analyzed: 23 C. villosus of continental Patagonia, 12 C. villosus of Tierra del Fuego and 14 Zaedyus pichiy of continental Patagonia. Helminths found were studied by conventional techniques. Ecological analyses were carried out at the level of the component community, component population and community structure.

The distribution of $C$. villosus in Tierra del Fuego increased $4736 \mathrm{~km}^{2}$ since the last study published. The species is now frequently seen in the northern part of the island. Our updated distributional data shows that the large hairy armadillo occurs throughout the steppe and also in the ecotone area. C. villosus is more likely to be found in medium and tall vegetation with humic and not floodable soils. No associations with landscape features (oil pipes, marine coast, rivers, humans settlements) were found.

The expansion rate estimated for C. villosus in TDF was $10.9 \mathrm{~km} / \mathrm{year}$ and even the spread of this armadillo through the Isla Grande has been limited principally by rivers (Rio Chico y Rio Grande). The results of the interviews showed that these natural barriers may not have been completely impassable because of the translocations of armadillos by humans.

We identified two areas on Isla Grande where an action plan should be focused. Area " $A$ " is close to the original introduction points and area " $B$ " where armadillos were established more recently and is closer to the southern invasion front.

The main use of armadillos by local people was for food (Fig. 4b). As for negative impacts of $C$. villosus on rural activities, most of the interviewees said that burrows represented a risk of injury to the livestock if they step into the burrows.

Diet diversity in Tierra del Fuego is lower than in its native range. "Scarabaeidae" larvae and "plant material" are dominant in Tierra del Fuego. The predation analysis over ground-nesting birds does not showed that C. villous predate over birds eggs. But the precence of this omnivorous-carnivorous armadillo in the island adds another mammal to the enssable of alien mammal of Tierra del Fuego complicating the situation of native preys. 
Parasitological studies showed that both armadillos patagonian hosts lost $50 \%$ of helmints diversity. For Z. pichiy, the lower parasite diversity could be explained by the low tolerance of some parasites to harsh climatic conditions of southern Patagonia, or even to de absence of intermediate hosts. However, the community structure of pichi patagonian population was similar to those found in northern popultion. In C. villosus, the absence of some helmints species distributed in Patagonia and the differences on the community structures could be the results of the recent dispersal of this armadillo soutern to Chubut river. In Tierra del Fuego, the only parasite present was Trichohellix tuberculata, like was registered in the last study published. New records of patagonian parasites were found. Aspidodera fasciata, A. scoleciformis, Pterygodermatites argentinensis, Strongyloides sp. and the cestode Mathevotaenia sp expand their distribution range southern of Santa Cruz province.

The results of this thesis showed that the large hairy armadillo is present in the steppe and part of the ecotono of the Isla Grande of Tierra del Fuego. In this context is important to view this invasion as a socio- ecological problem that needs to be solved. 
Agradecimientos.

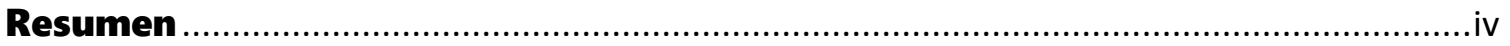

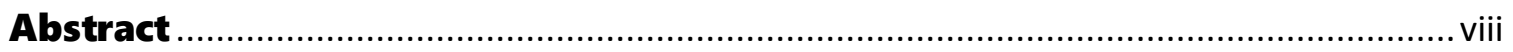

Capítulo 1: Introducción General ..............................................................1

1.1 Las invasiones biológicas como problemática ecológica y social................................... 2

1.2 Chaetophractus villosus dentro del magnorden xenarthra ......................................... 4

1.3 Área de estudio: la Isla Grande de Tierra Del Fuego ................................................... 6

1.4 Chaetophractus villosus en Tierra del Fuego: posibles impactos y antecedentes de

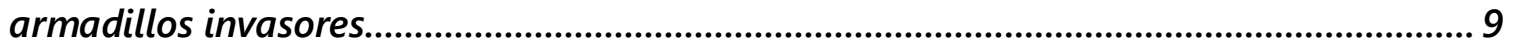

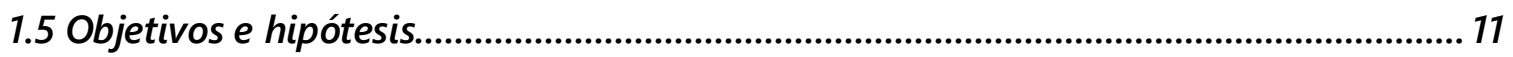

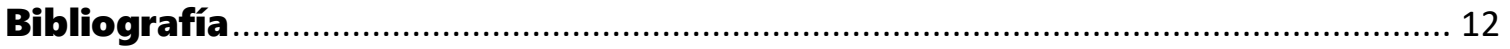

\section{Capítulo 2: Distribución y Uso del espacio del peludo} en Tierra del Fuego .................................................................................. 18

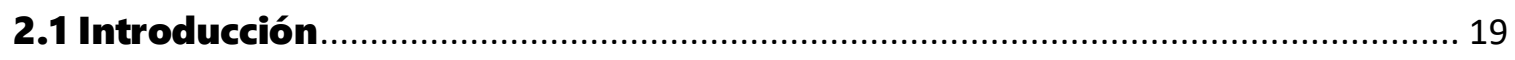

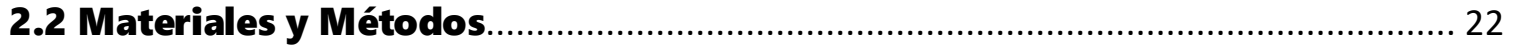

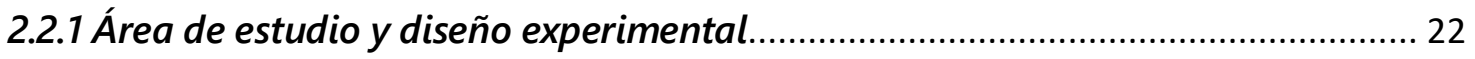

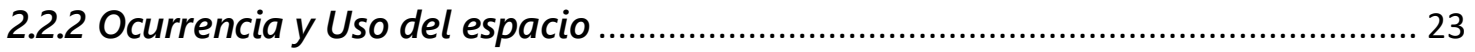

2.2.3 Influencia de la distancia a estructuras del paisaje en la presencia de la especie...........25

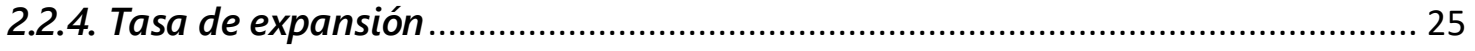

2.2.4. Áreas de concentración de presencia e intensidad de uso ................................. 25

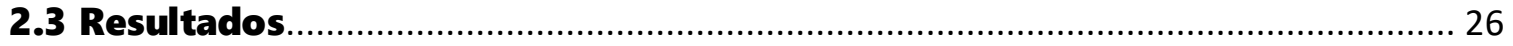

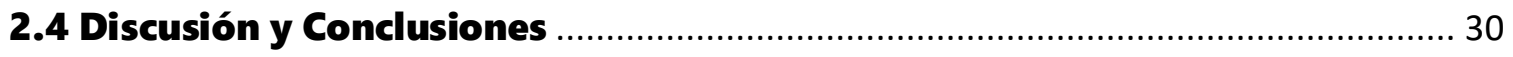

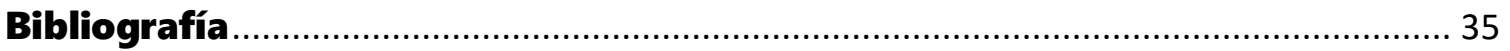

Capitulo 3: Percepción de la invasión .......................................................40

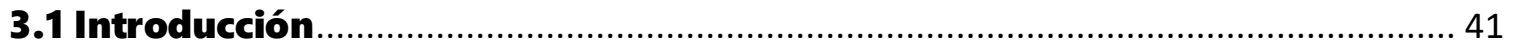

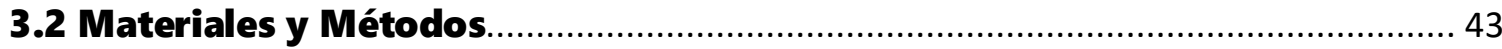

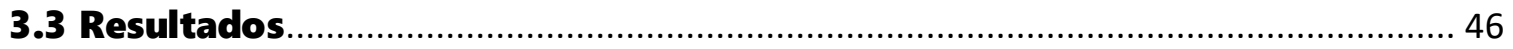




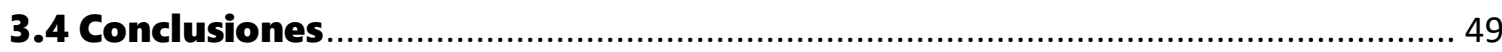

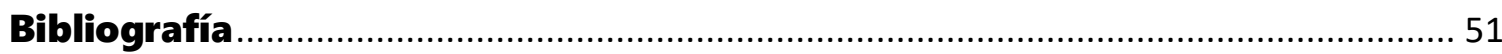

Capitulo 4: Hábitos alimenticios e impacto sobre especies autóctonas ....54

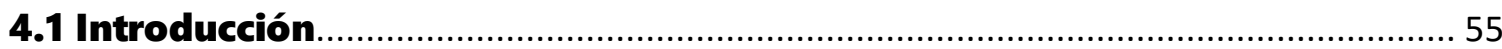

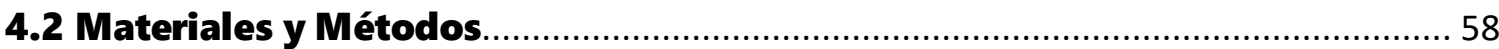

4.2.1 Análisis del contenido estomacal.............................................................. 58

4.2.2 Depredación de C. villosus sobre aves nativas ................................................... 60

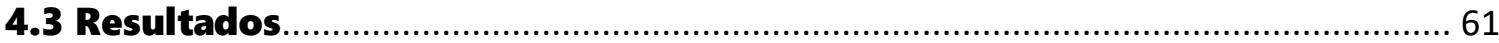

4.3.1 Dieta de Chaetophractus villosus en Patagonia continental ............................. 61

4.3.2 Dieta de Chaetophractus villosus en Tierra del Fuego ........................................ 63

4.3.3 Depredación de Chaetophractus villosus sobre aves nativas en Tierra del Fuego ........ 64

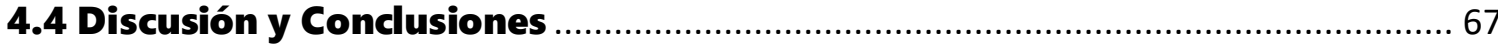

\section{Capitulo 5: Helmintos de armadillos de Patagónia: Co-introducción}

parásito-hospedador ...............................................................................73

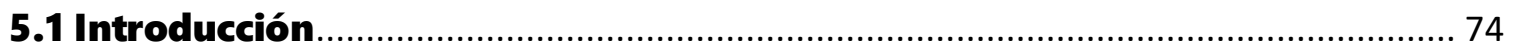

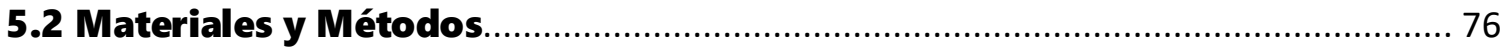

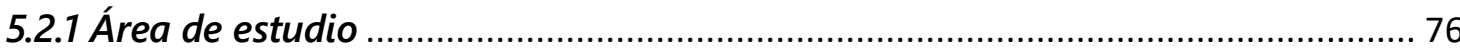

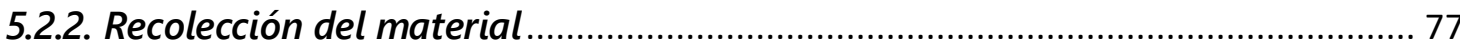

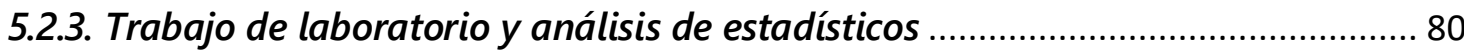

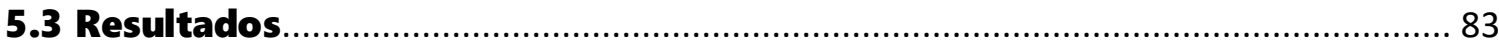

5.3.1 Análisis de la población y comunidad componente de helmintos para ambos hospedadores en Patagonia............................................................................................ 83

5.3.2 Estructura de la comunidad parasitaria............................................................. 84

5.3.3 Zaedyus pichiy: Población Norte vs. Población Patagonia Sur.......................... 86

5.3.4 Chaetophractus villosus: Población Núcleo vs. Población Patagonia Sur .......... 87

5.3.5 Chaetophractus villosus: Población TdF 2002-2006 vs. Población TdF Actual... 88

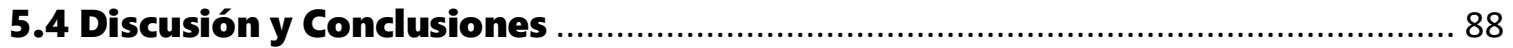

5.4.1 Variaciones en la helmintofauna entre poblaciones Norte y Patagonia Sur de

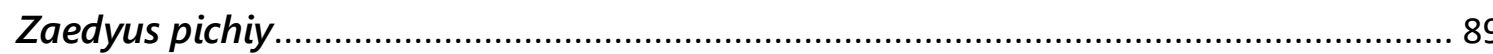


5.4.2. Variaciones en la helmintofauna de las poblaciones Núcleo y Patagónicas de Chaetophractus villosus

5.4.3 Estructura de la comunidad de helmintos de Zaedyus pichiy y Chaetophractus villosus

5.4.4. Nuevos registros de helmintos en Patagonia Sur 93

Bibliografía. 94

Capitulo 6: Conslusiones finales y recomendaciones de manejo 99

6.1 La invasión exitosa del peludo en Tierra del Fuego .100

6.2 Recomendaciones para el manejo del meludo en Tierra del Fuego. .101 Bibliografía .104 


\section{INTRODUCCIÓN GENERAL}

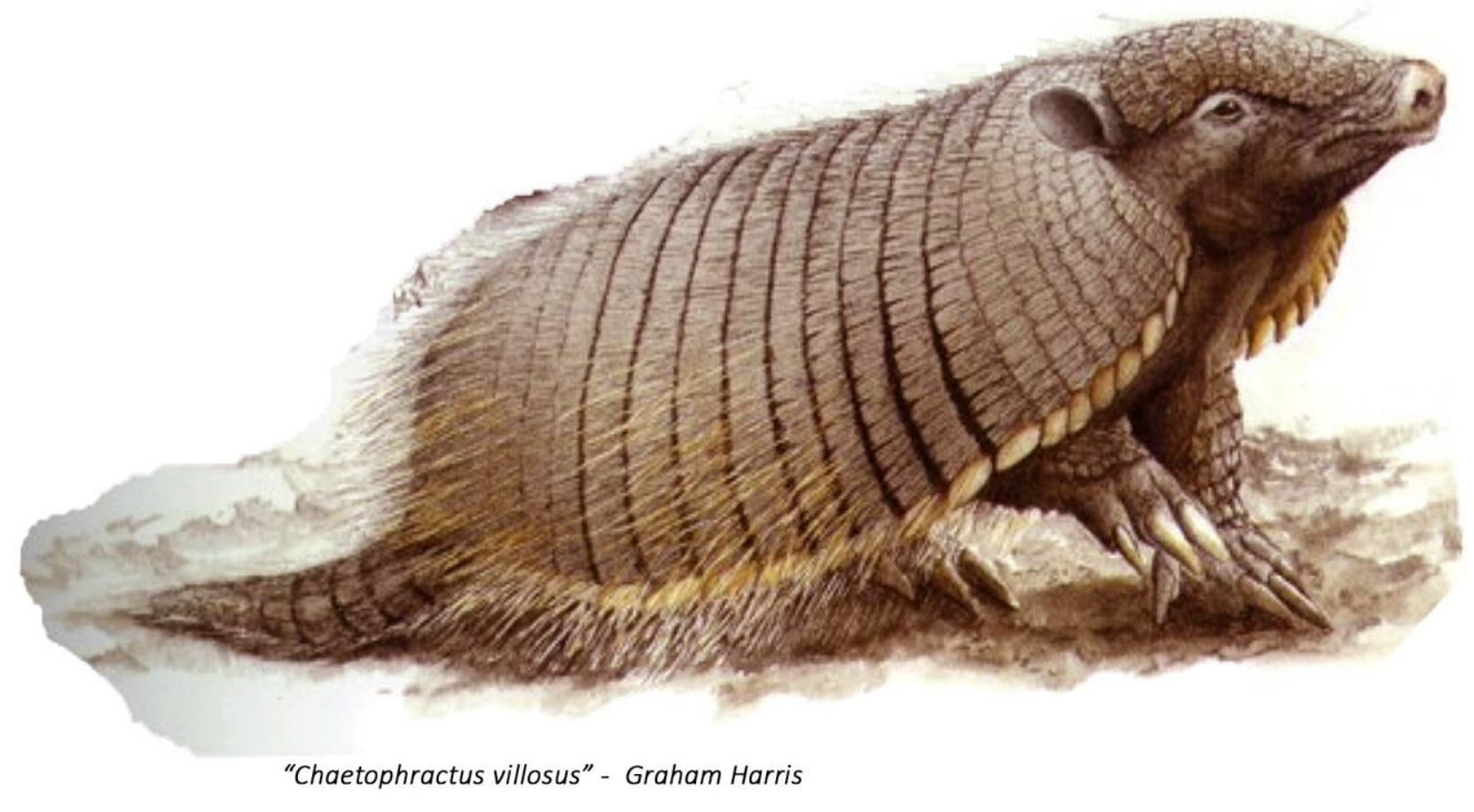

"Al trote como el peludo, monte espinudo suelo cruzar, no importa que sea duro, no tengo apuro para marchar..." 


\subsection{LAS INVASIONES BIOLÓGICAS COMO PROBLEMÁTICA ECOLÓGICA Y}

\section{SOCIAL}

Las especies invasoras se han convertido en un aspecto importante para la conservación de la biodiversidad (Vitousek et al., 1997). Una especie exótica invasora puede afectar la riqueza y abundancia de las especies nativas de un ecosistema a través de interacciones directas, como la depredación, o indirectas, alterando la disponibilidad de recursos, modificando la composición de las comunidades y/o alterando la dinámica de enfermedades de las especies nativas (D’Antonio and Dudley, 1995; Lymbery et al., 2014; Pyšek et al., 2020). Al momento de definirla, una invasión biológica es entendida como el establecimiento y dispersión de una especie exótica fuera de su rango de distribución natural (IUCN, 2000).

Para que una especie sea considerada invasora debe atravesar una secuencia de transiciones que le permitan sobrepasar las diferentes barreras de dispersión y establecerse fuera de su rango de distribución nativo (Figura 1.1; Goodwin et al., 1999; Kolar and Lodge, 2001; Blackburn et al., 2011; Pyšek et al., 2020). Desde el momento de la introducción, la especie exótica interactúa con el ecosistema invadido (Figura 1.1). Esta interacción determina si la especie introducida se logrará establecer y dispersar en el nuevo ambiente. Una especie exótica se convierte en invasora solo sí logra dispersar fuera

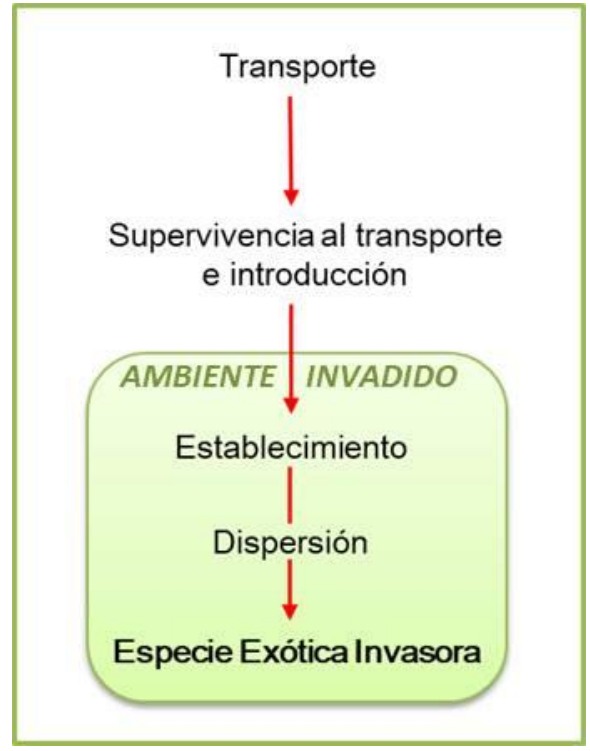

Figura 1.1 Transiciones que debe atravesar una especie introducida para convertirse en invasora (Modificado de Kolar and Lodge, 2001). 
del punto de introducción (Blackburn et al., 2011).

Mundialmente, la cantidad de especies introducidas se incrementa cada año y el número de especies que se convierten en invasoras tiene efectos ecológicos, económicos y sociales muy grandes (Richardson et al., 2011; Ballari et al., 2016; Pyšek et al., 2020). Y particularmente en ecosistemas insulares la introducción de vertebrados es una de las principales causas de alteración de las comunidades nativas (Russell et al., 2017). Mamíferos depredadores introducidos en islas, como ratas (Rattus spp.), mustélidos (Neovison vison) y gatos domésticos (Felis catus) han provocado la disminución de especies endémicas de roedores, reptiles y un gran número de colonias de aves (Courchamp et al., 2003; Blackburn et al., 2004). Por otro lado, especies omnívoras generalistas como jabalíes (Sus scrofa), suelen depredar nidos de aves y numerosas especies nativas (Cruz et al., 2005).

Por otro lado, los parásitos presentes en una especie introducida pueden tener una gran importancia sobre la ecología de los hospedadores, afectando directamente sobre la salud y el crecimiento de la población hospedadora y provocando nuevas infecciones sobre especies nativas (Hudson et al., 1998; Marcogliese, 2004), por lo tanto, no deberían ser pasados por alto al momento de hablar de una invasión biológica. Cuando una especie parasita sobrevive al proceso de introducción y permanece en la población hospedadora introducida, nos encontramos con un caso de "co-introducción parásitohospedador"(Lymbery et al., 2014). Por lo general cuando una especie introducida logra establecerse, lo hace con una diversidad y número de parásitos baja, lo cual puede significar una ventaja sobre las especies nativas, beneficiando el proceso de dispersión de la especie invasora (Torchin y Mitchell, 2004). Inclusive el hospedador introducido puede adquirir especies parásitas provenientes de hospedadores nativos, y amplificar o disminuir el riesgo de infección de los hospedadores nativos (Poulin, 2017).

Entender el proceso de invasión de una especie introducida incluye tanto aspectos ecológicos como sociales, debido a que el ser humano está involucrado en todo el proceso. La gran parte de las introducciones (accidentales o intencionales) tienen al ser humano como el principal agente de dispersión o vector. Así mismo, una vez que la 
especie se establece en determinada región, la percepción de la sociedad local acerca de la misma tendrá repercusiones en las decisiones que las autoridades pertinentes deban tomar para el manejo de la especie (García-llorente et al., 2008; Borgnia et al., 2013). Sin embargo, a pesar de que en la mayoría de los casos la introducción de especies se traduce en diversas problemáticas ecológicas, muchas veces una especie invasora puede resultar beneficiosa para la sociedad local y brindar algunos servicios ecositémicos. Por ejemplo, muchas especies son introducidas con fines ornamentales, recreativos o como agentes de control biológico. Inclusive muchas veces son parte de diversas prácticas culturales y económicas para muchas sociedades (Ewel et al., 1999; Schlaepfer et al., 2011). Por ello, al momento de estudiar cualquier invasión biológica es necesario contemplar tanto aspectos ecológicos como sociales, y así lograr un manejo integral de la especie introducida.

\subsection{CHAETOPHRACTUS VILLOSUS DENTRO DEL MAGNORDEN}

\section{XENARTHRA}

El magnorden Xenarthra Cope, 1889 , es un clado monofilético endémico de Sudamérica y constituye uno de los cuatro grandes grupos de mamíferos placentarios (Meredith et al., 2011; Gibb et al., 2016). Actualmente se encuentra representado por perezosos (Orden Pilosa Flower, 1883, Suborden Phyllophaga Owen, 1842), osos hormigueros (Orden Pilosa Flower, 1883, Suborden Vermilingua Illiger, 1811) y armadillos (Orden Cingulata Illiger, 1811). Los armadillos son los únicos mamíferos que poseen una

coraza formada por osteodermos cubiertos por escamas epidérmicas. Además sus extremidades anteriores son robustas y bien adaptadas a los hábitos semifosoriales.

La especie de armadillo que representa el objeto de estudio de esta tesis es Chaetophractus villosus, comúnmente conocido como el "peludo":

Familia Chlamyphoridae Gibb et al., 2016

Subfamilia Euphractinae Winge, 1923

Tribu Euphractini Winge, 1923

Género Chaetophractus Fitzinger, 1871

Chaetophractus villosus Desmarest, 1804 
El peludo es el segundo armadillo de mayor tamaño dentro de la subfamilia Euphractinae, llega a pesar entre $2 \mathrm{~kg}$ y $5 \mathrm{~kg}$. Es uno de los armadillos más ampliamente distribuidos en Argentina. Se lo encuentra en el Gran Chaco de Bolivia, Paraguay y gran parte de Argentina incluyendo Patagonia (Figura 1.2; Abba et al., 2012).

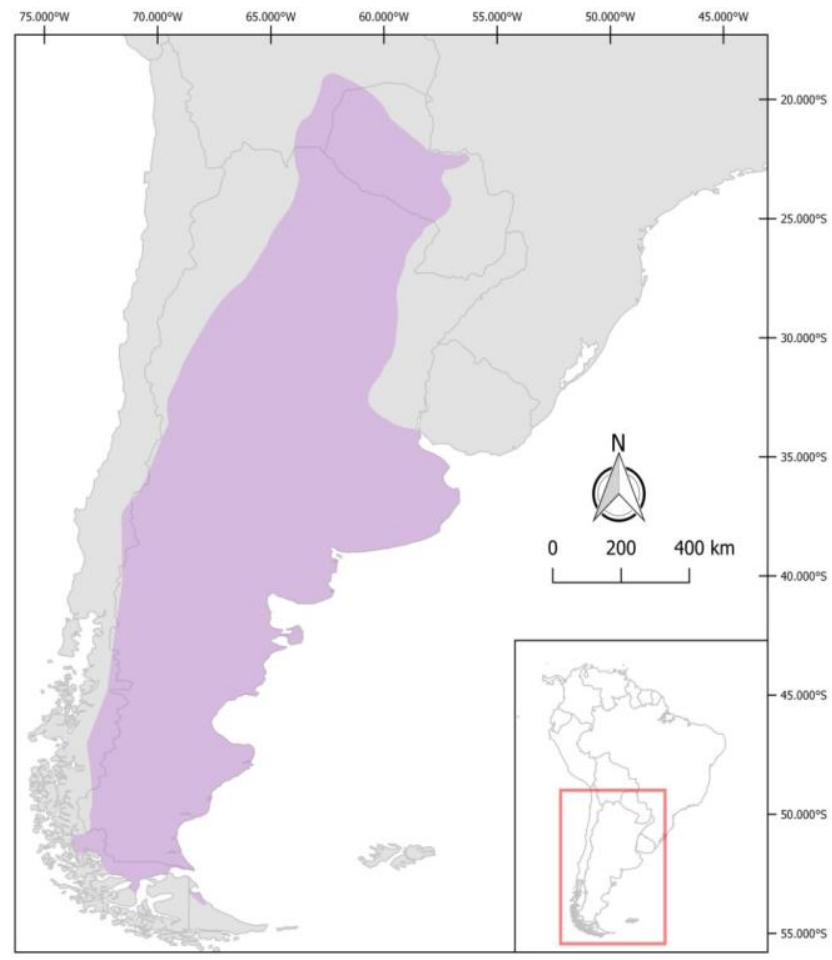

Figura 1.2. Distribución de Chaetophractus villosus. (Modificado de https://www.iucnredlist.org/)

El peludo es de hábitos nocturnos/crepusculares y se lo encuentra en diversos ambientes como pastizales, bosque, estepa e inclusive en agroecosistemas y otros ambientes degradados (Abba et al., 2012, 2015). Es considerado omnívoro-carnívoro. Se alimenta principalmente de invertebrados y vertebrados (Figura 1.3, Redford, 1985; Abba et al., 2010; Arriagada et al., 2017; Gallo et al., 2019a). Si bien la carroña (restos de animales en descomposición) es un ítem importante de la dieta de este armadillo, Gallo et al. (2019a) encontraron anfibios y reptiles completos al analizar contenidos estomacales, como así también cáscaras de huevo, lo que indica que este armadillo depreda activamente en ciertas ocasiones. Resulta común encontrar peludos intentando alimentarse sobre crías de ganado recién nacidas. Este comportamiento se ha visto en 
otros armadillos como Euphractus sexcinctus (Bezerra, 2001; Foster et al., 2017) y Dasypus novemcinctus (Nesbitt et al., 1977; Staller et al., 2005; Rader et al., 2007). Además, como todos los armadillos, C. villosus tiene hábitos semifosoriales y construye estructuras complejas para refugio (cuevas) y simples para alimentarse ("hozaduras"). El tipo de suelo es determinante al momento de construir estas estructuras. C. villosus prefiere suelos arenosos o calcáreos, evitando aquellos más rocosos (Abba and Vizcaíno, 2011; Ciuccio, 2014).

Si bien el peludo es una de las dos especies de armadillos que habitan Patagonia continental, el registro fósil y análisis filogeográficos sugieren que la población núcleo del peludo se originó en la región Pampeana (ver Poljak et al. 2010), siendo el Río Chubut el límite sur de su distribución. Su presencia en Patagonia centro-sur fue reportada hace aproximadamente 100 años (Abba et al., 2014). La dispersión de C. villosus a lo largo de Patagonia se vio favorecida por diversas actividades antrópicas, como ser el incremento de la producción agrícola y ganadera, lo que permitió un aumento de materia orgánica y macroinvertebrados en el suelo; la construcción de puentes y caminos, posibilitando a este armadillo superar barreras naturales como cuerpos de agua (insuperable para cualquier armadillo Euphractinae); y el avance de la industria petrolera, donde la construcción de oleoductos y gasoductos permitieron al peludo sobrellevar condiciones climáticas adversas, ya que el gas suele calentarse para ser transportado (Abba et al., 2014).

\section{3 ÁREA DE ESTUDIO: LA ISLA GRANDE DE TIERRA DEL FUEGO}

El archipiélago de Tierra del Fuego se ubica en la porción subantártica de sudamérica $\left(52^{\circ} \mathrm{S}-56^{\circ} \mathrm{O}\right.$, Figura 1.4$)$ y es considerado una de las últimas regiones silvestres en el planeta debido a sus vastas áreas de ambiente prístino y baja densidad poblacional (Mittermeier et al., 2002). Sin embargo, esta región aloja actualmente un gran número de especies introducidas (Silva and Saavedra, 2008; Valenzuela et al., 2013). La isla de mayor tamaño del archipiélago es la Isla Grande $\left(48.000 \mathrm{~km}^{2}\right)$. Esta isla puede ser 
dividida en tres grandes ambientes según su fisonomía. La estepa, el ecotono con bosque deciduo y el bosque subantártico (Figura 1.4).

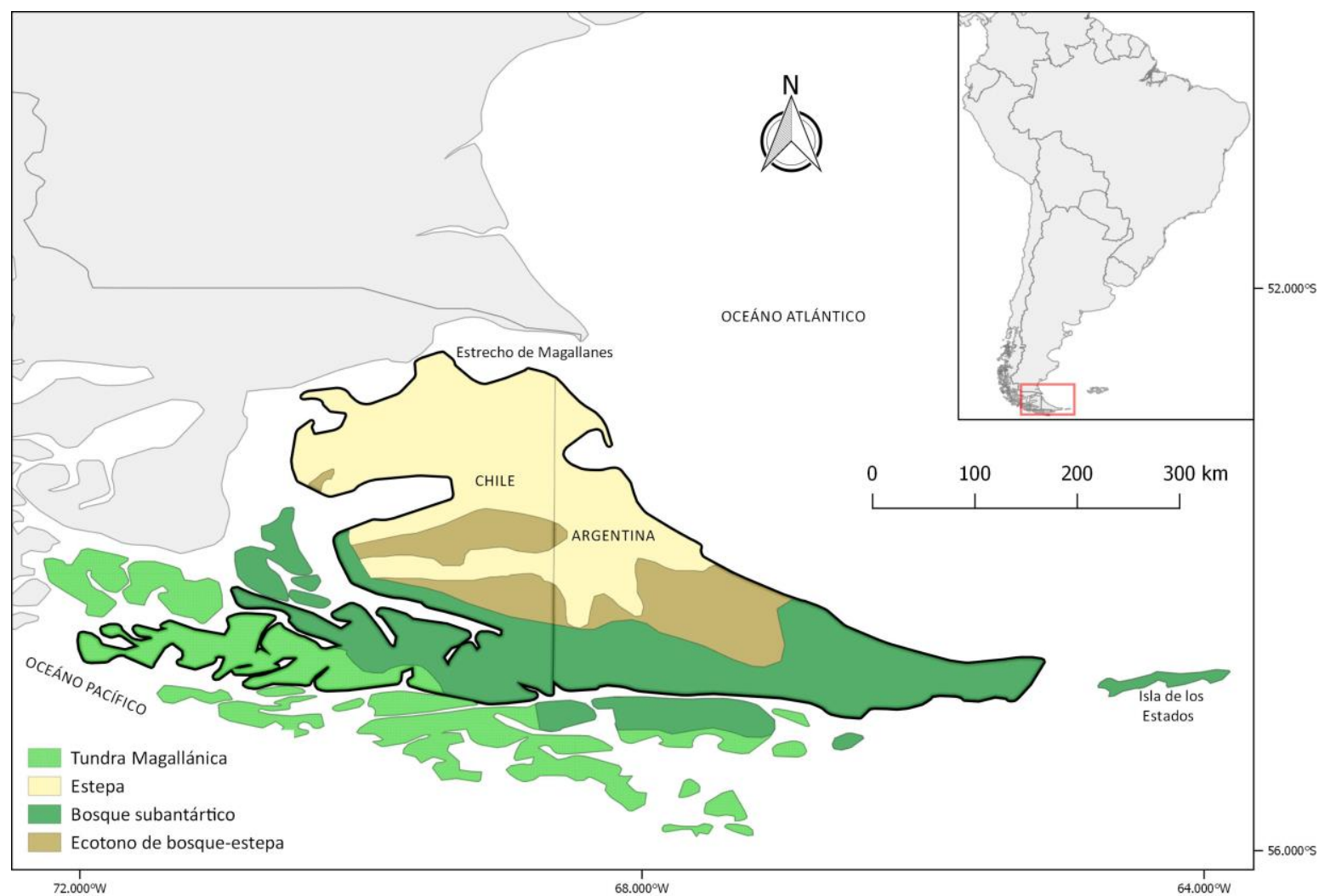

Figura 1.4. Ambientes del archipiélago fueguino. Con borde oscuro se destaca la Isla Grande de Tierra del Fuego. Modificado de Borromei et al. (2014)

La estepa magallánica es un mosaico complejo con gran diversidad (Figura 1.5; Collantes et al. 1999), se trata de un pastizal arbustivo, ubicado al noreste de la isla, dominada principalmente por Festuca gracillina (Coirón) y arbustos de Chilliotricum diffusum (Mata negra). En asociación con estas especies, y formando parches de menor densidad, se encuentran arbustos de Mata verde (Lepidophyllum cupressiforme), arbustos de Calafate (Barberis buxifolia), Murtilla (Empetrum rubrum) y diferentes especies de herbáceas (Tuhkanen et al., 1990; Collantes et al., 1999). A pesar de la gran diversidad florística de la estepa, muchas áreas de la isla se encuentran altamente degradadas por el 
sobrepastoreo del ganado ovino (Collantes et al., 2013) y actividades asociadas a la extracción de petróleo y gas.

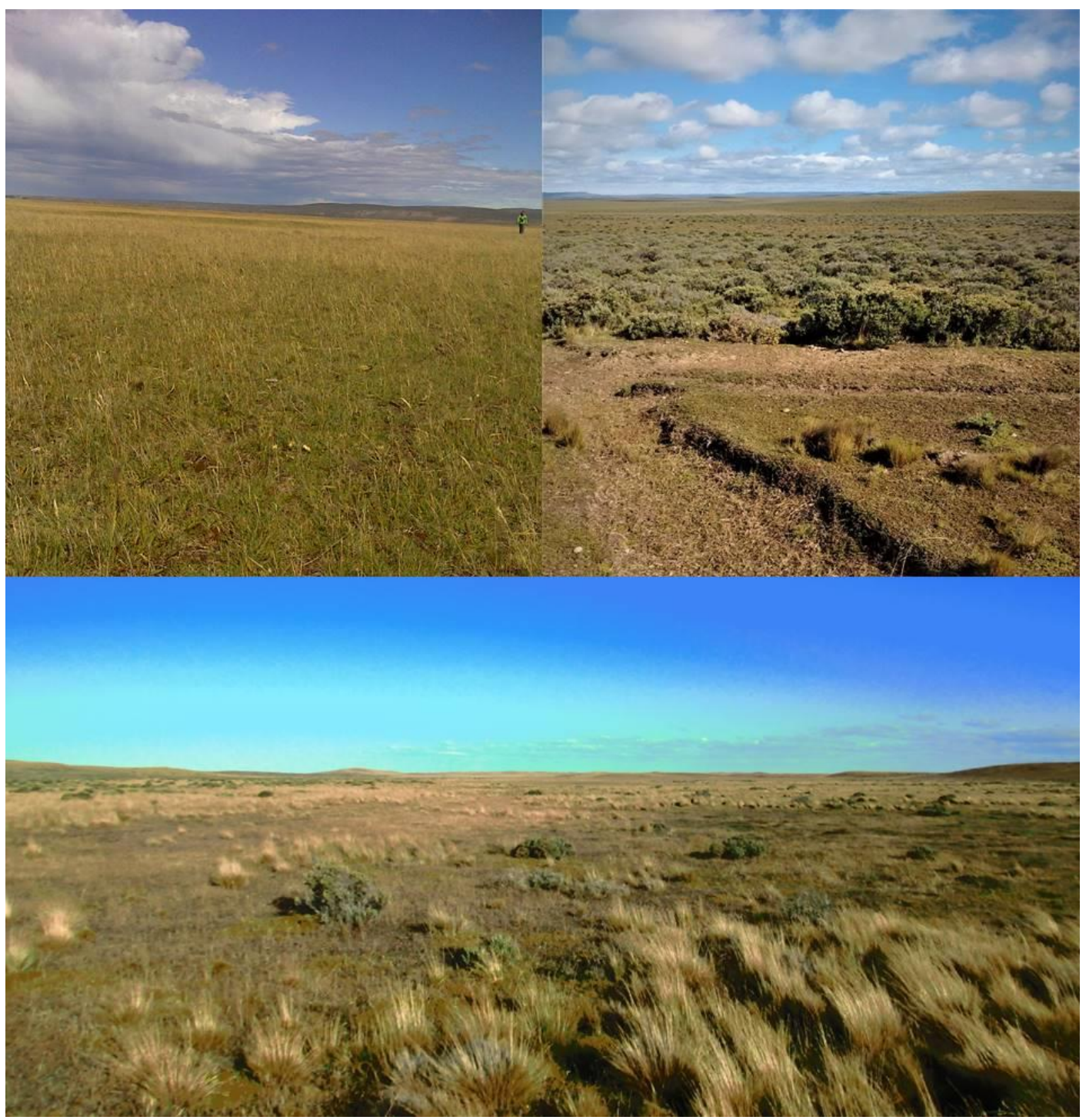

Figura 1.5. Diversidad en la fisonomía de la estepa Magallánica

En la porción central de la isla, al sur del Río Grande, se encuentra una región discontinua de Ñires (Nothofagus antartica), que forma un ecotono entre el bosque continuo (Figura 1.6) y la estepa. La porción más austral de la isla está dominada por el bosque subantartico, en el cual se hallan dos especies de Nothophagus. El suelo rocoso y la presencia de turberas (Moore, 1983) hacen que este ambiente sea inhabitable para los armadillos (Abba and Vizcaíno, 2011; Ciuccio, 2014), los cuales dependen exclusivamente 
del tipo de suelo para poder construir sus madrigueras. Por lo tanto en esta tesis se concentraron los esfuerzos de muestreo en la estepa y el ecotono, es decir en la mitad norte de la isla.

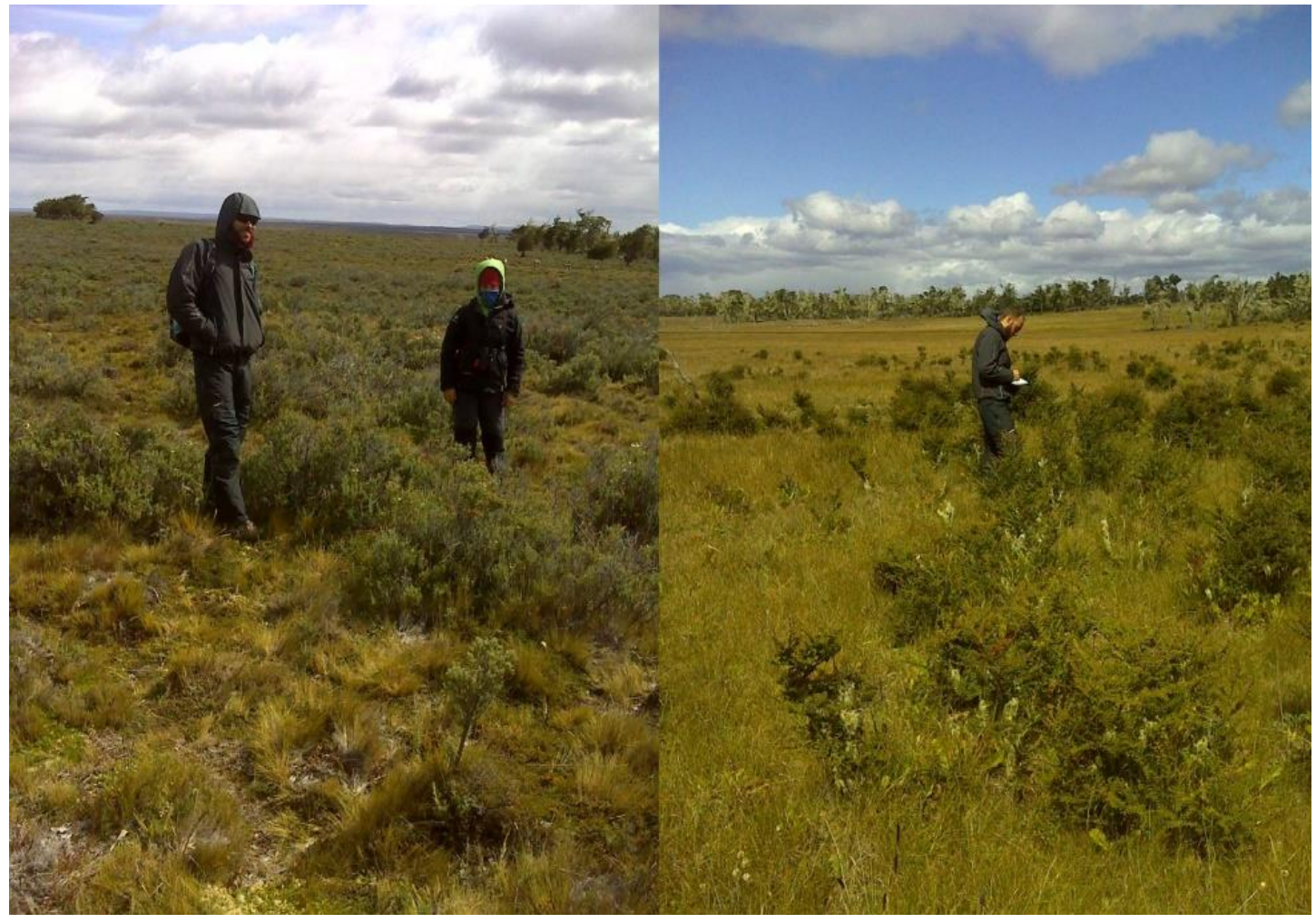

Figura 1.6. Ecotono con el bosque subantartico

La temperatura media en la estepa y el ecotono, se ubica desde los $-3^{\circ} \mathrm{C}$ a $0^{\circ} \mathrm{C}$ en invierno y de $\operatorname{los} 11^{\circ} \mathrm{C}$ a $16,3^{\circ} \mathrm{C}$ durante el verano. La precipitación anual es de 200 a 400 mm (datos tomados de la Estación Astronómica de la ciudad de Río Grande, EARG-UNLPCONICET).

\subsection{ChAETOPHRACTUS VILLOSUS EN TIERRA DEL FUEGO: POSIBLES} IMPACTOS Y ANTECEDENTES DE ARMADILLOS INVASORES

Deferrari et al. (2002) reportaron, en un resumen presentado en las VII Jornadas Argentinas de Mastozoología, la presencia de un nuevo mamífero introducido en la Isla 
Grande de Tierra del Fuego, el peludo. Cinco años después Poljak et al. (2007) publicaron el primer trabajo sobre este armadillo exótico. Los autores describen que el peludo fue introducido en la isla a mediados de la década de 1980 y que $C$. villosus logró adaptarse al adverso clima fueguino al asociarse con las redes de gasoductos presentes en la parte norte de la isla. Debido a que los armadillos, al igual que todos los xenartros, son homeotermos imperfectos (poseen una capacidad limitada para regular su temperatura corporal), estas líneas de tuberías, que poseen una temperatura constante de aproximadamente $4^{\circ} \mathrm{C}$ para facilitar el transporte de gas, habrían favorecido el establecimiento del peludo en la región (Poljak et al., 2007).

El caso del peludo en Tierra del Fuego no es el primero en cuanto a armadillos invasores. En América del Norte, la mulita de 9 bandas (Dasypus novemcinctus) invadió la región centro-sur de los Estados Unidos. Originalmente, el límite norte de la distribución de $D$. novemcinctus se encontraba en el Río Bravo (frontera entre México y EEUU), el cual funcionaba como barrera natural. A principios de la década de 1850 , se reportó la presencia de armadillos en Texas, Estados Unidos. Posteriormente su rango se fue expandiendo hacia el noreste del país, probablemente facilitado por los colonos europeos presentes en la zona (Taulman and Robbins, 1996). El aumento del comercio y las nuevas prácticas agrícolas favorecieron la traslocación y la dispersión de la mulita. Sin embargo, durante el siglo XX, la invasión continuó por dos vías diferentes. La expansión desde Texas continuó en todas direcciones y paralelamente ocurrieron varias liberaciones de individuos en cautiverio en la región central del estado de Florida, llegando a ocupar todo el estado.

Si bien los armadillos no son conocidos por sus comportamientos depredatorios, es sabido que pueden alimentarse de huevos de aves y reptiles y pequeños vertebrados (Dalponte and Tavares-Filho, 2004; McDonough et al., 2007; Rader et al., 2007). Particularmente el peludo incluye en su dieta huevos de aves que nidifican en el suelo y pequeños roedores (Arriagada et al., 2017; Gallo et al., 2019). Inclusive hay registros de peludos atacando individuos de Pingüinos y Cauquenes (Frere et al., 1992; Abba et al., 2010b; Cossa N., datos no publicados). La Isla Grande de Tierra del Fuego forma parte del 
área de reproducción para varias aves que anidan en el suelo como ser los cauquenes, dentro de los cuales encontramos al cauquén colorado (Chloephaga rubidiceps) que se encuentra en Peligro Crítico de extinción para Argentina (AA/AOP and SAyDS 2008, Cossa et al., 2017). De esta manera Chaetophractus villosus se sumaría al ensamble de mamíferos invasores presentes en la isla, como el visón americano (Neovison vison) y el zorro gris (Lycalopex gymnocercus), perjudicando aún más la situación poblacional de estas aves. A su vez, la Isla Grande de Tierra del fuego es el hábitat de dos pequeños vertebrados endémicos de la región, el tuco-tuco magallánico (Ctenomys magellanicus) y la lagartija austral (Liolaemus magellanicus), por lo que la presencia de un nuevo mamífero invasor en la región podría perjudicar a especies nativas.

\subsection{OBJETIVOS E HIPÓTESIS}

El objetivo general de esta tesis es actualizar la distribución del peludo (Chaetophractus villosus) en Tierra del Fuego, interpretar los procesos que facilitaron su expansión, analizar la dinámica de los parásitos de este mamífero recientemente introducido en la isla y hacer una evaluación de los impactos potenciales sobre especies nativas. Este objetivo general da marco a los siguientes objetivos específicos, los cuales permiten comprender el proceso de invasión de este mamífero en la Isla Grande de Tierra del Fuego:

A. Determinar la expansión alcanzado por el peludo (Chaetophractus villosus) en Tierra del Fuego desde los muestreos publicados 13 años atrás (ver Poljak et al., 2007).

B. Analizar las asociaciones con características del ambiente (e.g. vegetación dominante, tipo de suelo) y la distancia a estructuras del paisaje (e.g. tubería de hidrocarburos) que podrían permitir al peludo dispersar en una región de clima tan extremo como el existente en Tierra del Fuego (e.g. cambio en el tipo de dieta, cambio de comportamiento, etc.).

C. Conocer la percepción social de la población de Tierra del Fuego sobre la presencia del peludo. 
D. Describir y analizar las variaciones en la helmintofauna de las dos especies de armadillos de Patagonia (C. villosus y Zaedyus pichiy) respecto a localidades al norte del Río Chubut.

E. Evaluar si el peludo, un mamífero mediano y omnívoro, causa algún impacto sobre especies autóctonas (e.g. aves que nidifican en el suelo).

F. Elaborar una serie de recomendaciones para el desarrollo de un plan de control y monitoreo de Chaetophractus villosus para la Isla Grande de Tierra del Fuego.

A partir de los objetivos antes mencionados, se ponen a prueba las siguientes hipótesis:

- Chaetophractus villosus está colonizando nuevas áreas en Tierra del Fuego, facilitado por la actividad del hombre.

- La población invasora de Chaetophractus villosus en Tierra del Fuego incorporó, en los últimos 10 años, parásitos presentes en la fauna fueguina.

- Chaetophractus villosus afecta a la fauna autóctona a través de la depredación de aves que nidifican en el suelo.

- La sociedad local percibe a este armadillo como perjudicial, debido principalmente a las cuevas que construyen o a sus hábitos alimenticios.

\section{BIBLIOGRAFÍA}

AbBa, A. M., M. J. NabTe, ANd D. E. U. SAUthier. 2010. New data on armadillos (Xenarthra: Dasypodidae) for central Patagonia, Argentina. Edentata 11:11-17.

Abba, A. M., S. Poljak, M. Gabrielli, P. Teta, And U. F. J. Pardiñas. 2014. Armored invaders in Patagonia: recent southward dispersion of armadillos (CINGULATA, DASYPODIDAE. Mastozoología neotropical 21:311-318.

Abba, A. M., M. F. Tognelli, V. P. Seitz, J. B. Bender, and S. F. Vizcaíno. 2012. Distribution of extant xenarthrans (Mammalia: Xenarthra) in Argentina using species distribution 
models. Mammalia 76:123-136.

AbBA, A. M., AND S. F. VizCAíno. 2011. Distribución de los armadillos (Xenarthra: Dasypodidae) en la provincia de Buenos Aires, Argentina. Mastozoología Neotropical 18:185-206.

AbBA, A. M., E. Zufiaurre, M. Codesido, AND D. N. BilencA. 2015. Burrowing activity by armadillos in agroecosystems of central Argentina: Biogeography, land use, and rainfall effects. Agriculture, Ecosystems and Environment 200:54-61.

ARRIAgAdA, A. ET AL. 2017. Hábitos alimenticios de poblaciones periféricas de Zaedyus pichiy y Chaetophractus villosus (Cingulata, Chlamyphoridae) en la Patagonia chilena. Iheringia. Série Zoologia 107:1-8.

Ballari, S. A., C. B. Anderson, And A. E. J. Valenzuela. 2016. Understanding trends in biological invasions by introduced mammals in southern South America: A review of research and management. Mammal Review 46:229-240.

BezerRA, A. M. R. 2001. Predation of rodents by the Yellow Armadillo (Euphractus sexcinctus) in Cerrado of the Central Brazil. Mammalia 65:86-88.

BLACKBURN, T. M. ET AL. 2011. A proposed unified framework for biological invasions. Trends in Ecology and Evolution 26:333-339.

Blackburn, T. M., P. CAssey, R. P. Duncan, K. L. Evans, and K. J. Gaston. 2004. Avian extinction and mammalian introductions on oceanic islands. Science 305:1955-1958.

BorgniA, M., V. BeNITEZ, C. GozZI, Y M. L. GUICHÓN. 2013. La ardilla de vientre rojo en Argentina y el manejo de especies introducidas como un problema biológico y social. Ecologia Austral 23:147-155.

Borromei A. M., J. F. Ponce, A. Coronato, M. S. Candel, D. Olivera and M. Okuda. 2014. Reconstrucción de la vegetación posglacial y su relación con el ascenso relativo del nivel del mar en el extremo este del canal beagle, Tierra del Fuego, Argentina. Andean Geology 41:362-379.

CıUccıo, M. 2014. Ecología comportamental de los dasipódidos en el pastizal pampeano, con particular consideración de los hábitos alimenticios. Enfoque eco-morfofisiológico. Universidad Nacional del Sur. 
Collantes, M., J. Anchorena, And A. Cingolani. 1999. The steppes of Tierra del Fuego:

Floristic and growthform patterns controlled by soil fertility and moisture Article. Plant Ecology 140:41-42.

Collantes, M. B., C. Escartín, K. Braun, A. Cingolanl, and J. Anchorena. 2013. Grazing and grazing exclusion along a resource gradient in magellanic meadows of Tierra del Fuego. Rangeland and Ecology Managment 66:688-699.

Cossa, N. A., L. Fasola, I. Roesler, And J. C. Reboreda. 2017. Ruddy-headed Goose Chloephaga rubidiceps: former plague and present protected species on the edge of extinction. Bird Conservation International 27:269-281.

COURChAMP, F., J.-L. ChAPUIS, AND M. PASCAL. 2003. Mammal invaders on islands: impact, control and control impact. Biological Reviews 78:347-383.

Cruz, F., C. Josh donlan, K. Campbell, ANd V. Carrion. 2005. Conservation action in the Galàpagos: Feral pig (Sus scrofa) eradication from Santiago Island. Biological Conservation 121:473-478.

D’ANTONIO, C. M., AND T. L. DudLEY. 1995. Biological invasions as agents of change on islands versus mainlands. Pp. 103-121 in Islands, Ecological Studies (Analysis and Synthesis) (A. H. Vitousek P.M., Loope L.L., ed.). Springer, Berlin.

Dalponte, J. C., AND J. A. TAVARES-FILHo. 2004. Diet of the Yellow Armadillo, Euphractus sexcinctus, in South-Central Brazil. Edentata 6:37-41.

Deferrari, G., Camilion, C., Escobar, J., y Lizarralde, M. S. 2002. Presencia de Chaetophractus villosus en Tierra del Fuego: nueva especie introducida. ¿Nuevo problema?. Libro de Resumenes VII Jornadas Argentinas de Mastozoología. Mendoza, Argentina

EWEL, J. J. ET AL. 1999. Deliberate Introductions of Species: Research Needs. BioScience 49:619-630.

Foster, V. C., G. Porfirio, D. Viana, P. SARmento, And E. Fischer. 2017. Yellow armadillos (Euphractus sexcinctus) can predate on vertebrates as large as a chicken. Mammalia 81:319-322.

Frere, E., P. Gandini, And P. Dee Boersma. 1992. Effects of nest type and location on reproductive success of the Magellanic penguin Spheniscus magallanicus. Marine 
Ornithology. .

Gallo, J., L. FAsola, AND A. ABBA. 2019. Armadillos as pest controllers? Food habits of five armadillos in Argentina. Mastozoologia Neotropical.

García-llorente, M., B. Martín-López, J. A. González, And C. Montes. 2008. Social perceptions of the impacts and benefits of invasive alien species: implications for management. Biological Conservation 141:2969-2983.

GIBB, G. C. ET AL. 2016. Shotgun mitogenomics provides a reference phylogenetic framework and timescale for living xenarthrans. Molecular Biology and Evolution 33:621-642.

GoOdWIN, B. J., A. J. MCALLISTER, AND L. FAHRIG. 1999. Predicting invasiveness of plant species based on biological information. Conservation Biology 13:422-426.

Hudson, P. J., A. P. Dobson, AND D. NEWBORN. 1998. Prevention of population cycles by parasite removal. Science 282:2256-2258.

IUCN. 2000. Guidelines for the prevention of biodiversity loss caused by alien species. Gland, Switzerland.

KOLAR, C. S., AND D. M. LODGE. 2001. Progress in invasion biology: predicting invaders. Trends in Ecology and Evolution 16:199--204.

Lymbery, A. J., M. Morine, H. G. Kanani, S. J. Beatty, ANd D. L. Morgan. 2014. Co-invaders: The effects of alien parasites on native hosts. International Journal for Parasitology: Parasites and Wildlife 3:171-177.

MARCOGLIESE, D. J. 2004. Parasites: Small players with crucial roles in the ecological theater. EcoHealth 1:151-264.

Mcdonough, C. M., J. M. LockHART, AND W. J. Loughry. 2007. Population dynamics of ninebanded armadillos: insights from a removal experiment. Southeastern Naturalist $6: 381-392$

MEREDITH, R. W. ET AL. 2011. Impacts of the cretaceous terrestrial revolution and KPg extinction on mammal diversification. Science 334:521-524.

Mittermeier, R. A. M., C. G. Pilgrim, J. Fonseca, G. Konstant, and R. William. 2002.

Wilderness: Earth's last wild places. CEMEX, México. 
Moore, D. M. 1983. Flora of Tierra del Fuego. Anthony Nelson Ltd., Shrewsbury, Shropshire, U.K.

Nesbitt, S. A., W. M. Hetrick, L. E. Williams, and D. A. Austin. 1977. Foods of the nine-banded armadillo in Florida. Proceedings of the Annual Conference of the Southeastern Association of Fish \& Wildlife Agencies. .

Poljak, S., V. Confalonieri, M. Fasanella, M. Gabrielli, and M. S. Lizarralde. 2010. Phylogeography of the armadillo Chaetophractus villosus (Dasypodidae Xenarthra): Post-glacial range expansion from Pampas to Patagonia (Argentina). Molecular Phylogenetics and Evolution 55:38-46.

Poljak, S., J. Escobar, G. Deferrari, ANd M. LizArRalde. 2007. Un nuevo mamífero introducido en la Tierra del Fuego: El "peludo" Chaetophractus villosus (Mammalia, Dasypodidae) en Isla Grande. Revista Chilena de Historia Natural 80:285-294.

Poulin, R. 2017. Invasion ecology meets parasitology: Advances and challenges. International Journal for Parasitology: Parasites and Wildlife:6-8.

PYŠEK, P. ET AL. 2020. Scientists' warning on invasive alien species. Biological Reviews.

Rader, M. J., T. W. Teinert, L. A. Brennan, F. Hernández, N. J. Silvy, And X. Ben Wu. 2007. Identifying predators and nest fates of bobwhites in southern Texas. Journal of Wildlife Management 71:1626-1630.

REDFORD, K. H. 1985. Food habits of armadillos (XENARTHRA: DASYPODIDAE). Pp. 429-437 in The evolution and ecology of armadillos, sloths and vermilinguas (G. . Montgomery, ed.). Smithsonian Institution Press, Washington \& London.

RICHARDSON, D. M., P. PYSEK, AND C. J.T. 2011. A compendium of essential concepts and terminology in biological invasions. Pp. 409-420 in Fifty Years of Invasion Ecology: The Legacy of Charles Elton (D. M. Richardson, ed.). Blackwell Publishing, Oxford.

RusselL, J. C., J. Y. MeYer, N. D. Holmes, AND S. PAGAD. 2017. Invasive alien species on islands: Impacts, distribution, interactions and management. Environmental Conservation 44:359-370.

SChlaEpfer, M. A., D. F. SAX, AND J. D. Olden. 2011. The potential conservation value of nonnative species. Conservation Biology 25:428-437. 
Silva, C. A, AND B. SAAVEDRA. 2008. Knowing for controlling: ecological effects of invasive vertebrates in Tierral del Fuego. Revista Chilena de Historia Natural 81:123-136.

Staller, E. L., J. P. Carroll, R. P. Thornton, W. E. Palmer, and D. C. Sisson. 2005. Identifying predators at northern bobwhite nests. Journal of Wildlife Management 69:124-132.

TAULMAN, J. F., AND L. W. RoBBINS. 1996. Recent range expansion and distributional limits of the nine-banded armadillo (Dasypus novemcinctus) in the United States. Journal of Biogeography 23:635-648.

TORChin, M. E., AND C. E. MitChell. 2004. Parasites, pathogens, and invasions by plants and animals. Frontiers in Ecology and the Environment 2:183-190.

Tuhkanen, S., I. KuokKa, S. Hyvönen, S. Stenroos, And J. Niemelä. 1990. Tierra del Fuego as a target for biogeographical research in the past and present. Anales del Instituto de la Patagonia 19:4-107.

Valenzuela, A. E. J., A. Raya Rey, L. Fasola, And A. SChiavini. 2013. Understanding the interspecific dynamics of two co-existing predators in the Tierra del Fuego Archipelago: The native southern river otter and the exotic American mink. Biological Invasions 15:645-656.

Vitousek, P. M., C. M. D’antonio, L. L. Lloyd, M. RejMÁnek, And R. Westbrooks. 1997. Introduced species: a significant component of human-caused global change. New Zeland Journal of Ecology 21:1-16. 


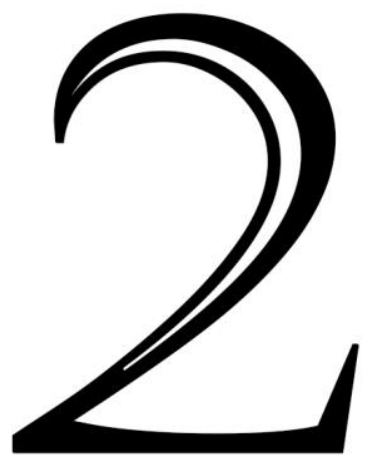

\section{DISTRIBUCIÓN Y USO DEL ESPACIO DEL PELUDO EN TIERRA DEL FUEGO}

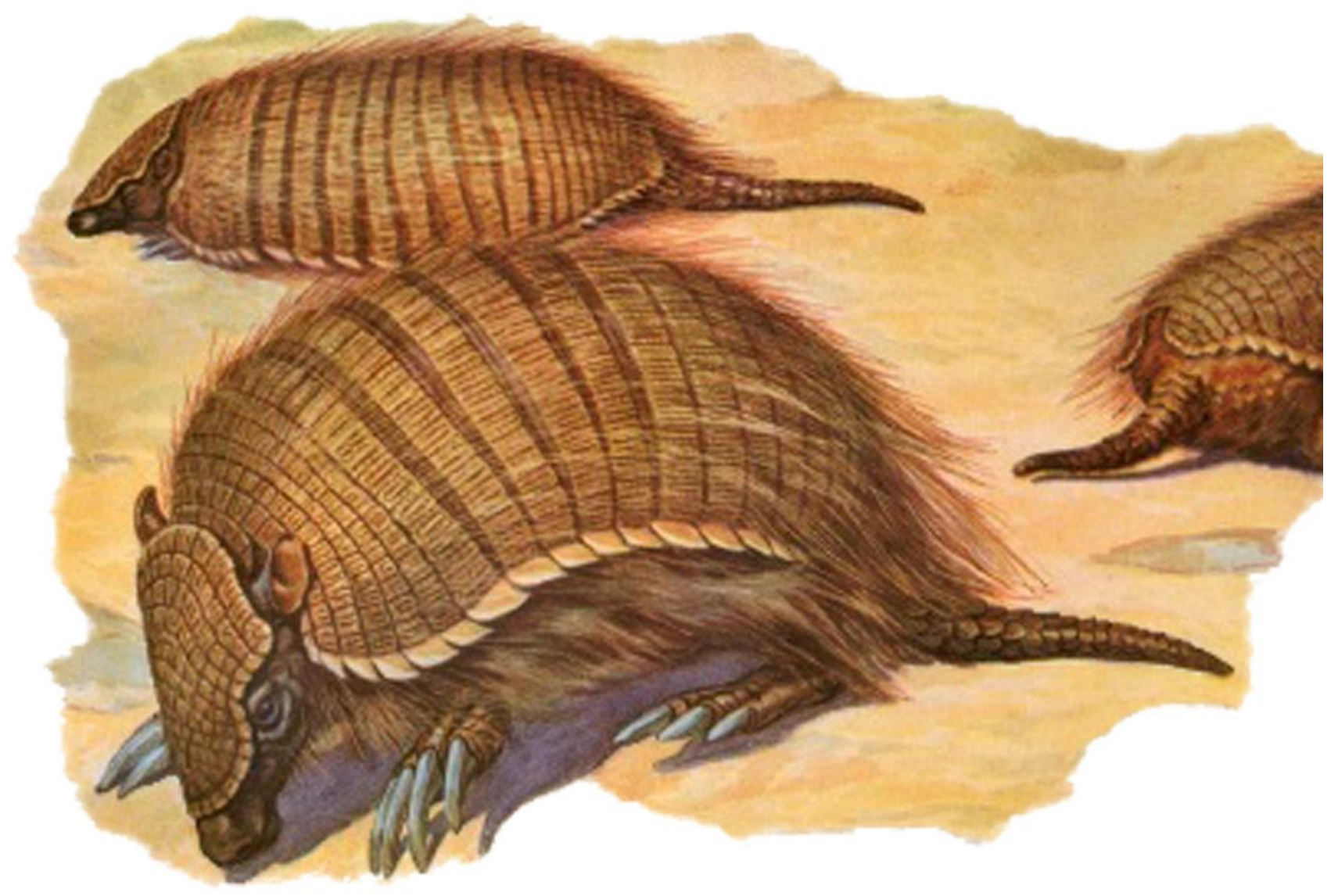

"Peludos" - Dibujo de Carlos Wiedner

Cabrera A \& Yepes J-1960 - Mamíferos Sud-Americanos.

"Hijo ai pucha quirquincho, Que te has metido en la cueva, La cabeza en el aujero, y la colita pa'juera!" 


\subsection{INTRODUCCIÓN}

El peludo (Chaetophractus villosus) pertenece a uno de los 9 géneros y 14 especies de armadillos presentes en Argentina (Teta et al., 2018). Actualmente su distribución es la más amplia en relación con el resto de las especies, no obstante el registro fósil y datos filogreográficos aseguran que su origen fue en la región pampeana (Poljak et al., 2010), y que su dispersión hacia Patagonia es muy reciente (ocurrió hace aproximadamente 100 años, Abba et al., 2014). A lo largo de todo su rango de distribución, el peludo habita una gran variedad de hábitats incluyendo pastizales, bosques, estepa e inclusive áreas de producción agrícola-ganaderas (Abba et al., 2012, 2015; Gallo et al., 2019b). Esta especie es activa durante todo el año y su reproducción se concentra en primavera-verano (ver Gallo et al. 2019b). Sus hábitos alimenticios son variados e incluyen invertebrados y vertebrados como roedores, lagartijas e incluso aves que nidifican en el suelo (Abba et al., 2010; Arriagada et al., 2017; Gallo et al., 2019a).

Al igual que todos los armadillos, el peludo tiene hábitos semi-fosoriales y construye dos tipos de estructuras en el suelo. Por un lado estructuras complejas a modo de cuevas que utilizan como refugio y por otro, estructuras más simples conocidas como hozaduras, utilizadas para conseguir alimento (Abba et al., 2005). La construcción de estas cuevas como refugio está estrechamente asociada a la baja tasa metabólica que poseen los xenartros en general (McNab, 1980). Al ser homeotermos imperfectos, las cuevas subterráneas le permiten regular mejor su temperatura corporal (Grassé, 1955; Boily, 2002). En su distribución nativa, particularmente en la región pampeana, Abba et al. (2005, 2007a) encontraron mayor cantidad de cuevas en zonas de pastizales naturales con suelo húmico y en terrenos elevados. A su vez, en estudios posteriores se encontró mayor actividad de $C$. villosus en suelos arenosos y con buena permeabilidad, debido a la facilidad para cavar cuevas y hozaduras (see Abba et al., 2015; Gallo et al., 2019b).

La introducción de $C$. villosus en la Isla Grande de Tierra del Fuego ocurrió hace aproximadamente 35 años, cuando 8 individuos fueron liberados al norte de la ciudad de Río Grande aparentemente por razones estéticas (Figura 2.1; ver Capítulo 3; Poljak et al., 2007). La región Norte de la Isla es un área de alta actividad petrolera, además de la 
tradicional actividad ganadera. Actualmente cuenta con 6 plantas de tratamientos de hidrocarburos y más $300 \mathrm{~km}$ de gasoductos y oleoductos, los cuales transportan petróleo y gas hacia el sur de la isla y al continente (Figura 2.1).

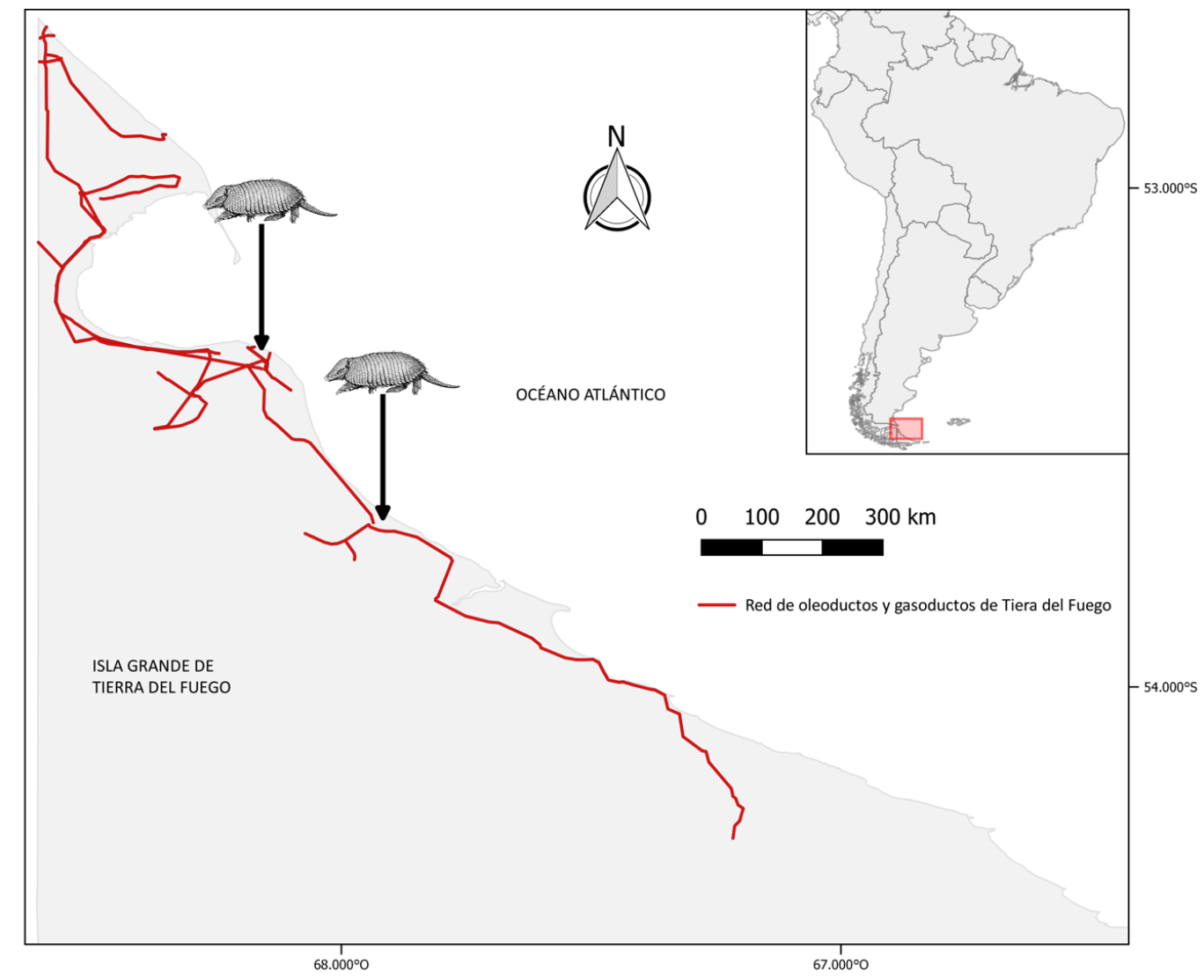

Figura 2.1. Puntos de introducción de individuos de $C$. villosus (flechas) y principales gasoductos y oleoductos.

El primer trabajo sobre esta especie introducida fue publicado por Poljak et al.

(2007). Estos autores reportaron que la distribución de este armadillo en la isla abarcaba una superficie de $482 \mathrm{~km}^{2}$ asociada estrechamente a la red de gasoductos (Figura 2.2). En estas tuberías, el gas es calentado a una temperatura promedio de $4^{\circ} \mathrm{C}$ para facilitar su transporte. Dada las condiciones ambientales, la baja tasa metabólica y la alta sensibilidad a cambios en la temperatura ambiente que poseen los armadillos, la temperatura del suelo cercano a los gasoductos habría favorecido el establecimiento de $C$. villosus en la isla (Poljak et al., 2007). 


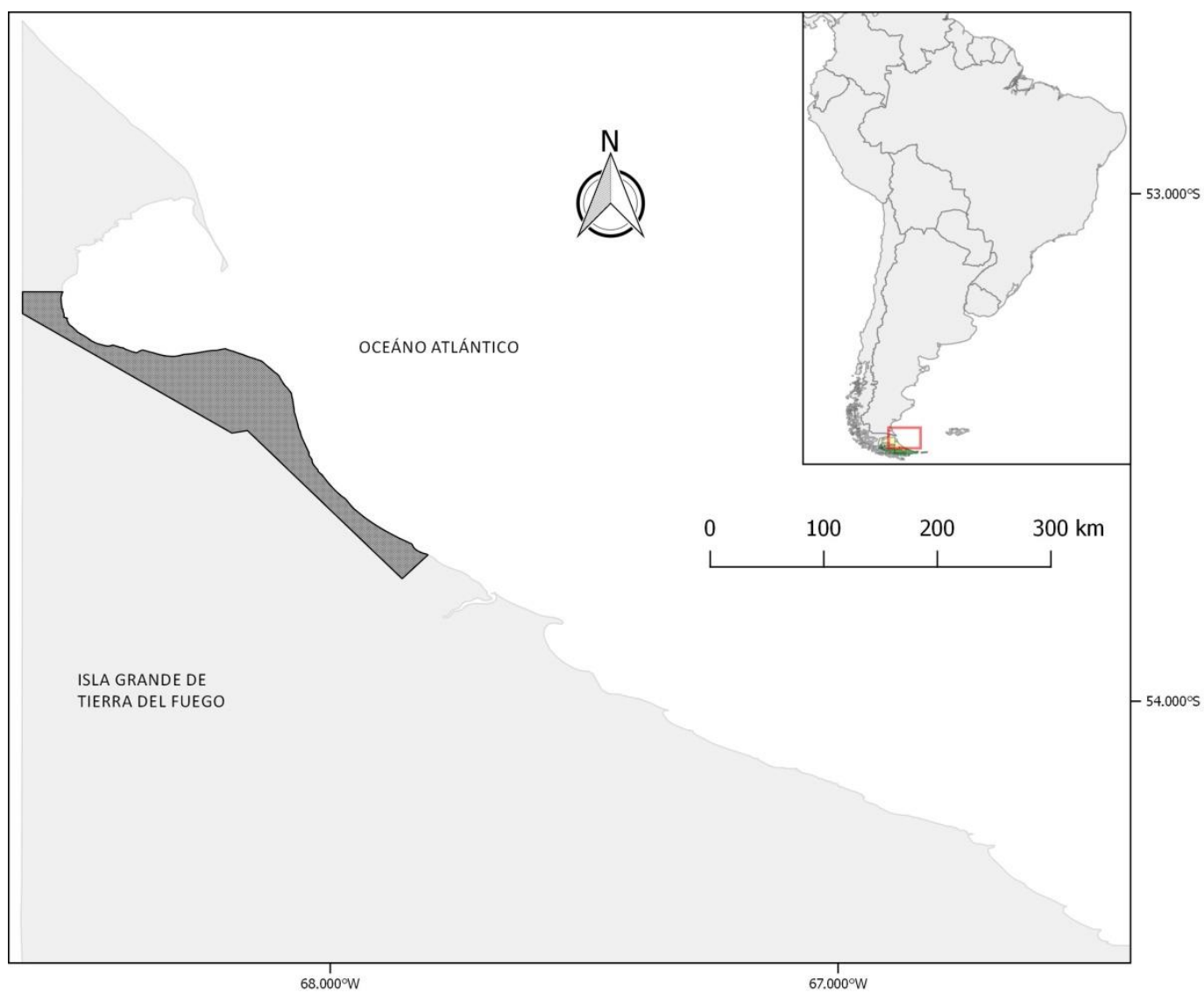

Figura 2.2. Distribución de Chaetophractus villosus estimada por Poljak et

al. (2007)

El último estudio realizado fue en base a muestreos iniciados en 2002 y finalizados en 2006 (ver Poljak et al., 2007), y a partir de lo allí postulado y el tiempo transcurrido desde dicho estudio, surgieron las siguientes preguntas:

(1) ¿Alguna característica del ambiente fueguino favorece la presencia y expansión del peludo en la Isla Grande de Tierra del Fuego?

(2)¿La distribución actual del peludo sigue asociada a los gasoductos de la Isla Grande de Tierra del Fuego como postuló Poljak et al. (2007)?

El objetivo de este capítulo es actualizar la distribución del peludo en la Isla Grande de Tierra del Fuego, evaluar las asociaciones entre algunas características del ambiente con la presencia, el uso del espacio por parte del peludo y para comprender que ha favorecido su expansión por la Isla. 


\subsection{MATERIALES Y MÉTODOS}

\subsection{1 Área de estudio y diseño experimental}

Durante la temporada de verano de 2017 y 2018 se realizaron dos campañas de muestreo en la Isla Grande de Tierra del Fuego. El área de estudio abarcó la estepa y el ecotono fueguino, ya que en el bosque subantártico el suelo rocoso y la presencia de turberas (Moore, 1983) hacen que este ambiente sea inhabitable por los armadillos (Abba and Vizcaíno, 2011; Ciuccio, 2014), los cuales dependen exclusivamente del tipo de suelo para poder construir sus cuevas (Ver Capítulo 1). A partir de una grilla de celdas cuadradas de $25 \mathrm{~m}^{2}$ se seleccionaron al azar 52 celdas (Figura 2.3), las cuales representan el $38 \%$ del total de celdas que interceptaron caminos principales o secundarios, este número se

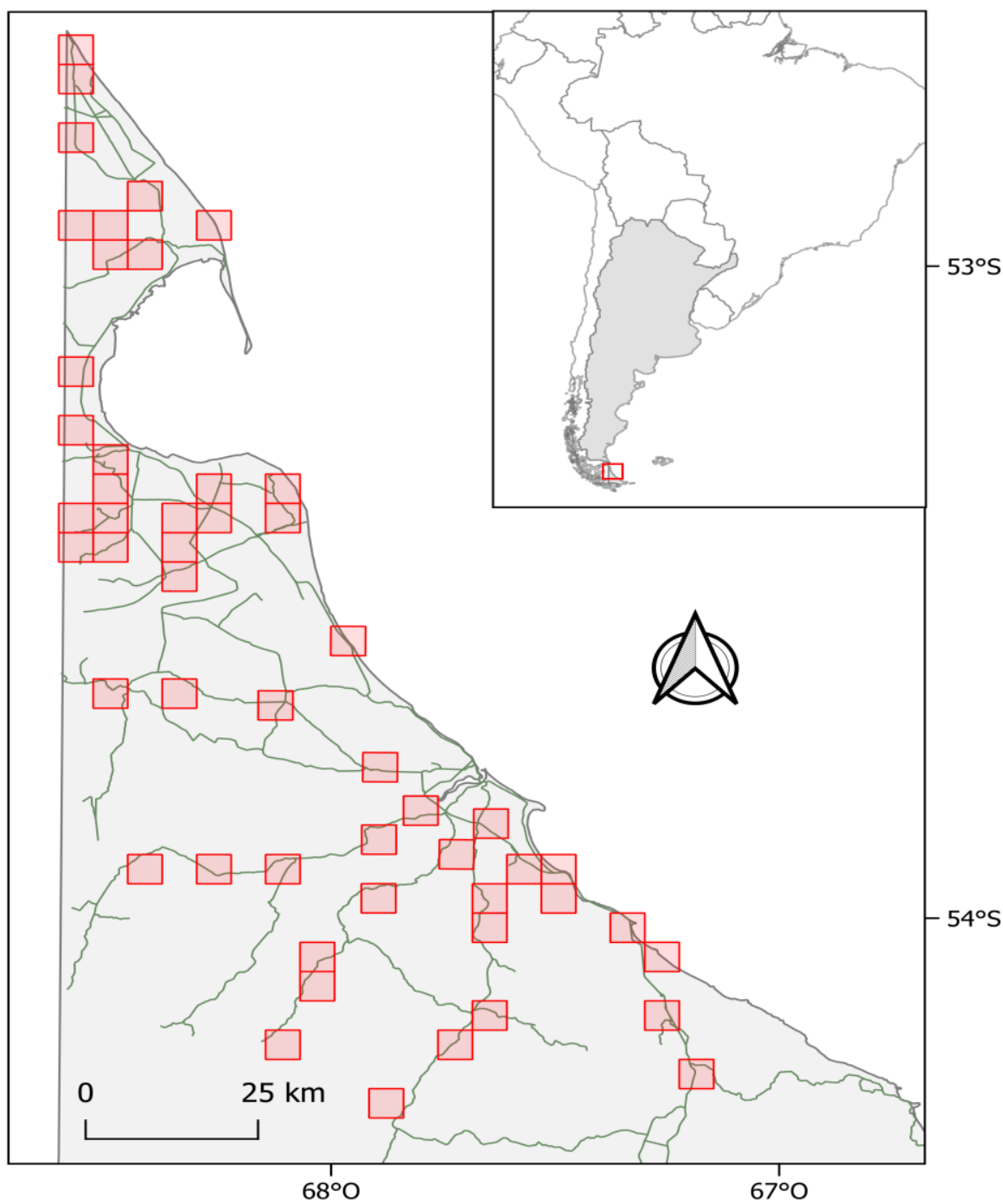

Figura 2.3. Celdas y caminos donde se llevó a cabo el muestreo de $C$. villosus 
ajustó a las posibilidades logísticas disponibles logrando un número que fuera suficiente para aplicar las pruebas estadísticas.

Treinta y cinco celdas seleccionadas correspondieron a la estepa magallánica y 17 al ecotono. La cantidad de celdas seleccionadas en cada ambiente es proporcional a la disponibilidad de cada ambiente y a la presencia de caminos. En cada celda se ubicó una transecta de $600 \mathrm{~m}$ de largo por $60 \mathrm{~m}$ de ancho, cuyo punto de inicio fue a los $30 \mathrm{~m}$ del camino. En cada transecta, tres personas separadas por $20 \mathrm{~m}$ recorrieron una línea (paralela con las otras) y registraron la presencia (sí/no) y la abundancia (número) de evidencias indirectas de presencia de armadillos: cuevas y hozaduras (Abba et al., 2005).

\subsubsection{Ocurrencia y Uso del espacio}

Estudios sobre la especie en otras regiones de Argentina identificaron asociaciones a las características del terreno y de la vegetación (Abba et al., 2007; 2014) . Sin embargo, nuevos ambientes pueden plantear diferentes asociaciones que permitan a las especies colonizarlos. En cada transecta se registró el tipo de vegetación de acuerdo a la especie dominante: Coirón (Festuca gracillina), Mata negra (Chilliotricum diffusum), Mata verde (Lepidophyllum cupressiforme), Herbaceas (áreas solo con especies de hierbas), Murtilla (Empetrum rubrum) y Ñires (Nothofagus antarctica) para explorar asociaciones con especies vegetales dominantes en particular. Cada transecta fue luego caracterizada según:

1) Características de la vegetación: se consideró la altura dominante de la vegetación y el nivel de degradación de la misma debido al pastoreo como variables que reflejen la estructura de la vegetación y condición. La altura de la vegetación fue dividida en tres clases: vegetación alta $(>1 \mathrm{~m})$, vegetación media $(0,2 m-1 m)$ y vegetación baja $(<0,2 m)$. Para definir el nivel de degradación nos basamos en el impacto del ganado ovino sobre la vegetación principalmente (sobrepastoreo), de esta manera distinguimos visualmente entre degradado y no degradado. 
2) Características del terreno: se consideró el tipo de suelo dominante y la elevación del terreno como variables limitantes para la construcción de cuevas para el peludo. Los tipos de suelo fueron clasificados como: Húmico (suelos con alto contenido de humus), arenoso, húmico-arenoso, arcilloso y pedregosoarenoso. Debido a su baja frecuencia de aparición los tipos arcilloso y pedregosoarenoso fueron combinados en la categoría "otros" para el análisis de datos. La topografía, o elevación del terreno, fue clasificada en: terreno elevado (colinas, bordes de caminos), terreno bajo o llano y terreno inundable.

Considerando las variables antes mencionadas, se realizaron los siguientes análisis:

1. Análisis de ocurrencia: se evaluaron las asociaciones de diferentes características del ambiente local (a nivel de transecta) con la presencia de armadillos. Se realizó una prueba de independencia de chi-cuadrado para explorar las asociaciones entre la presencia/ausencia de arma dillos y la identidad de las especies vegetales dominantes (el tipo de vegetación). Luego, se usaron Modelos Lineales Generalizados con una distribución de error de Bernoulli para evaluar las asociaciones entre la presencia de la especie con las características del terreno y con las características de la vegetación.

2. Análisis de intensidad de uso del espacio: se analizó la asociación entre las características del terreno y entre las características de la vegetación con el número de evidencias indirectas encontradas. En este caso se usaron Modelos Lineales Generalizados con una distribución del error binomial negativa.

Los análisis se hicieron para las características de la vegetación (altura y degradación) por un lado y para las características del terreno (suelo y elevación) por otro, ya que en estudios anteriores en el área de distribución nativa (Abba et al., 2007; 2014) se determinaron asociaciones con el suelo y con la vegetación. De esta manera se pudo evaluar si estas asociaciones se mantenían o se modificaban en esta área recientemente colonizada.

Para todos los análisis se utilizó la plataforma R (R Core Team 2016). 


\subsubsection{Influencia de la distancia a estructuras del paisaje en la presencia de la}

\section{especie.}

Basándonos en estudios previos de $C$. villosus en la región continental de América del Sur (Abba et al., 2005, 2007, 2009, 2014, 2015, Rimoldi y Abba, 2013) y el trabajo previo realizado en la Isla Grande de Tierra del Fuego (Poljak et al., 2007), es posible que ciertas estructuras del paisaje sean beneficiosas para este armadillo. Estructuras naturales y artificiales como gasoductos, ríos, asentamientos humanos e inclusive la costa marina pueden proveer a estos armadillos de alimento y refugio. Para poner a prueba esta hipótesis, se analizó la asociación entre la presencia de armadillos y la distancia a ciertas estructuras del paisaje de la Isla Grande de Tierra del Fuego usando Modelos Lineales Generalizados con una distribución del error de Bernoulli. Las variables explicatorias para estos modelos fueron en todos los casos la distancia mínima entre la transecta con evidencias indirectas de peludos (ver sección 2.1) y cada una de las estructuras del paisaje nombradas. Tales distancias fueron extraídas de un SIG utilizando la herramienta NNJoin del software QGIS (QGIS Development Team, 2017).

\subsubsection{Tasa de expansión}

Tomando como situación inicial la distribución estimada por Poljak et al. (2007) (Figura 2.2) se calculó la Tasa de expansión lineal para los últimos 13 años, considerando como inicio al punto más extremo del límite sur estimado por los citados autores. El punto final fue situado en la transecta positiva más extrema encontrada en este estudio. Además se calculó el área de distribución actual usando el método del Mínimo Polígono Convexo, siguiendo la metodología de la UICN para establecer el "Área de Ocupación" de una especie (International Union for Conservation of Nature, 2001).

\subsection{5. Áreas de concentración de presencia e intensidad de uso}


Además se desarrolló una imagen raster (tamaño de pixel $432 \mathrm{~m} \times 432 \mathrm{~m}$ ) basada en la densidad de signos en cada transecta para visualizar mejor las zonas con mayor concentración de actividad de peludos y así poder identificar áreas para el manejo de la especie (control/erradicación). Para crear este mapa se usó la función “HeatMap” de QGIS.

\subsection{RESULTADOS}

Se encontraron evidencias indirectas de armadillos (cuevas y hozaduras) en 33 de las 52 transectas realizadas (62\%; Figura 2.4).

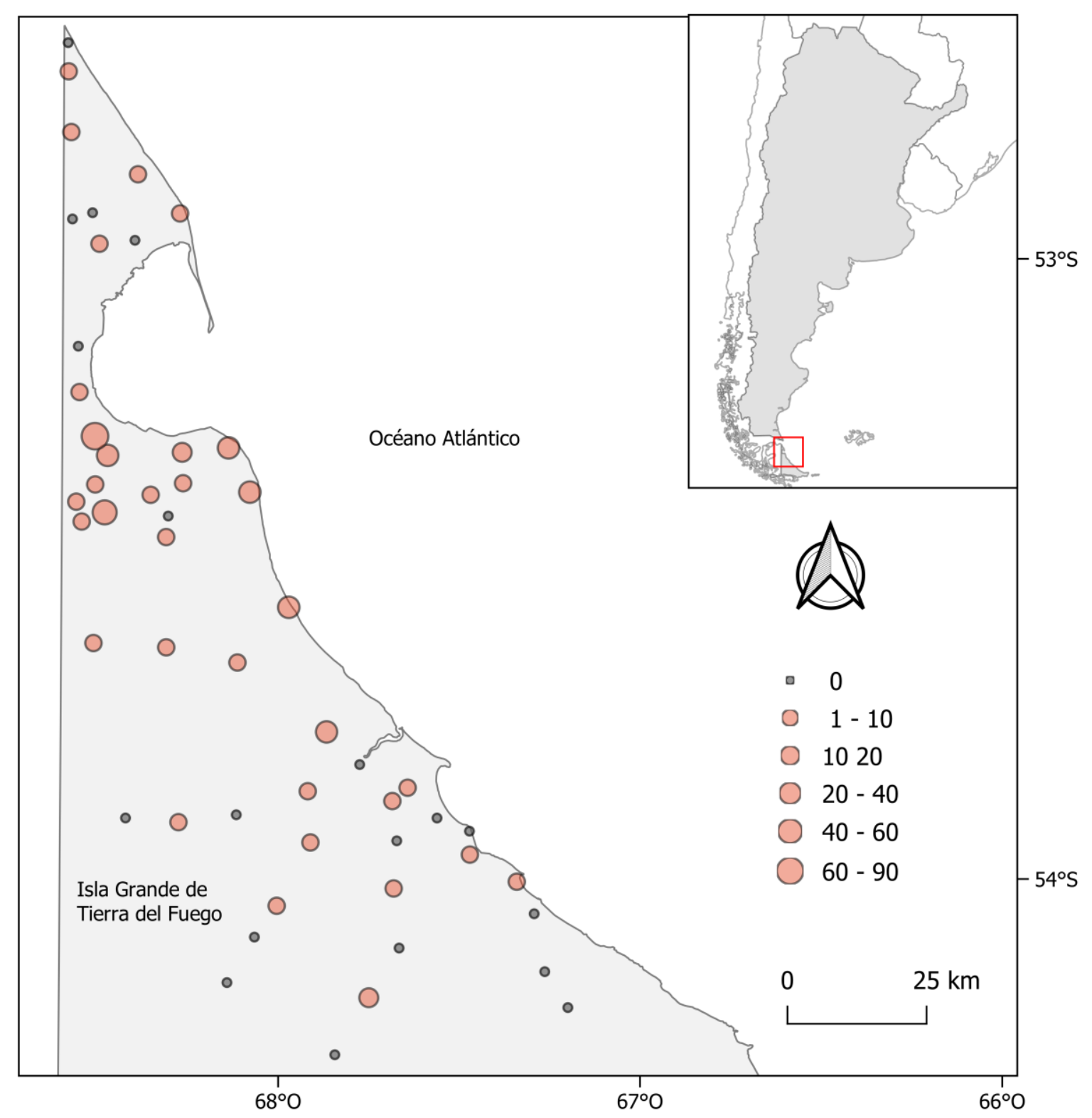

Figura 2.4. Frecuencia (intervalos) de evidencias indirectas de $C$. villosus en cada transecta. 
No se encontró asociación entre el tipo de vegetación y la presencia de armadillos $\left(\chi^{2}=2,32, p=0,675\right.$, d.f. $\left.=4\right)$.

Se pudo observar una asociación negativa a terrenos de baja elevación e inundables mientras que se encontró asociación positiva entre la presencia de armadillos y la altura de vegetación alta y media, como ser arbustos y Coirones respectivamente (Tabla 2.1).

Tabla 2.1. Resultados del análisis de asociación entre la presencia de la especie Chaetophractus villosus en las diferentes transectas y variables relacionadas al tipo de terreno y características de la vegetación. IC= Intervalo de Confianza del 95\%. Las variables explicatorias cuya asociación resultó significativa (IC no incluyen el 0 ) a la presencia de $C$. villosus están marcadas en negrita.

\begin{tabular}{|c|c|c|c|c|c|}
\hline \multirow[b]{2}{*}{ Variable analizada } & \multirow[b]{2}{*}{ Variable explicatoria } & \multirow[b]{2}{*}{ Estimador } & \multirow[b]{2}{*}{ SE } & \multicolumn{2}{|c|}{ IC } \\
\hline & & & & Inferior & Superior \\
\hline Presencia/ausencia de & Características del terreno & & & & \\
\hline \multirow[t]{11}{*}{ evidencias } & Intercepto* & 2,90 & 1,03 & 1,32 & 5,79 \\
\hline & Suelo Húmico & 2,43 & 1,48 & $-0,20$ & 6,04 \\
\hline & Suelo húmico-arenoso & $-2,95$ & 1,70 & $-6,85$ & 0,29 \\
\hline & Otros suelos & $-2,22$ & 1,59 & $-5,74$ & 1,12 \\
\hline & Terreno inundable & $-5,86$ & 1,80 & $-10,34$ & $-3,01$ \\
\hline & Terreno llano & $-4,73$ & 1,51 & $-8,46$ & $-2,23$ \\
\hline & Características de la vegetación & & & & \\
\hline & Intercepto** & $-1,19$ & 0,97 & $-3,17$ & 0,33 \\
\hline & Altura Media & 1,67 & 0,99 & 0,10 & 3,67 \\
\hline & Altura “Alta" & 2,58 & 1,60 & 0,09 & 5,93 \\
\hline & Degradada & 1,34 & 1,01 & $-0,24$ & 3,37 \\
\hline
\end{tabular}

${ }^{*}$ ) Intercepto es el valor que toma el modelo cuando el sitio corresponde a terrenos elevados y suelos arenosos $y\left({ }^{* *}\right)$ vegetación baja y no degradada.

En cuanto a la intensidad de uso del espacio por parte de $C$. villosus, el análisis mostró que la frecuencia de evidencias indirectas en una transecta estuvo asociada positiva con el suelo de tipo húmico (Tabla 2.2). Por otro lado, se encontró una asociación negativa con terrenos bajos e inundables y a la vegetación baja. 
Tabla 2.2. Resultados del análisis de asociación entre el $n^{\circ}$ de evidencias indirectas de Chaetophractus villosus en las diferentes transectas y variables relacionadas al tipo de terreno y características de la vegetación. Análisis de IC= Intervalo de Confianza del 95\%. Las variables explicatorias asociadas significativamente a una mayor frecuencia de evidencias de $C$. villosus están marcadas en negrita

\begin{tabular}{|c|c|c|c|c|c|}
\hline \multirow[b]{2}{*}{ Variable analizada } & \multirow[b]{2}{*}{ Variable explicatoria } & \multirow[b]{2}{*}{ Estimador } & \multirow[b]{2}{*}{ SE } & \multicolumn{2}{|c|}{ IC } \\
\hline & & & & Inferior & Superior \\
\hline \multirow[t]{12}{*}{ Número de evidencias } & Características del terreno & & & & \\
\hline & Intercepto* & 1,93 & 0,36 & 1,29 & 2,70 \\
\hline & Terreno inundable & $-1,74$ & 0,66 & $-3,38$ & $-0,16$ \\
\hline & Terreno llano & $-2,33$ & 0,72 & $-3,77$ & $-0,80$ \\
\hline & Suelo húmico & 1,77 & 0,58 & 0,40 & 3,44 \\
\hline & Suelo Húmico-arenoso & $-0,69$ & 0,87 & $-2,14$ & 1,11 \\
\hline & Otro suelo & $-1,57$ & 1,01 & $-3,37$ & 0,73 \\
\hline & Características de la vegetación & & & & \\
\hline & Intercepto** & 2,97 & 0,81 & 1,70 & 5,09 \\
\hline & Altura media & $-1,07$ & 0,89 & $-3,29$ & 0,46 \\
\hline & Altura baja & $-2,47$ & 1,05 & $-4,82$ & $-0,62$ \\
\hline & Degradada & 0,32 & 0,66 & $-0,82$ & 1,60 \\
\hline
\end{tabular}

$\left(^{*}\right)$ Intercepto es el valor que toma el modelo cuando el sitio corresponde a terrenos elevados y suelos arenosos y $\left(^{* *}\right)$ vegetación alta y no degradada.

No se encontró relación significativa entre la presencia de armadillos y la distancia a diferentes estructuras del paisaje naturales o antrópicas (Tabla 2.3).

Tabla 2.3. Análisis de la relación entre la presencia de Chaetophractus villosus y la distancia a diversas características del paisaje. IC= Intervalo de Confianza del $95 \%$.

IC

\begin{tabular}{lccccc} 
Variable analizada & Variable explicatoria & Estimador & SE & Inferior & Superior \\
\hline Presencia/Ausencia & Características del paisaje & & & & \\
de evidencias & Intercepto & $-5,4 \mathrm{E}-01$ & $5,8 \mathrm{E}-01$ & $-1,7 \mathrm{E}+00$ & $5,2 \mathrm{E}-01$ \\
& Asentamientos humanos & $2,3 \mathrm{E}-05$ & $5,4 \mathrm{E}-05$ & $-8,6 \mathrm{E}-05$ & $1,3 \mathrm{E}-04$ \\
& Ríos & $9,9 \mathrm{E}-06$ & $9,1 \mathrm{E}-05$ & $-1,9 \mathrm{E}-04$ & $1,7 \mathrm{E}-04$ \\
& Costa Marina & $-7,3 \mathrm{E}-06$ & $4,8 \mathrm{E}-05$ & $-1,1 \mathrm{E} 04$ & $8,5 \mathrm{E}-05$ \\
& Gasoductos/oleoductos & $-1,6 \mathrm{E}-06$ & $48 \mathrm{E}-05$ & $-9,2 \mathrm{E}-05$ & $9,8 \mathrm{E}-05$ \\
\hline
\end{tabular}


La tasa linear de expansión estimada fue de 10,9 km/año (131 km en 13 años). El método del Mínimo Polígono Convexo permitió calcular que el área (mínima) ocupada por C. villosus es de $5218 \mathrm{~km}^{2}$, de esta manera la distribución del peludo en la porción Argentina de la Isla Grande de Tierra del Fuego se incrementó 4736 km² desde 2007

(Figura 2.5b; Poljak et al., 2007).

El mapa de calor creado a partir de la densidad de evidencias indirectas (Figura

2.5a) permitió delimitar dos grandes áreas ( $\mathrm{A}$ y $B$ ) con alta frecuencia de uso por parte de los armadillos.

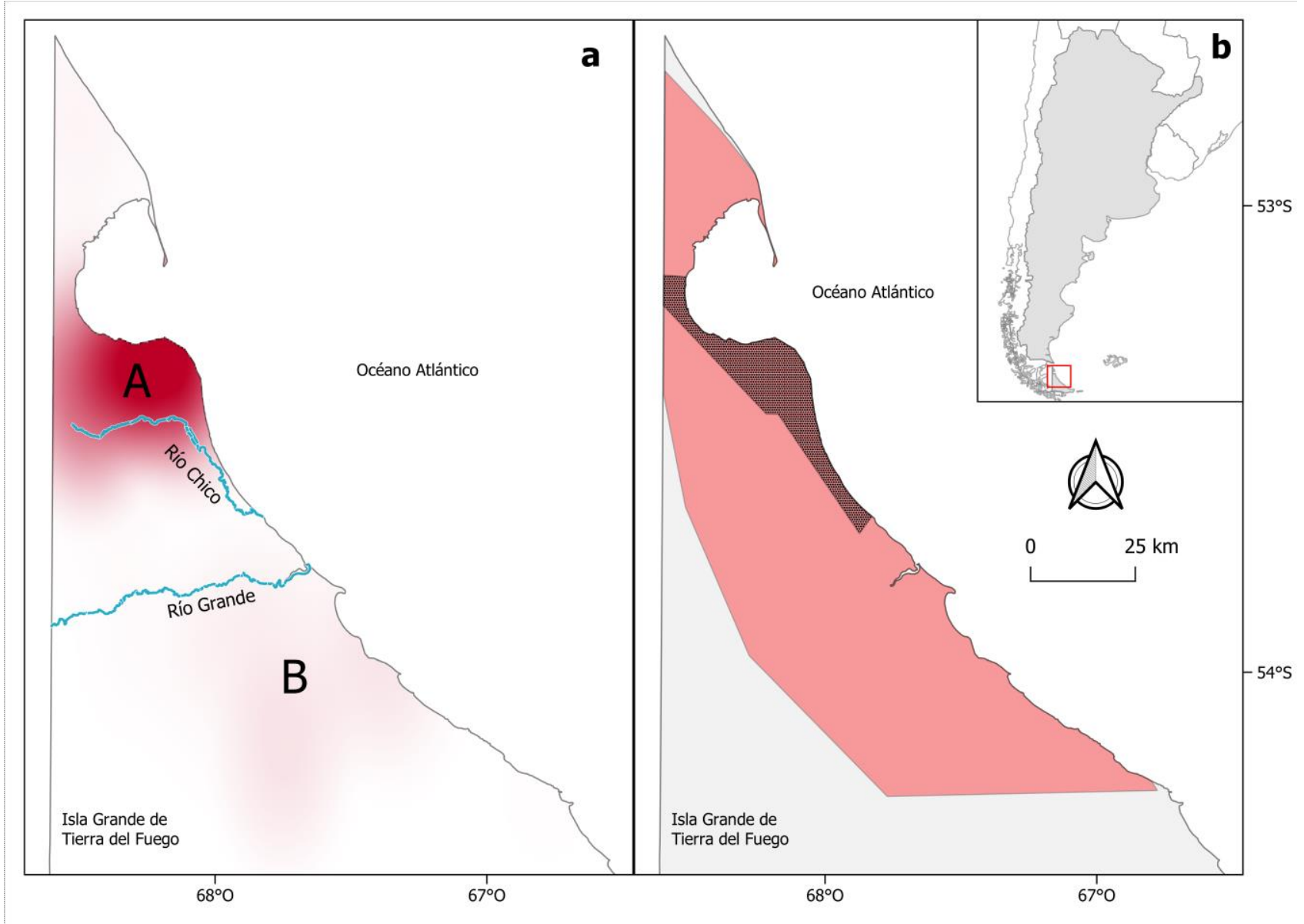

Figura 2.5. a) Mapa de calor generado a partir de frecuencias de evidencias de $C$. villosus en el que pueden distinguirse dos áreas de mayor concentración de evidencias-A: al norte del Río Chico y B al sur del Río Grande. b) Distribución actualizada de C. villosus: en gris y bordes negros se marca la distribución presentada por Poljak et al. (2007) y en rojo la distribución actualizada en esta tesis. 


\subsection{DISCUSIÓN Y CONCLUSIONES}

El trabajo realizado comprende un diseño de muestreo sistemático de la distribución de $C$. villosus en la porción Argentina de la Isla Grande de Tierra del Fuego que permitió realizar una importante actualización de los resultados obtenidos por Poljak et al. (2007). La distribución de C. villosus se incrementó respecto a la distribución estimada por Poljak et al. (2007) y ahora es una especie frecuente en gran parte Norte del sector Argentino de la isla Grande de Tierra del Fuego. La distribución actualizada muestra que el peludo está presente en toda la estepa y en gran parte del ecotono fueguino en Argentina. Si bien el estudio tuvo una buena cobertura este-oeste y norte-sur, los muestreos hacia el sur y sur-oeste se vieron imposibilitados debido a la falta de caminos y de autorizaciones para ingresar a los establecimientos rurales. Igualmente, hacia el sur del ecotono se encuentran suelos inundables y rocosos, condiciones adversas para un mamífero semi-fosorial como el peludo.

Además de la distribución actualizada, resulta interesante identificar aquellos factores del ambiente que tuvieron un papel importante en el establecimiento y posterior dispersión del peludo a lo largo de la Isla Grande. Por lo tanto, conocer si la presencia del peludo en sectores está asociada al tipo de vegetación, tipo de suelo, elevación del terreno y/o a alguna muestra asociación son alguna estructura en el paisaje previamente mencionada como posible facilitadora de la invasión, resulta clave para entender el avance de este armadillo en la isla y así también establecer áreas para el manejo de la especie.

Nuestros resultados son, en gran medida, consistentes con estudios anteriores realizados sobre esta especie de armadillo en su distribución nativa. Por ejemplo, Abba et al. (2007) reportaron que es una especie común en pastizales naturales con suelos húmicos. En Tierra del Fuego, nuestros resultados mostraron que la mayor densidad de signos se encuentra asociada al mismo tipo de suelo húmico. Los suelos húmicos seguramente sean preferidos por su alto contenido de materia orgánica e invertebrados hipogeos (Rodríguez et al., 2004), un ítem importante en la dieta de los armadillos

(Redford, 1985; Abba y Cassini, 2010; Arriagada et al., 2017; Gallo et al., 2019a). Aunque 
las características topográficas de la Patagonia no son directamente comparables con las de la región pampeana, en ambas regiones $C$. villosus parece evitar terrenos inundables (Abba et al., 2012). Así mismo, nuestros resultados muestran que es más probable encontrar peludos en ambientes de vegetación con altura media y alta, (que corresponden a coironales o arbustos de Mata negra o verde, aunque esto no se vio reflejado en el análisis de asociación entre la presencia del peludo y el tipo de vegetación) en los paisajes del norte fueguino. Esto contrasta con los resultados obtenidos por Abba et al. (2016), quienes sugieren que C. villosus prefiere zonas de vegetación más baja. Teniendo en cuenta la baja tasa metabólica de los armadillos y las caracterísicas climáticas del ambiente fueguino, con bajas temperaturas durante todo el año y vientos fríos constantes, es muy probable que el peludo busque refugio en la vegetación de mayor altura. Este comportamiento fue reportado también en otros armadillos y en osos hormigueros, los cuales regulan su actividad en relación a la temperatura del ambiente, buscando sitios de vegetación elevada durante momentos de bajas temperaturas para evitar que la temperatura corporal disminuya rápidamente (ver Camilo-Alves and Mourão, 2006; Layne and Glover, 1985).

En estudios anteriores Poljak et al. (2007) registraron una estrecha relación entre C. villosus y la red de gasoductos de la Isla Grande, los cuales son calentados hasta $4^{\circ} \mathrm{C}$ para poder transportar el gas con mayor facilidad. Dado los hábitos semifosoriales y la baja tasa metabólica de los armadillos, la cual les impide tener un buen control de la temperatura corporal, (McNab, 1980; Boily, 2002; Superina and Boily, 2007), probablemente dicha característica del paisaje fue importante durante las primeras etapas de la invasión del peludo, sin embargo, nuestros resultados no muestran asociación actual entre la presencia de peludos y los gasoductos. Esto significa que las condiciones climáticas extremas del ambiente fueguino no serían, en esta fase, una limitante para la supervivencia y dispersión de $C$. villosus en la Isla Grande de Tierra del Fuego. Estudios previos argumentan que la distribución de algunas especies de armadillos se encuentra limitadas por las condiciones climáticas (McNab, 1980; Taulman y Robbins, 1996), y que esas distribuciones podrían modificarse en relación a los cambios 
ambientales producidos por el calentamiento global (Taulman y Robbins, 1996). Como resultado de ese fenómeno, la temperatura media anual en Patagonia se ha incrementado los últimos años (Thompson, 2002; Villalba et al., 2005). Es entonces posible que el incremento de la temperatura media anual en estos sectores de Patagonia beneficie de alguna manera a la invasión de este armadillo. Por otra lado, si bien no se encontró una asociación directa entre $C$. villosus y los asentamientos humanos, es frecuente encontrar peludos alimentándose en basurales (Abba et al., 2018) y de restos de ganado. Por lo que seguramente en la etapa de establecimiento de la población introducida, éste armadillo aprovecho la presencia de basurales y ganado para obtener alimento (Figura 3.7). Éste escenario y la presencia de gasoductos que favorecieron la termorregulación de $C$. villosus, podrían haber facilitado el aumento y posterior dispersión de la población introducida de armadillos en la Isla.

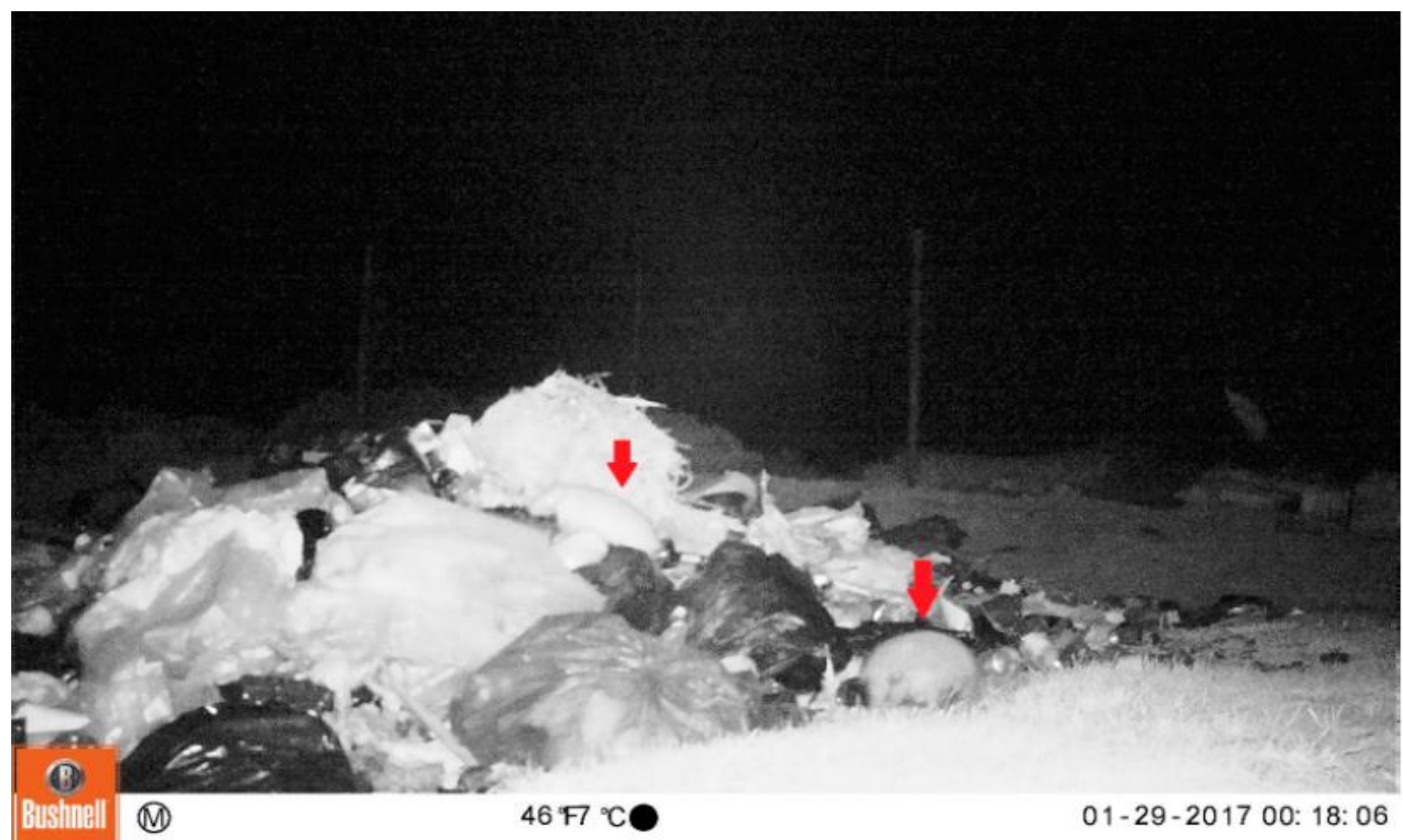

Figura 2.6. Imagen de cámara trampa colocada en el basural de Ea. Cullen, norte de la Isla Grande de Tierra del Fuego. Las flechas muestran dos individuos de Chaetophractus villosus. 
Abba et al. (2014) reportaron que el peludo colonizó Patagonia centro-austral hace aproximadamente 100 años atrás, con una tasa de expansión de 13 km/año (1300 km en 100 años). Los valores de la tasa de expansión calculados en esta tesis para C. villosus en Tierra de Fuego resultaron del mismo orden de magnitud: 10,9 km/año (131 km en 13 años), más allá de la diferencia de escalas empleadas para ambos cálculos y las diferencias en cuanto a los ambientes colonizados en ambos casos. Algo similar ocurre con la velocidad de expansión de Dasypus novemcinctus en América del Norte (promedio histórico de 10,3 km/año; ver (Taulman and Robbins, 1996), lo que podría indicar que la velocidad de expansión media para los armadillos se encontraría dentro de ese rango. Abba et al. (2014) sugirieron que la expansión del peludo a lo largo de Patagonia continental fue favorecida por causas antrópicas como ser el incremento de la producción agricolo-ganadera, y diversas construcciones como caminos, puentes y gasoductos. Además, en el mismo estudio se marca que los ríos son una de las principales barreras naturales para los armadillos Eufractinos, por lo menos para el género Chaetophractus (Abba et al., 2014).

El diseño de muestreo que acompaña los caminos principales, secundarios e internos de los campos permite ver que el avance de los peludos se ha dado en todas direcciones y no parece reconocerse ninguna barrera al movimiento, a excepción del Río Grande. Mientras que al norte del Río Grande la distribución de presencia de peludos parece ser más homogénea, algo diferente se observa al sur del Río Grande. Este río solo tiene dos puentes y ambos se encuentran entre la Ruta 3 y la ciudad de Río Grande. Mientras que el Río Chico, tiene más cruces a lo largo de su curso. Si bien Mella-romero et al. (2020) documentaron el primer registro de nado del peludo, no se trataría de un comportamiento común, siendo el tamaño y la profundidad del cuerpo de agua factores determinantes de dicho comportamiento. Así mismo, es posible que los ríos no sean una barrera infranqueable y que esto dependa de la presencia de puentes, que podrían funcionar como corredores que los armadillos pueden utilizar.

A partir de los resultados obtenidos en estas tesis y los propuestos por otros autores, podemos concluir que los gasoductos favorecieron en una primera etapa a $C$. 
villosus de dos maneras, por un lado debido a la traslocación de armadillos desde el continente por parte de camioneros o transportistas de la industria petrolera, y por otro lado favoreciendo potencialmente la termorregulación al construir sus cuevas cerca de los gasoductos (ver Poljak et al., 2007). Sin embargo, una vez superada esta primera etapa denominada periodo de establecimiento la cual es atravesada por toda especie invasora, la expansión subsecuente a través de la estepa y el ecotono no se vio asociada a los gasoductos.

En cuanto a la abundancia de peludos en la isla, hasta el momento no se han encontrado indicadores que permitan estimar la densidad poblacional a partir de la frecuencia de evidencias indirectas. Por ejemplo, para la mayoría de las especies de armadillos, el número de cuevas no está relacionado con el número de individuos presentes (McDonough et al., 2000). Así mismo, áreas con una alta frecuencia de evidencias indirectas reflejan un alto nivel de actividad. Aunque debería confirmarse la relación entre la frecuencia de signos y la abundancia de individuos, si es así, las áras de mayor concentración de actividad de peludos podrían ser importantes puntos de control o erradicación para esta especie invasora en la Isla Grande.

A partir del análisis de mapas de calor, en esta tesis pudimos identificar dos grandes áreas donde deberían llevarse a cabo planes de manejo. El área A (Bahía San Sebastián, Figura 2.5a) es la de mayor tamaño, allí se encuentra la mayor densidad de cuevas y hozaduras de $C$. villosus. Esto resulta bastante esperable debido a que es la zona donde fueron introducidos los primeros individuos en la década de 1980. Una menor cantidad de signos, pero bastante concentrados, se encontraron en el Área B (alrededores de Río Grande, Figura 2.5a). Si bien se han visto armadillos en esta área, no son tan comunes como en el área A (Cabello et al., 2017, Julio Escobar, pers. com.). La población de individuos en el Área B se estableció más recientemente y podría ser considerada como un frente de invasión. Ambas zonas deberían ser tenidas en cuenta al momento de planificar cualquier plan de control o erradicación de C. villosus. El Área A es un importante sitio de cría para dos especies de cauquenes (Chloephaga spp.) (Canevari, 1996). En este contexto, cualquier mamífero omnívoro invasor puede afectar las 
poblaciones de estas aves que ya se encuentra en grave retroceso. El Área B parece ser la fuente de animales del frente de invasión sur, entonces cualquier esfuerzo de control en esa zona podría ayudar a detener la dispersión de armadillos hacia el ecotono.

La información de la distribución y ecología de $C$. villosus presentada en este Capítulo podría asistir la toma de decisiones enfocadas en el manejo de este armadillo introducido, que suma una nueva especie al ensamble de mamíferos invasores ya presente en la isla. Se sugiere que áreas en los que las zonas con terrenos elevados (naturales o artificiales), con una altura de la vegetación medio/alta (coironales o mata negra/verde) y con suelo del tipo húmico es donde se concentran las mayores niveles de actividad de la especie y que en la Isla Grande de Tierra del Fuego es posible que haya dos áreas bien definidas con mayor concentración de peludos y además barreras (ríos) claves al avance, que podrían ayudar a interrumpir el movimiento de individuos entre áreas encerradas 'entre ríos' y así aislar áreas ya controladas.

\section{BIBLIOGRAFÍA}

ABBA, A.M. and M.H. CASSINI. 2010. Ecological differences between two sympatric species of arma dillos (Xenarthra, Mammalia) in a temperate region of Argentina. Acta Theriologica 55:35-44.

Abba, A.M., M.J. Nabte and D.E. Udrizar SaUthier. 2010. New Data on Armadillos (Xenarthra: Dasypodidae) for Central Patagonia, Argentina. Edentata 11:11-17.

Abba, A.M., S. Poljak, M. Gabrielli, P. Teta and U.F.J. Pardiñas. 2014. Armored invaders in patagonia: recent southward dispersion of armadillos (CINGULATA, DASYPODIDAE. Mastozoologia neotropical 21:311-318.

AbBa, A.M., M.F. Tognelli, V.P. Seitz, J.B. Bender and S.F. VizCaíno. 2012. Distribution of extant xenarthrans (Mammalia: Xenarthra) in Argentina using species distribution models. Mammalia 76:123-136.

Abba, A.M., D.E. Udrizar SaUthier and S.F. Vizcaíno. 2005. Distribution and use of burrows and tunnels of Chaetophractus villosus (Mammalia, Xenarthra) in the Eastern 
Argentinean pampas. Acta theriologica 50:115-124.

AвbA, A. M., AND S. F. Vizcaíno. 2011. Distribución de los armadillos (Xenarthra:

Dasypodidae) en la provincia de Buenos Aires, Argentina. Mastozoología Neotropical 18:185-206.

ABBa, A.M., S.F. VizCAínO and M.H. CASSINI. 2007a. Effects of land use on the distribution of three species of armadillos in the argentinean pampas. Journal of Mammalogy 88:502-507.

ABbA, A.M., S.F. VizCAíno y M.H. CASSINI. 2009. Eto-ecología y conservación de tres especies de armadillos ( Dasypus hybridus, Chaetophractus villosus y C. vellerosus ) en el Noreste de la Provincia de Buenos Aires, Argentina. Edentata 8:41-47.

ABBA, A.M., S.F. VIZCAínO and M.H. CASSINI. 2007b. Effects of land use on the distribution of three species of armadillos in the argentinean pampas. Journal of Mammalogy 88:502-507.

AbBa, A.M., E. Zufiaurre, M. Codesido and D.N. BilenCA. 2015. Burrowing activity by armadillos in agroecosystems of central Argentina: Biogeography, land use, and rainfall effects. Agriculture, Ecosystems and Environment 200:54-61.

Abba, A. M., E. Zufiaurre, M. Codesido, And A. D. N. BilencA. 2016. Habitat use by armadillos in agroecosystems of central Argentina: does plot identity matter? Journal of Mammalogy 20:1-7.

Arriagada, A., L. Baessolo, C. Saucedo, J.e. Crespo, J. Cerda, L. Parra, et al. 2017. Hábitos alimenticios de poblaciones periféricas de Zaedyus pichiy y Chaetophractus villosus (Cingulata, Chlamyphoridae) en la Patagonia chilena. Iheringia. Série Zoologia 107:18.

BolLy P. 2002. Individual variation in metabolic traits of wild nine-banded armadillos (Dasypus novemcinctus), and the aerobic capacity model for the evolution of endothermy. The Journal of experimental biology 205:3207-3214.

Cabello, J.L., A.J. Valenzuela \& C.B. Anderson. 2017. Avance en el proceso de la invasión biológica del peludo Chaetophractus villosus (Dasypodidae) en la Isla Grande de Tierra del Fuego: un nuevo desafío binacional. Anales Instituto Patagonia 45:109_ 
115.

CAMilo-Alves, C. D. S. E. P. Mourão G.D.M. 2006. Responses of a specialized insectivorous mammal (Myrmecophaga tridactyla) to variation in ambient temperature. Biotropica 38:52-56.

CaneVARI, P. 1996. The austral geese (Chloephaga spp.) of Southern Argentina and Chile: a review of its current status. Gibier faune sauvage 13:355-366.

CIUCCIO, M. 2014. Ecología comportamental de los dasipódidos en el pastizal pampeano, con particular consideración de los hábitos alimenticios. Enfoque eco-morfofisiológico. Universidad Nacional del Sur.

Gallo, J.A., L. FAsola and A.M. ABBA. 2019a. Armadillos as pest controllers? Food habits of five armadillos in Argentina. Mastozoologia Neotropical.

Gallo, J.A., S. Poljak, A.M. Abba, D.E. Udrizar Sauthier, E. Camino, R.M. Torres, J. Tamburini, D.M. DeCARre, et al. 2019b. Chaetophractus villosus. P. in: Categorización 2019 de los mamíferos de Argentina según su riesgo de extinción. Lista Roja de los mamíferos de Argentina. (SAyDS-SAREM, ed.).

GRASSÉ, P.P. 1955. Ordre des Edentés, Ordre des Pholidotes. Pp. 1182-1284, in: Traité de Zoologie: anatomie, systématique, biologie. Masson Press, Paris.

Guichón, M.L., M. Monteverde, L. Piudo, J. Sanguinetti y D. Martino. 2016. Mamíferos introducidos en la provincia de Neuquén: Estado actual y prioridades de manejo. LAYNE, J. N., AND D. Glover. 1985. Activity patterns of the common long- nosed armadillo Dasyspus novemcinctus in South-Central Florida. Pp. 407-417 in The evolution and ecology of armadillos, sloth, and vermilinguas (G. G. Montgomery, ed.). Smithsonian Institution Press, Washington, DC.

Mcdonough, C. M., Delaney M.J., P. Quoc L.E., M.S. Blackmore and W.J. Loughry. 2000. Burrow characteristics and habitat associations of armadillos in Brazil and the United States of America. Revista de Biologia Tropical 48:109-120.

MCNAB, B.K. 1980. Energetics and the limits to a temperate distribution in armadillos. Journal of Mammalogy 61:606-627.

Mella-romero, J., J. M. Ávila, F. R. Cortés, And C. M. Villouta. 2020. Registro de nado y 
evidencias indirectas del peludo patagónico Chaetophractus villosus ( Desmarest, 1804 ) en Aysén , Chile. Anales Instituto Patagonia (Chile) 48:47-51.

Moore, D. M. 1983. Flora of Tierra del Fuego. Anthony Nelson Ltd., Shrewsbury, Shropshire, U.K.

Poljak, S., V. Confalonieri, M. Fasanella, M. Gabrielli and M.S. Lizarralde. 2010. Phylogeography of the armadillo Chaetophractus villosus (Dasypodidae Xenarthra): Post-glacial range expansion from Pampas to Patagonia (Argentina). Molecular Phylogenetics and Evolution 55:38-46. Elsevier Inc.

Poljak S., J. Escobar, G. Deferrari and M. LizarRalde. 2007. Un nuevo mamífero introducido en la Tierra del Fuego: El "peludo" Chaetophractus villosus (Mammalia, Dasypodidae) en Isla Grande. Revista Chilena de Historia Natural 80:285-294.

QGIS DeVELOPMENT TEAM. 2017. QGIS Geographic Information System. Open Source Geospatial Foundation Project. <http://qgis.osgeo.org>.

R CORE TEAm. 2017. R: A language and environment for statistical computing. R Foundation for Statistical Computing, Vienna, Austria. <https://www.r-project.org/>.

REDFORD, K.H. 1985. Food habits of armadillos (XENARTHRA: DASYPODIDAE). Pp. 429-437, in: The evolution and ecology of armadillos, sloths and vermilinguas (G. Montgomery, ed.). Smithsonian Institution Press, Washington \& London.

Rimoldi, P.G. y A.M. AвBA. 2013. Nuevos datos de presencia de Chaetophractus villosus en la cuenca del Río Carcarañá, Sur de la Provincia de Santa Fe, Argentina. Edentata $14: 1-8$.

Rodríguez, M., A. France y M. Gerding. 2004. Evaluación de dos cepas del hongo Metarhizium anisopliae var. Anisopliae (metsh.) para el control de larvas de gusano blanco Hylamorpha legans Burm. (COLEOPTERA: SCARABAEIDAE). Agricultura Técnica 64:17-24.

SUPERINA, M. and P. BoILY. 2007. Hibernation and daily torpor in an armadillo, the pichi (Zaedyus pichiy). Comparative Biochemistry and Physiology - A Molecular and Integrative Physiology 148:893-898. 
TAULMAN, J.F. and L.W. ROBBINS. 1996. Recent range expansion and distributional limits of the nine-banded armadillo (Dasypus novemcinctus) in the United States. Journal of Biogeography 23:635-648.

Teta, P., A.M. AbBa, G. CAssini, D. Flores, C. Galliari, S. Lucero, Ramirez, M.A. 2018. Lista revisada de los mamíferos de Argentina. Mastozoología Neotropical 25(1): 163-198.

THOMPSON, D.W.J. 2002. Interpretation of Recent Southern Hemisphere Climate Change. Science 296:895-899.

Villalba, R., M. MasiokAs, T. KitZberger and J. BoninsegnA. 2005. Biogeographical consequences of recent climate changes in the southern Andes of Argentina. Pp. 157-168, in: Global changes and mountain regions (U Huber \& M Reasoner, eds.). Mountain Research Initiative, Switzerland.

WITH, K.A. 2002. The landscape ecology of invasive species. Conservation Biology 16:11921203. 

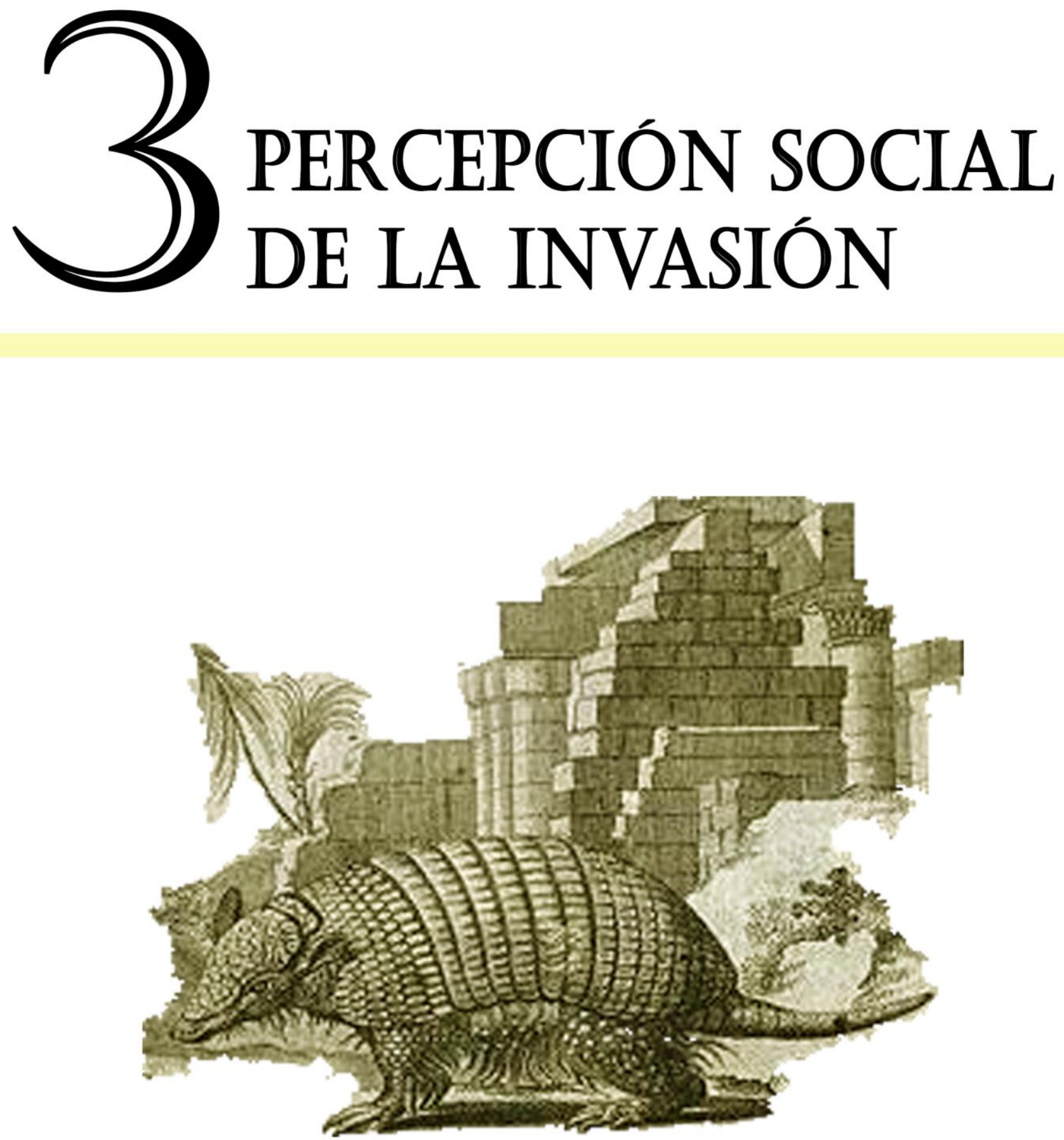

"Tatous" - Natural history, general and particular. Buffon, Georges \& Smellie, William. 1785.

"Caramba mi estomaguito Que lo tengo algo abultao Por comer cuatro quirquinchos $Y$ dos zapallos asaos" 


\subsection{INTRODUCCIÓN}

Las invasiones biológicas han estado asociadas de forma muy estrecha a las actividades humanas, ya sean actuales o históricas. El accionar del ser humano es uno de los factores que operan a distintos momentos del proceso de invasión de una especie, ya sea funcionando como vector (permitiendo o facilitado la dispersión) o tomando las decisiones necesarias para su manejo (García Llorente et al., 2008). De esta manera, una invasión biológica se convierte en una problemática ecológica y socioeconómica (Perrings et al., 2002) y son casos que no pueden ser caracterizados y tratados únicamente desde un punto de vista ecológico sino que debe considerarse también su dimensión humana y social (Kapitza et al., 2019).

Una de las grandes críticas a muchos estudios de invasiones biológicas es la falta de información sobre la percepción social acerca de las especies invasoras (Gobster, 2011). El estudio de la dimensión humana y social de una invasión considera numerosos aspectos que van desde el conocimiento de la especie invasora por parte de la población y el impacto económico que genera hasta las creencias y valores culturales de la población (Figura 3.1; Shackleton et al., 2019).

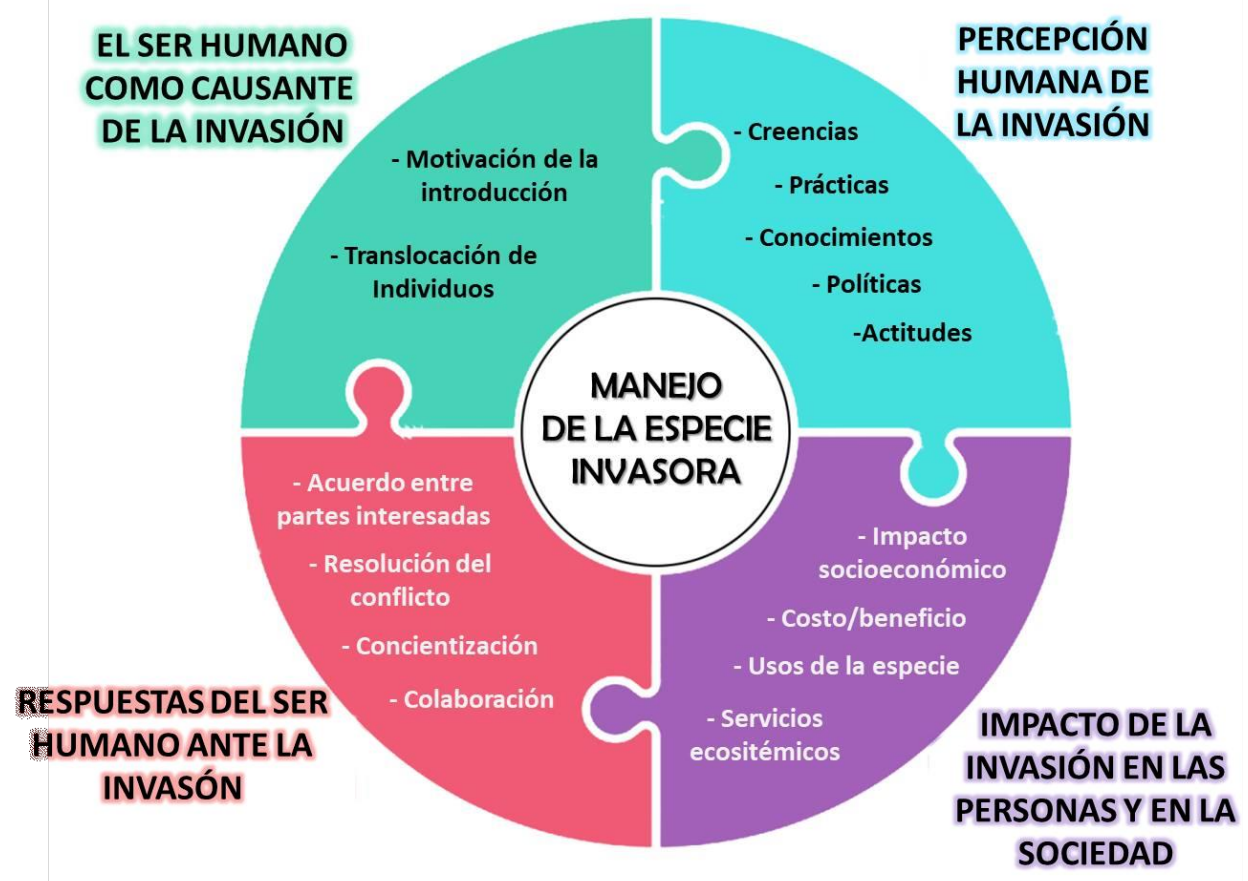

Figura 3.1 Dimensiones humana y social que deben ser consideradas para un buen manejo de una especie invasora. Modificado de Shackleton et al. (2019). 
Una vez que una especie introducida logra establecerse y dispersar en el ambiente invadido, genera cambios en el ecosistema que son impactos que pueden ser tanto a nivel ecológico (ej. pérdida de biodiversidad, modificación de ciclos de nutrientes, etc.) como a nivel económico afectando diversas actividades productivas como la agricultura, la ganadería y la industria forestal (Lovell et al., 2006). Al plantearlo de esta manera, la valoración de cualquier invasión biológica es negativa. Sin embargo, en los últimos años, se ha comenzado a evaluar a las especies invasoras desde un punto de vista cultural. Desde esta mirada social, el impacto de una invasión biológica no siempre es negativo, sino que también puede ser positivo si la especie invasora representa un recurso económico para la sociedad local o permite reformular prácticas culturales (Tapias and Idrovo, 2013). La dimensión social y humana contribuye a una mejor comprensión de la invasión y, por lo tanto, a efectivas prácticas de manejo (Shackleton et al., 2019).

La mayoría de las especies de armadillos representan un componente importante en varias prácticas culturales de las poblaciones rurales y pueblos originarios en su distribución nativa. La caza de armadillos para utilizar su carne como alimento es una práctica muy común (Abba et al., 2010a) y tanto su coraza como su cola son utilizados para la creación de instrumentos musicales, souvenirs o amuletos (Figura 3.2; Peredo, 1999). Por otro lado, en la región pampeana, el peludo suele ser considerado como plaga de los sistemas agropecuarios (Abba et al. 2018). Por un lado, debido a que las cuevas representan un riesgo de lesiones para el ganado (Abba et al., 2005; 2015), y por otro lado, y en mayor medida, porque adecta a la producción de granos almacenados en silos bolsa (Zufiaurre et al. 2019). 

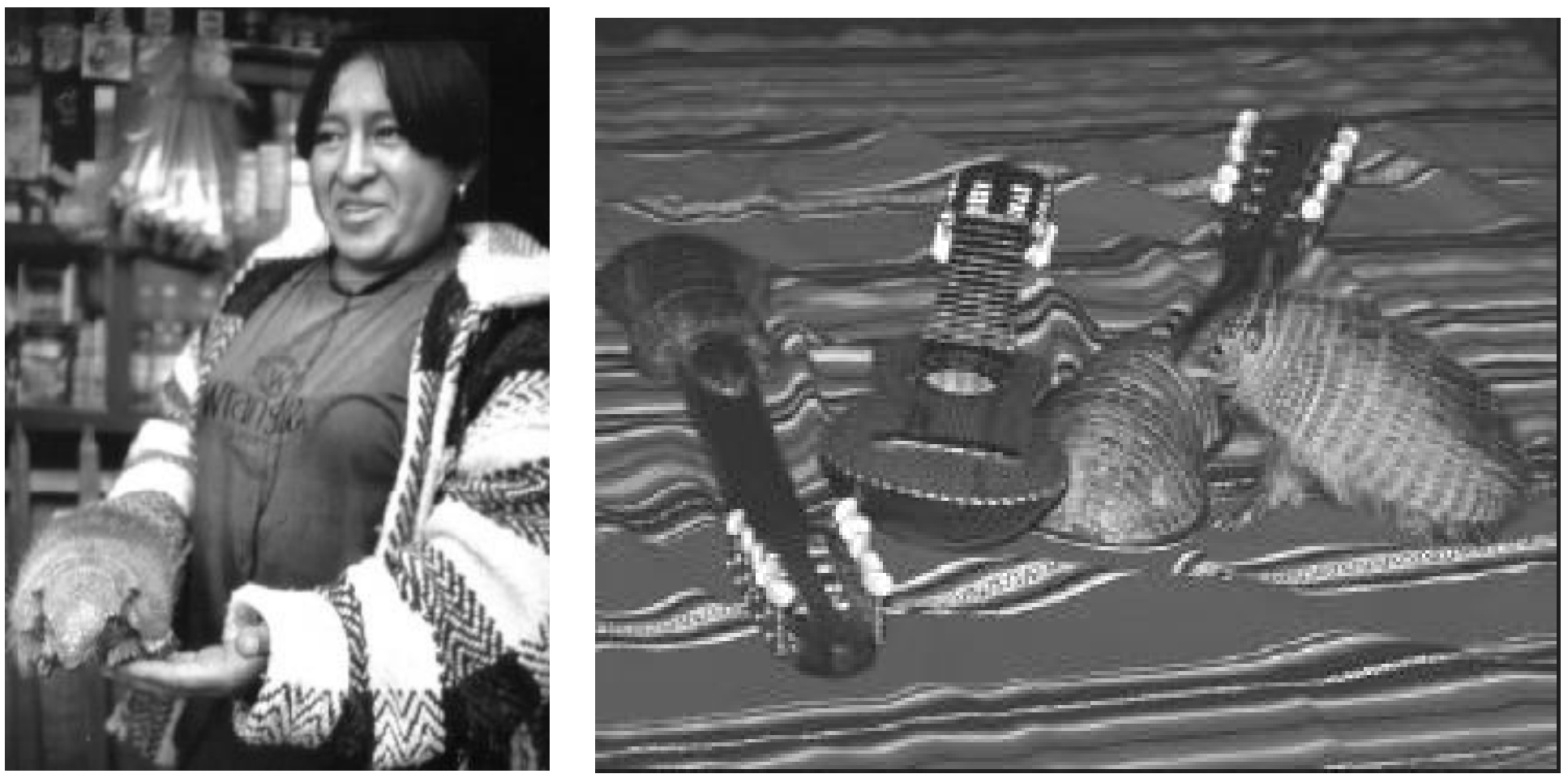

Figura 3.2. Los armadillos disecados se colocan en el hogar como símbolo de buen augurio (izquierda). Instrumentos musicales fabricados con caparazones de armadillos (derecha). Fotografías: Bernardo Peredo/Bolivian photo Agency.

El objetivo de este capítulo fue trabajar en base a un enfoque social para comprender aspectos del proceso de invasión y analizar la percepción por parte de la población fueguina sobre la presencia del peludo en el norte de Tierra del Fuego de manera. De esta manera se obtuvo información acerca de las causas de introducción, tiempos de expansión, impactos y usos de este armadillo en la Isla Grande de Tierra del Fuego.

\subsection{MATERIALES Y MÉTODOS}

Durante febrero de 2018 se llevaron a cabo encuestas a pobladores o trabajadores locales del departamento de Río Grande en la Isla Grande de Tierra del Fuego. Las encuestas fueron realizadas en diferentes estancias de zona de estepa y ecotono (Figura 3.3) a personas que no fueron preseleccionadas, ni elegidas por cargo/rol, sino que se 
entrevistaron a las personas que se encontraron en las propiedades al momento de la visita.

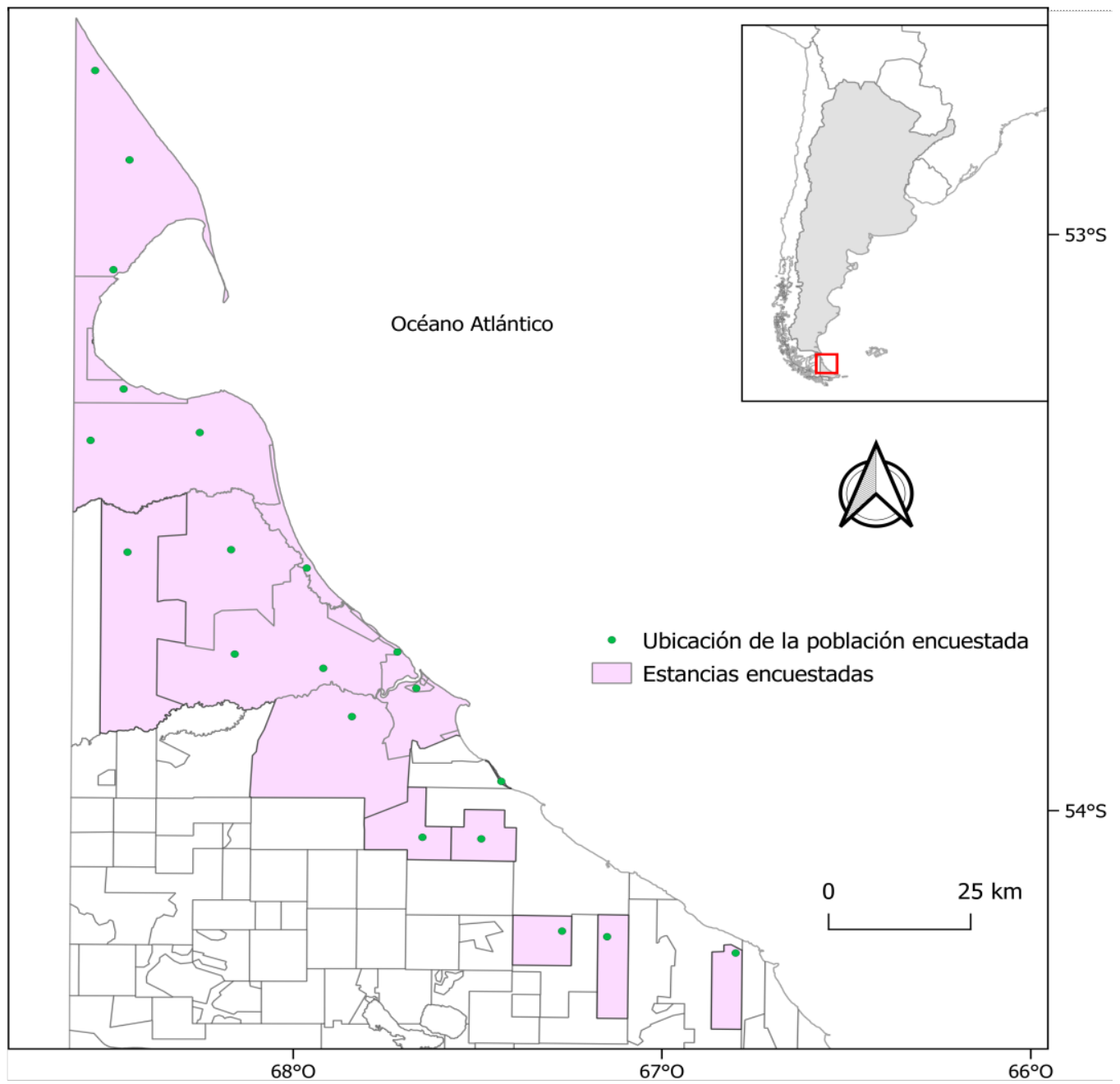

Figura 3.3. Estancias donde se llevaron a cabo las encuestas a pobladores locales.

El departamento posee una población rural de 941 personas que habitan en 106 propiedades rurales (INDEC, censo poblacional nacional, Argentina 2010). La principal actividad de estas propiedades es la cría de ganado ovino y explotación petrolera. En cada entrevista se registró el tiempo desde que la especie fue observada en la zona, posibles causas de introducción, la utilidad que tiene esta especie de armadillo y el posible impacto en las actividades productivas de la región. 
Algunas de las preguntas fueron abiertas y otras fueron cerradas, donde el encuestado debía responder de manera positiva o negativa o elegir opciones de una serie de posibles respuestas, pudiendo agregar nuevas (Figura 3.4). Para el análisis se calcularon proporciones para cada respuesta dada. La información acerca del tiempo de presencia y movilización de peludos en Tierra del Fuego fue incluida en un GIS con el que se construyó un mapa para visualizar espacial y temporalmente la ubicación relativa de las respuestas obtenidas.

\begin{tabular}{|l|l|}
\hline DÍA & Punto GPS \\
\hline \multicolumn{2}{|c|}{ Identificación del Establecimiento } \\
\hline Nombre: & Localidad: \\
\hline Calle/Ruta: & No/ Km: \\
\hline Actividad: & \\
\hline
\end{tabular}

\begin{tabular}{|c|c|c|}
\hline ¿Sabe que hay peludos en la Isla? & $\mathrm{SI}$ & $\mathrm{NO}$ \\
\hline \multicolumn{3}{|l|}{ ¿Suele verlos por la zona? } \\
\hline \multicolumn{3}{|l|}{$\begin{array}{l}\text { ¿Hace cuánto tiempo empezó a verlos } \\
\text { por acá? }\end{array}$} \\
\hline \multicolumn{3}{|l|}{$\begin{array}{l}\text { ¿En qué lugares de la Isla fueron } \\
\text { introducidos por primera vez? }\end{array}$} \\
\hline \multicolumn{3}{|l|}{$\begin{array}{l}\text { ¿Por qué motivo fueron introducidos } \\
\text { los peludos? }\end{array}$} \\
\hline $\begin{array}{l}\text { ¿Sabe si la gente suele movilizar } \\
\text { peludos cuando los ve? }\end{array}$ & $\mathrm{SI}$ & NO \\
\hline ¿Tienen algún uso los peludos? & $\mathrm{SI}$ & NO \\
\hline \multicolumn{3}{|l|}{ ¿Cuál? } \\
\hline \multirow{4}{*}{ ¿Usted los usa para algo? } & \multicolumn{2}{|l|}{ Comida } \\
\hline & \multicolumn{2}{|l|}{ Venta } \\
\hline & \multicolumn{2}{|l|}{ Mascota } \\
\hline & \multicolumn{2}{|l|}{ Otros: } \\
\hline ¿Causan algún problema los peludos? & $\mathrm{SI}$ & NO \\
\hline ¿Cuál? & & \\
\hline \multicolumn{3}{|l|}{ Observaciones: } \\
\hline
\end{tabular}

Figura 3.4. Formato de la entrevista realizada 


\subsection{RESULTADOS}

La población muestreada incluyó 16 personas asociadas a actividades ganaderas (encargados de estancia, puesteros), 2 pescadores y 2 camioneros, todas estas personas vivían, frecuentaban o trabajaban en el lugar hacía más de 30 años. Los 16 ganaderos entrevistados pertenecían todos a distintas propiedades rurales con lo que el relevamiento cubrió el $15 \%$ de las propiedades de toda la isla. Si consideramos la distribución actualizada de C. villosus (Capítulo 2), la cual abarcaría 49 estancias ganaderas entre la estepa y el ecotono, el número de estancias donde se realizaron las entrevistas representa un $46 \%$ de las propiedades dentro del área invadida por el peludo. Los pobladores encuestados fueron, en general, trabajadores que recorren kilómetros de campo a diario, por lo que el peludo es una especie conocida para muchos.

Los resultados más destacados de las entrevistas se pueden reunir en los siguientes puntos:

- El $100 \%$ de los entrevistados conocían a la especie y sabían de la presencia del peludo en la Isla.

- En estancias cercanas a los puntos de introducción (Zona de San Sebastián) se observan peludos hace entre 10 y 30 años (Figura 3.5).

- De las 20 personas entrevistadas, dos aseguran haber movilizado ejemplares al sur del Río Grande en los últimos 5 años (Figura 3.5). 


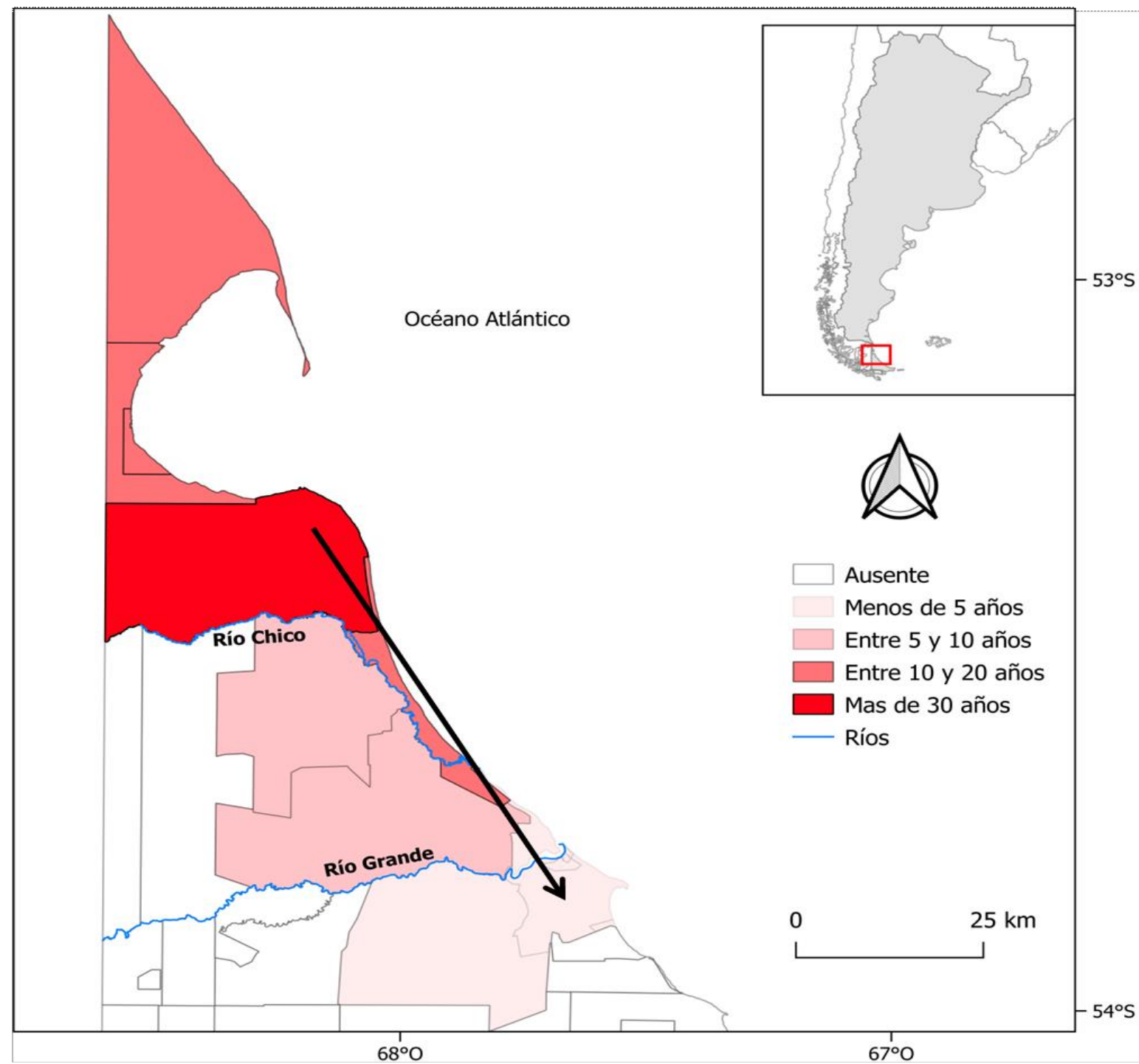

Figura 3.5. Tiempo estimado de presencia del peludo según las respuestas de los entrevistados en cada establecimiento entrevistado. La flecha negra indica la dirección de traslocación de individuos de $C$. villosus.

- Nueve de 20 entrevistados declararon que los "armadillos fueron traídos por camioneros" (Figura 3.6). Otras de las causas de introducción mencionadas por los entrevistados fue la "accidental", 6 de 20 entrevistados optaron por esta causa. Esta categoría se refiere a la introducción de peludos de manera accidental en las tuberías de gas y petróleo que fueron transportadas desde el continente a la Isla durante la construcción de las instalaciones de las industrias petroleras. 
- Trece personas declararon que el uso principal que tiene el peludo, es para alimento. Así mismo, todos coinciden en que es común la gente los atrape y los mantenga en cautiverio para luego usar su carne como alimento (Figura 3.6).

- Seis de 20 entrevistados han mantenido peludos en cautiverio como mascotas (Figura 3.6).

- 14 de los 20 entrevistados indicaron que las cuevas realizadas por C. villosus representan un riesgo de lesiones para el ganado (Figura 3.6).

- 3 de os 20 entrevistados aseguran que los peludos podrían depredar sobre las crías de ganado.
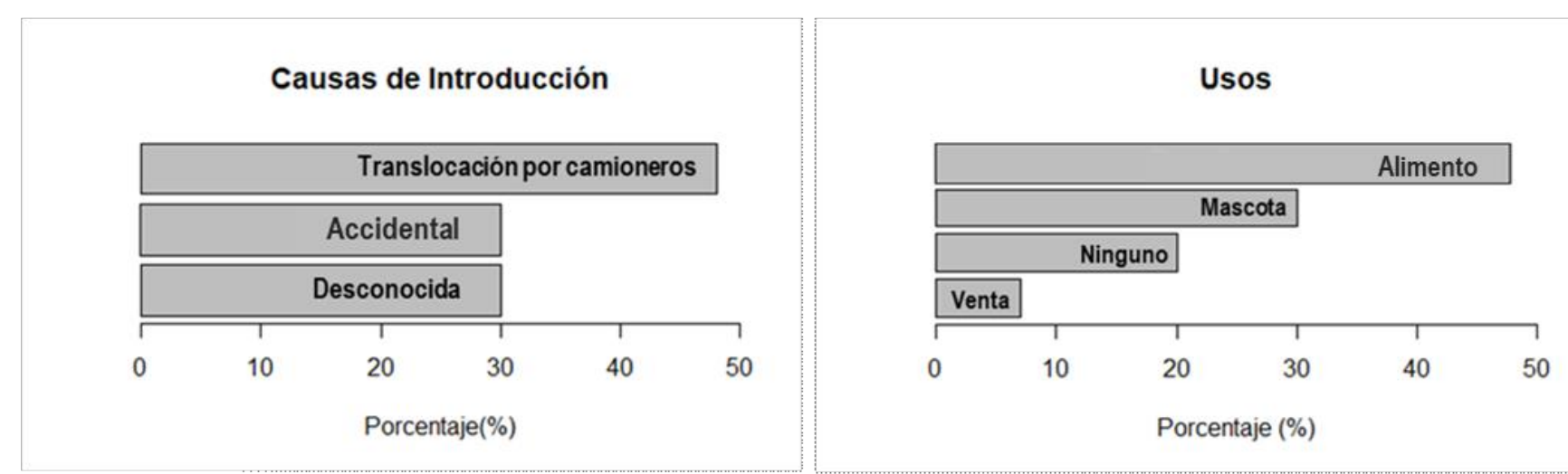

Impacto en Actividades Agropecuarias

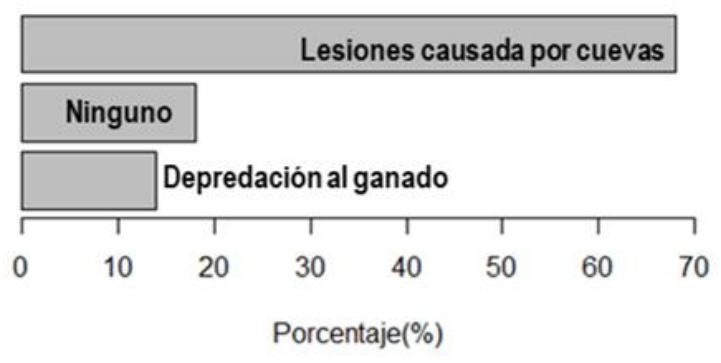

Figura 3.6. Principales resultados de las encuestas realizadas. 


\subsection{CONCLUSIONES}

La dimensión humana y social sobre la invasión del peludo fue analizada desde dos perspectivas. Por un lado se obtuvo información acerca de la invasión de C. villosus (causas de introducción y tiempo de expansión) y por otro, pudimos conocer la percepción de la población local ante la presencia de esta nueva especie en la isla (usos e impactos).

Los ríos son una barrera natural para los armadillos Euphractinae (Abba et al., 2014), y por ende, la dispersión de C. villosus hacia el sur de la Isla Grande se vio limitada por dos ríos (Figura 3.4, Capítulo 2). Sin embargo, a partir de las encuestas realizadas se comprobó que la translocación dentro de la Isla puede ser uno de los mecanismos por los cuales los peludos han podido traspasar esta barrera. Nuestros resultados apoyan que la principal causa de introducción de este armadillo en la Isla fue la translocación de individuos por parte de transportistas de las industrias petroleras desde el continente hacia los puntos de introducción, como ya fue mencionado por Poljak et al., (2007). Lo cual a su vez apoya la hipótesis que solo al comienzo de la invasión los peludos estuvieron asociados a los gasoductos, lo que les permitió una mejor termorregulación (ver Capítulo 2), y no sería casual que los puntos de introducción de C. villosus coincidan con aquellas zonas donde arribaron los transportistas para comenzar a construir las primeras industrias petroleras en la década de 1980 (Poljak et al., 2007). La subsecuente expansión del peludo a lo largo de la estepa y el ecotono fue facilitada por el hombre principalmente debido al transporte de individuos hacia nuevas regiones de la isla, como ser al sur del río grande, donde dos entrevistados aseguraron haber movilizado individuos. Este comportamiento humano es muy común (Guichón et al., 2016) y ha intervenido en numerosas especies invasoras a lo largo de todo el mundo (Lockwood et al., 2005; Hulme, 2009; Guichón et al., 2015), inclusive armadillos (Loughry and McDonough, 2020).

De esta manera, nuestros resultados permiten corroborar la hipótesis que el peludo fue introducido por traslocación intensional desde el continente por parte de transportistas y que su posterior expansión en la Isla Grande no fue solo por dispersión natural sino por nuevas traslocaciones $(n=2)$. 
En su distribución nativa, la relación entre armadillos y humanos data desde tiempos antiguos (Martínez and Gutiérrez, 2004). La principal interacción fue la caza para obtener su carne, hoy en día esta práctica sigue siendo bastante común, inclusive es uno de los motivos del retroceso numérico de varias especies de armadillos (Abba et al., 2010a; Gallo et al., 2019). En la región pampeana, C. villosus produce daños en la producción y hasta es considerado plaga en los sistemas agropecuarios (Abba et al., 2015). Entre otras razones, debido a que la construcción de cuevas representa grandes riesgos para el ganado, ya que pueden producir lesiones en sus extremidades (Abba et al., 2005). En la Isla Grande de Tierra del Fuego, la caza de peludos para alimento o mascotismo parece ser una práctica cultural adquirida por la población rural, siendo uno de los usos más comunes (Figura 3.6). Al igual que en su distribución nativa, el impacto negativo está dado por las cuevas construidas por estos armadillo. Por otra parte la potencial depredación al ganado no parece ser un impacto negativo importante en la Isla Grande sino más bien una creencia cultural debido a que este tipo de comportamientos del peludo ante las crías de ganado recién nacidas o débiles, es frecuente en su distribución nativa. De esta manera, en aquellos establecimientos exclusivamente ganaderos, la presencia de peludos representa un potencial riesgo para el ganado.

La información obtenida en este Capítulo, permitió conocer nuevos aspectos relacionados a la invasión de $C$. villosus. Por un lado, se corroboraron datos obtenidos por Poljak et al. (2007) respecto al tiempo de introducción (mayor a 30 años), a la zona de introducción (San Sebastián) y a la modalidad de introducción. Y por otro lado, se pudo conseguir nueva información con respecto a los usos y al impacto que puede provocar esta especie introducida en las actividades productivas de la región. Además, en relación al tiempo estimado de aparición del peludo en cada establecimiento ganadero, se logró visualizar (Figura 3.5) el movimiento de dispersión de esta especie en relación al tiempo.

Los conocimientos acerca de una invasión biológica que poseen los pobladores locales son relevantes debido a que permiten identificar aquellas especies introducidas que son consideradas "útiles" (porque poseen algún uso en particular) y aquellas que son perjudiciales. Es necesario que los estudios referidos a invasiones biológicas incorporen 
aspectos culturales junto a los biológicos y obtener una visión conjunta de la invasión (Tapias and Idrovo, 2013). Incorporar la dimensión humana y social al estudio de la invasión del peludo en Tierra del Fuego, nos permitió obtener información muy valiosa que podrá ser tenida en cuenta al momento de desarrollar un plan de control o manejo, incorporando la percepción de la población local, sus conocimientos y sus prácticas para poder dirigir cualquier programa de difusión o concientización acerca de esta especie invasora logrando un manejo integral de la invasión de C. villosus.

\section{BIBLIOGRAFÍA}

Abba, A. M., J. A. Gallo, ANd E. Zufiaurre. 2018. Uso de basurales por parte del peludo (Chaetophractus villosus). Notas sobre Mamíferos Sudamericanos 1:1-5.

AbBa, A. M., M. J. Nabte, ANd D. E. U. SAUthier. 2010. New Data on Armadillos (Xenarthra: Dasypodidae) for Central Patagonia, Argentina. Edentata 11:11-17.

Abba, A. M., S. Poljak, M. Gabrielli, P. Teta, and U. F. J. Pardiñas. 2014. Armored invaders in patagonia: recent southward dispersion of armadillos (CINGULATA, DASYPODIDAE. Mastozoologia neotropical 21:311-318.

Abba, A. M., D. E. UdrizAR, ANd S. F. Vizcaíno. 2005. Distribution and use of burrows and tunnels of Chaetophractus villosus ( Mammalia , Xenarthra ) in the Eastern Argentinean pampas. Acta theriologica 50:115-124.

AbBa, A. M., E. Zufiaurre, M. Codesido, and D. N. Bilenca. 2015. Burrowing activity by armadillos in agroecosystems of central Argentina: Biogeography, land use, and rainfall effects. Agriculture, Ecosystems and Environment 200:54-61.

Gallo, J. A. ET AL. 2019. Chaetophractus villosus. Categorización 2019 de los mamíferos de Argentina según su riesgo de extinción. Lista Roja de los mamíferos de Argentina. (SAyDS-SAREM, ed.).

García-Llorente M., B. Martín-López, J.A. González And C. Montes. 2008. Social perceptions of the impacts and benefits of invasive alien species: implications for management. Biological Conservation 141:2969-2983.

GobsteR, P. H. 2011. Factors Affecting People's Responses to Invasive Species Management. Pp. 249-263 in Invasive and Introduced Plants and Animals: Human Perceptions, Attitudes and Approaches to Management Edited (I. D. Rotherham \& R. A. Lambert, eds.). EARTHSCAN, London - Washington, DC. 
Guichón, M. L., V. V. Benitez, A. C. Gozzi, M. HertzRiken, and M. BorgniA. 2015. From a lag in vector activity to a constant increase of translocations: invasion of Callosciurus squirrels in Argentina. Biological Invasions 17:2597-2604.

Guichón, M. L., M. Monteverde, L. Piudo, J. Sanguinetti, And D. Martino. 2016. Mamíferos introducidos en la provincia de Neuquén: Estado actual y prioridades de manejo. Mastozoología Neotropical, 23:255-265.

HuLme, P. E. 2009. Trade, transport and trouble: Managing invasive species pathways in an era of globalization. Journal of Applied Ecology 46:10-18.

INDEC. Censo Nacional de Población, hogares y viviendas. 2010.

Kapitza, K., H. Zimmermann, B. Martín-López, And H. Von Wehrden. 2019. Research on the social perception of invasive species: a systematic literature review. NeoBiota 43:4768.

LOCKWOOD, J. L., P. CASSEY, AND T. BLACKBURN. 2005. The role of propagule pressure in explaining species invasions. Trends in Ecology and Evolution 20:223-228.

Loughry, W. J., And C. M. Mcdonough. 2020. The Nine-Banded Armadillo. University of Oklahoma Press.

LoVelL, S. J., S. F. STONE, AND L. FernANDEZ. 2006. The economic impacts of aquatic invasive species: A review of the literature. Agricultural and Resource Economics Review 35:195-208.

MARtínez, G., AND M. GutiéRREZ. 2004. Tendencias en la explotación humana de la fauna durante el Pleistoceno final y Holoceno en la Región Pampeana (Argentina). Zooarchaeology of South America 1298:81-98.

Peredo, B. 1999. Bolivia's Trade in Hairy Armadillos. TRAFFIC Bulletin 18:41-45.

Perrings, C. ET AL. 2002. Biological invasion risks and the public good: An economic perspective. Ecology and Society 6.

Poljak, S., J. Escobar, G. Deferrari, and M. Lizarralde. 2007. Un nuevo mamífero introducido en la Tierra del Fuego: El "peludo" Chaetophractus villosus (Mammalia, Dasypodidae) en Isla Grande. Revista Chilena de Historia Natural 80:285-294.

Shackleton, R. T., B. M. H. Larson, A. Novoa, D. M. Richardson, and C. A. Kull. 2019. The human and social dimensions of invasion science and management. Journal of Environmental Management 229:1-9. 
TAPIAS M, M., Y V. IDROVO C. 2013. Aportes de la etnobotánica al estudio de las invasiones biológicas. Casos en la región rioplatense (Argentina). Historia Natural 3:61-76.

Zufiaurre E., A. M. AbBA And D. N. BilencA. 2019. Assessment of stakeholder perceptions of the damage to silo bags by vertebrate species in Argentina. Human Dimensions of Wildlife 24:80-86. 


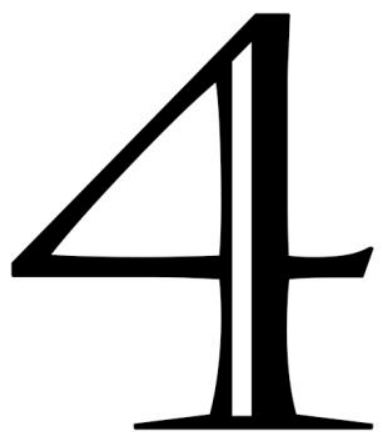

\section{HÁBITOS ALIMENTICIOS E IMPACTO SOBRE ESPECIES AUTÓCTONAS}

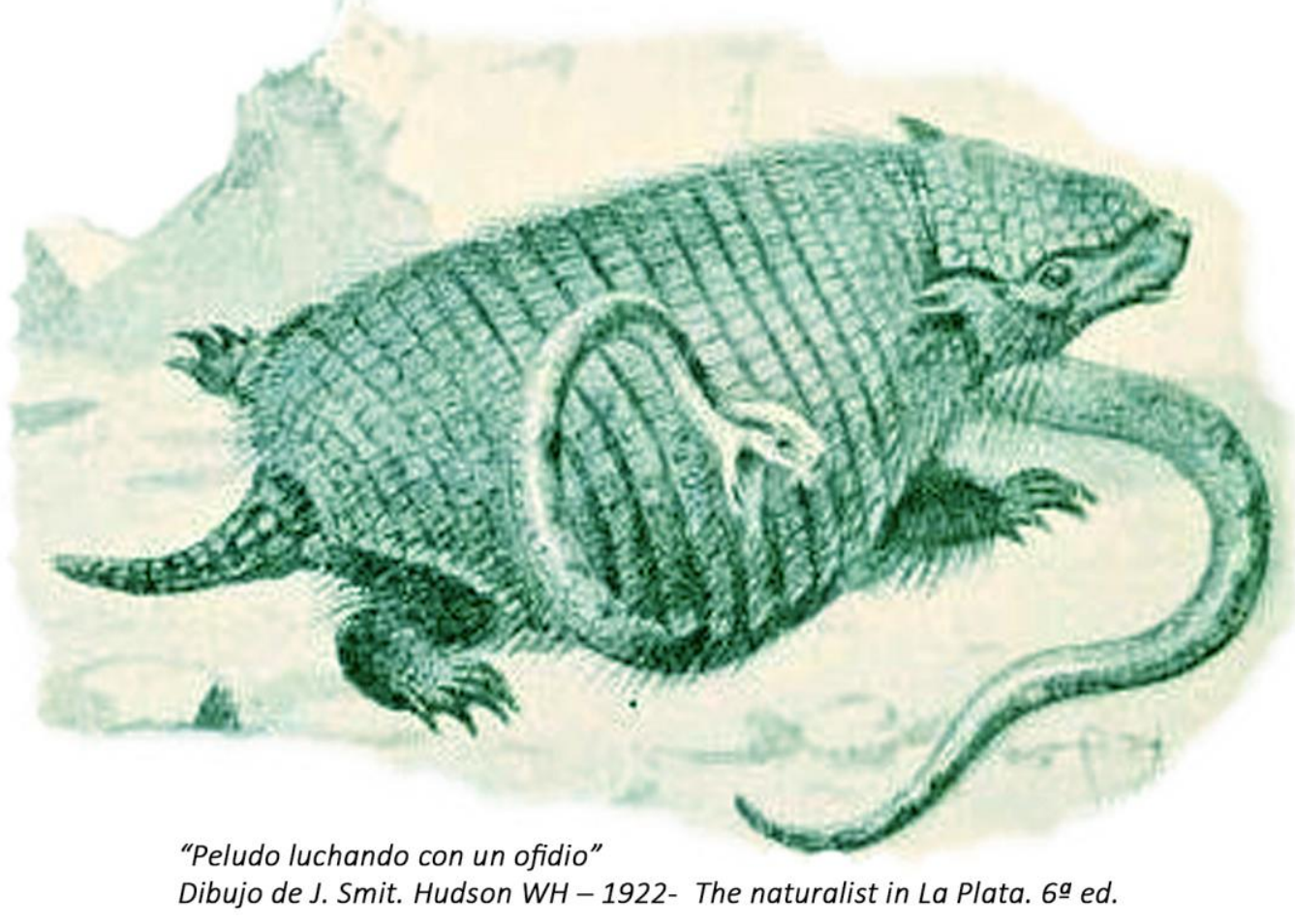

"Agarré un peludo, Marcelina, Agarré un peludo grandote. Estaba comiendo en una usamenta e cordero, ¡Cha, que corría!" 


\subsection{INTRODUCCIÓN}

El conocimiento de los hábitos alimenticios de cualquier especie es fundamental para entender aspectos de su ecología (Johnson, 1980; Gallina-Tessaro, 2011). No solo desde el punto de vista energético, sino también para comprender las interacciones entre los consumidores y su ambiente (depredación, competencia, etc.; Sih and Christensen, 2001). Para el estudio de una invasión biológica, conocer la dieta de las especies introducidas es importante para su manejo ya que permite determinar el uso del ambiente, su rol ecológico, las modificaciones sobre las redes tróficas y su impacto sobre especies nativas (Hernández et al., 2017; Kamenova et al., 2017).

Los hábitos alimenticios de los armadillos se encuentran asociados a sus características morfológicas. Sus cortos miembros con fuertes garras les permiten aplicar la fuerza necesaria para poder perforar el sustrato (Redford, 1985). Las estructuras resultantes de esta actividad corresponden a dos tipos: estructuras simples conocidas como "hozaduras", las cuales son producto de la búsqueda de alimento, y estructuras complejas como las cuevas (Abba et al., 2005). Los armadillos poseen una dieta muy variable, desde insectos y plantas hasta pequeños vertebrados como roedores y serpientes (McDonough and Loughry, 2008). Sin embargo, algunos armadillos de la tribu Euphractinae, dentro de la cual se encuentra Chaetophractus villosus, tienen una dieta mucho más amplia que el resto, seguramente como adaptación a ambientes donde la diversidad de insectos escasea (Redford, 1985).

Chaetophractus villosus habita prácticamente en todos los ambientes del país y es considerado omnívoro-carnívoro (Redford, 1985). Si bien el peludo es conocido por su alimentación carroñera (Figura 4.1; Arriagada et al., 2017), Gallo et al. (2019) detectaron la presencia de vertebrados enteros en su contenido estomacal y restos de cascaras de huevos, lo que sugiere que este armadillo puede depredar activamente sobre medianos y pequeños vertebrados. En zonas de producción agropecuaria, los estancieros y trabajadores rurales aseguran que es muy común encontrar peludos intentando depredar sobre terneros o corderos recién nacidos (ver Capitulo 3). 


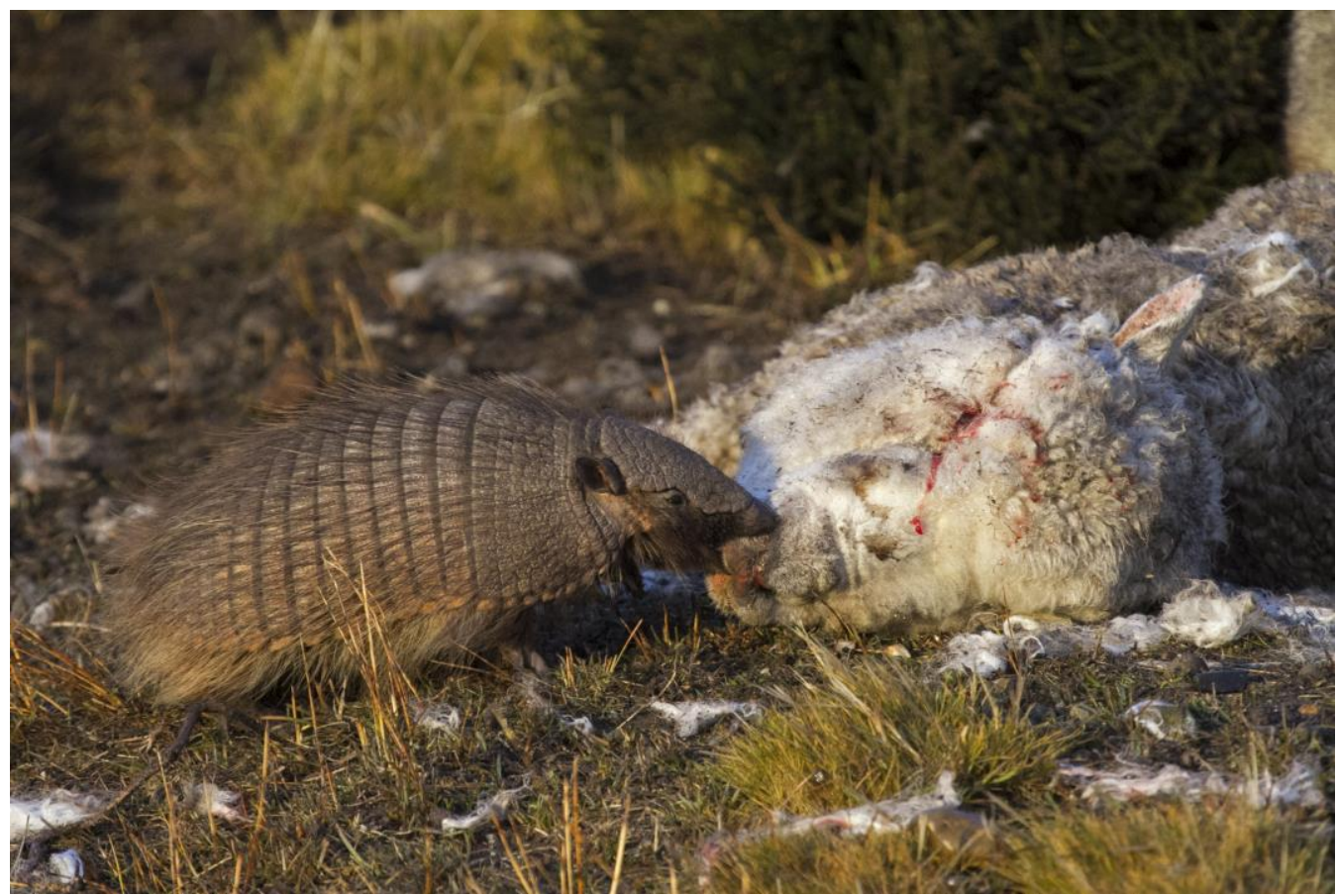

Figura 4.1. Chaetophractus villosus alimentándose de carroña de oveja en la estepa Patagónica de Santa Cruz. Fotografía de Mariano Bertinat.

Los hábitos alimenticios que posee $C$. villosus podrían significar la presencia de un potencial "depredador" de vertebrados en la Isla Grande de Tierra del Fuego. Los depredadores exóticos son una de las tantas causas de retroceso númerico en poblaciones de aves (Groombridge, 1992; Dickman, 1996; Salo et al., 2007; Lyons et al., 2015). La depredación sobre nidos es un factor determinante para el éxito reproductivo de las aves, afectando los ciclos reproductivos y la dinámica de las poblaciones (Arbeiter and Franke, 2018). Aquellas aves que nidifican en el suelo son las más afectadas por depredadores generalistas introducidos, debido al fácil acceso a los nidos (Martin, 1993). La recuperación de las poblaciones de aves migratorias en peligro de extinción se encuentra muy limitada por la baja tasa reproductiva resultante de la depredación de nidos (Klug et al., 2009). Y este problema se intensifica aún más si el ambiente invadido es una isla, como el caso de la Isla Grande de Tierra del Fuego. Muy pocos ecosistemas insulares logran recuperarse luego del impacto generado por depredadores invasores (Salo et al., 2007). 
Los cauquenes (Chloephaga spp.) son un grupo de aves endémico del centro y sur de Sudamérica. Las poblaciones continentales de cauquén común (Chloephaga picta) y cauquén colorado (Chloephaga rubidiceps), y el cauquén real (C. poliocephala) son migratorias. La estepa magallánica en la Isla Grande de Tierra del Fuego es un sitio de reproducción importante para estas especies de cauquenes (Canevari, 1996). Las tres especies se encuentran en peligro de extinción en Argentina (SAyDA y AA, 2017): el cauquén colorado está categorizado como "en peligro crítico" mientras que el cauquén real como "amenazado" y el cauquén común "vulnerable". Una de las principales razones del retroceso numérico en las poblaciones de cauquenes, principalmente de cauquén colorado, son los depredadores introducidos en la Isla como el zorro gris (Lycalopex griseus) y el visón americano (Neovison vison) (Cossa et al., 2017). Con la presencia de Chaetophractus villosus en Tierra del Fuego, se sumaría una nueva especie introducida al ensamble de mamíferos depredadores de la Isla, lo que podría agravar la situación poblacional de los cauquenes. Entender el impacto de los depredadores invasores es importante para asegurar un manejo eficiente del problema (Salo et al., 2007).

Las poblaciones de aves no serían las únicas afectadas por los depredadores introducidos en la Isla Grande de Tierra del Fuego. Aunque poco estudiado en la Isla se encuentran dos endemismos de vertebrados importantes, la lagartija austral, (Liolaemus magellanicus) y el tuco-tuco magallánico (Ctenomys magellanicus) cuyos rangos de distribución coinciden con la del peludo (Ver capítulo 2; Lavilla et al., 2000; Sánchez y Lizarralde, 2019).

Por lo general, en las islas la disponibilidad de recursos durante todo el año es más variable que en el continente ya que están sujetas a cambios estacionales más marcadas (Ruffino et al., 2011). Por estas razones es esperable que la dieta de la población invasora de $C$. villosus presente cambios en relación al continente, en respuesta a la disponibilidad de presas en la Isla Grande de Tierra del Fuego.

El objetivo de este capítulo es estudiar la dieta del peludo en Tierra del Fuego, compararla con la dieta de la población nativa de C. villosus en parte de Patagonia 
continental, y por último evaluar el impacto del peludo sobre aves nativas que nidifican en el suelo y otros vertebrados de la Isla Grande de Tierra del Fuego.

\subsection{MATERIALES Y MÉTODOS}

\subsubsection{Análisis del contenido estomacal}

Durante las temporadas de verano de 2017, 2018 y 2019 se colectaron 34 individuos atropellados de C. villosus en parte de la estepa patagónica continental (Chubut y Santa Cruz; Figura 4.2). En la Isla Grande de Tierra del Fuego se capturaron 12 individuos en la zona de San Sebastián, donde se pudo observar mayor densidad de evidencias indirectas. Cada ejemplar fue sacrificado utilizando una solución eutanásica intracardíaca de Euthanyle ${ }^{\circledR}$, según las normativas legales de colecta científica vigente y con los permisos pertinentes de la Secretaría de Ambiente, Desarrollo Sostenible y Cambio Climático de la Provincia de Tierra del Fuego (Resolución 0064/2017). Los estómagos fueron fijados en una solución de formol al $10 \%$ y conservados en alcohol al $70 \%$. El contenido estomacal fue lavado en un tamiz con malla de $0,1 \mathrm{~mm}$ y colocado bajo lupa binocular Lancet 10x-40x para su identificación. Cada ítem presa fue identificado al nivel de Orden/Familia usando bibliografía específica (Stehr, 1987, 1991). 


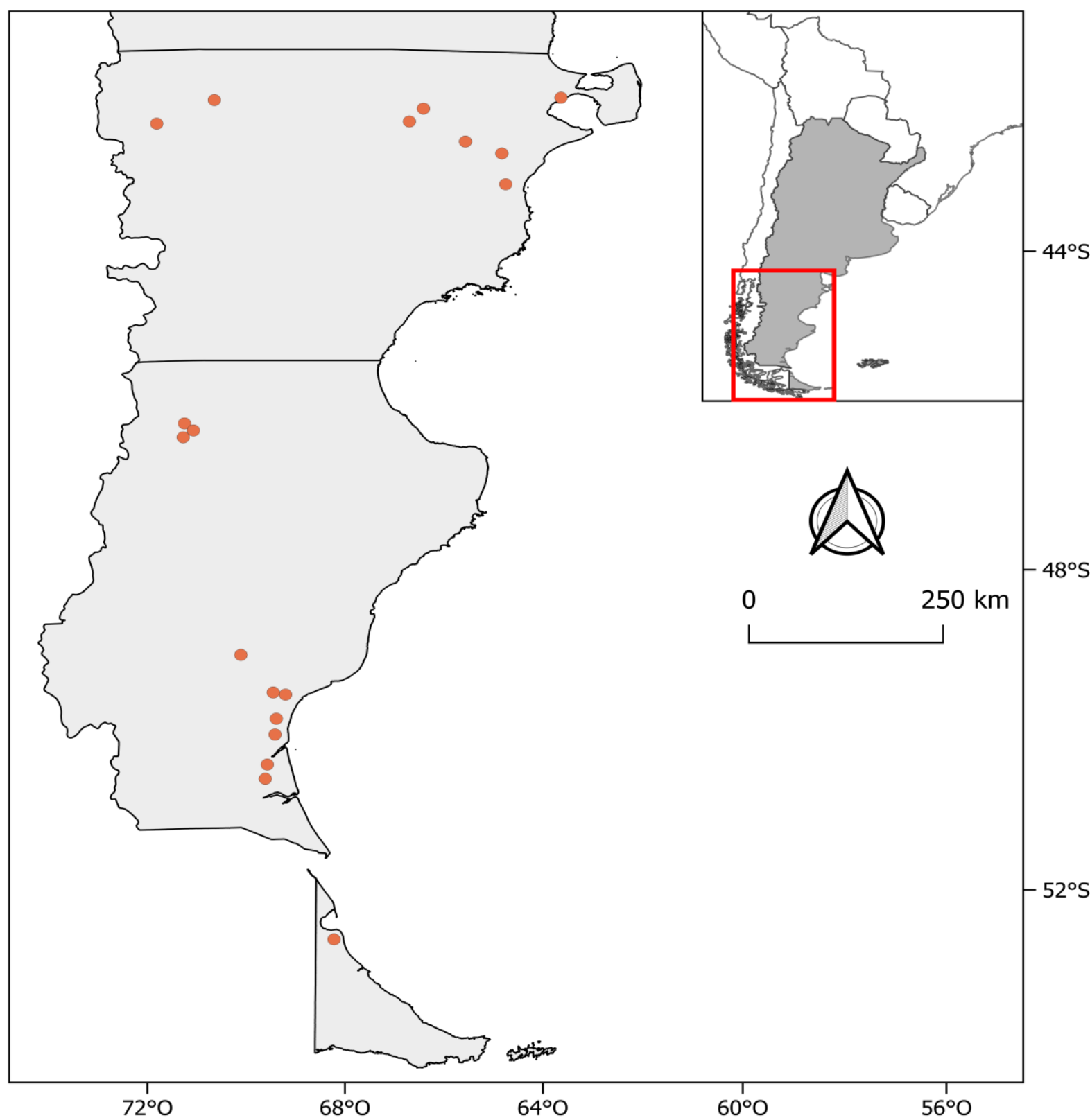

Figura 4.2. Localidades donde fueron recolectados ejemplares de Chaetophractus villosus.

\subsubsection{Análisis estadísticos}

Para el análisis se estableció la siguiente escala de abundancia relativa $(A)$ a la que se asoció un valor de 5 a 0 para su tratamiento estadístico: 5 (Muy abundante,80\% 100\%), 4 (abundante, 60\%- 80\%), 3 (común, 40\% - 60\%), 2 (escaso, 20\% - 40\%), 1 (muy 
escaso $10 \%$ - 20\%) y 0 (ausente, 0\%) (Grosman et al., 1995). Se calculó la frecuencia de ocurrencia de cada ítem (F) como el porcentaje de estómagos en que aparece un determinado ítem alimenticio (Gallina-Tessaro, 2011). Se obtuvo el índice de diversidad de Shannon-Weaver:

\section{$H^{\prime}=-\Sigma[n i / N(3,3219 \log n i / N)]$}

Donde $\mathrm{n}_{\mathrm{i}}$ es el números de individuos de la presa $\mathrm{i}, \mathrm{N}=$ el total de ítems presas consumidas y 3,3219 es el factor de conversión de $\log _{2}$ a $\log _{10}$. Con los tres parámetros mencionados ( $\mathrm{A}, \mathrm{F}$ y H') se calculó el Índice de Categorización de Ítems (ICI):

$$
\mathbf{I C l}=\sqrt{(\mathbf{F x A}) / \mathbf{H}^{\prime}}
$$

Éste índice permite clasificar los ítems presa según el siguiente orden de importancia: Primario ( $\mathrm{ICl}>10)$, Secundario ( $\mathrm{ICl}=5-10)$, Terciario $(\mathrm{ICl}=1-5)$ o Accidental $(\mathrm{ICl}<1)$ (Grosman et al., 1995). El índice muestra la relación entre la frecuencia de ocurrencia, la abundancia relativa y la diversidad de la dieta y categoriza cada ítem dentro de una jerarquía relativa (Grosman, 1995; Mancini and Grosman, 1998; Grosman et al., 2001, 2002).

\subsubsection{Depredación de C. villosus sobre aves nativas}

Durante la época reproductiva de las aves (primavera-verano), en la Isla Grande de Tierra del Fuego, se colocaron nidos artificiales en la misma zona donde se colectaron individuos para el analisis de contenido estomacal para evaluar la depredación por parte de los armadillos sobre aves nativas. Los nidos artificiales se construyeron con material (plumón) de nidos abandonados de cauquén, los cuales se reacomodaron en tamaño (30 $\mathrm{cm}$ de diámetro externo y $6 \mathrm{~cm}$ de profundidad) y se ubicaron cuidando la apariencia con la vegetación circundante. En cada nido se colocaron dos huevos de gallina. Se instalaron un total de 8 nidos artificiales, cada uno con una cámara trampa dispuesta a $5 \mathrm{~m}$ de distancia (Figur 4.4). Las cámaras (modelo Bushnell HD max) se configuraron para tomar fotografías y grabar un vídeo de $10 \mathrm{seg}$. cada vez que se detecaba un movimiento y el intervalo de deteccción se configuró cada $10 \mathrm{seg}$. Los 8 nidos se colocaron al mismo tiempo y las cámaras se mantuvieron activas por un total de 8 días y los huevos eran repuestos cada dos días. 

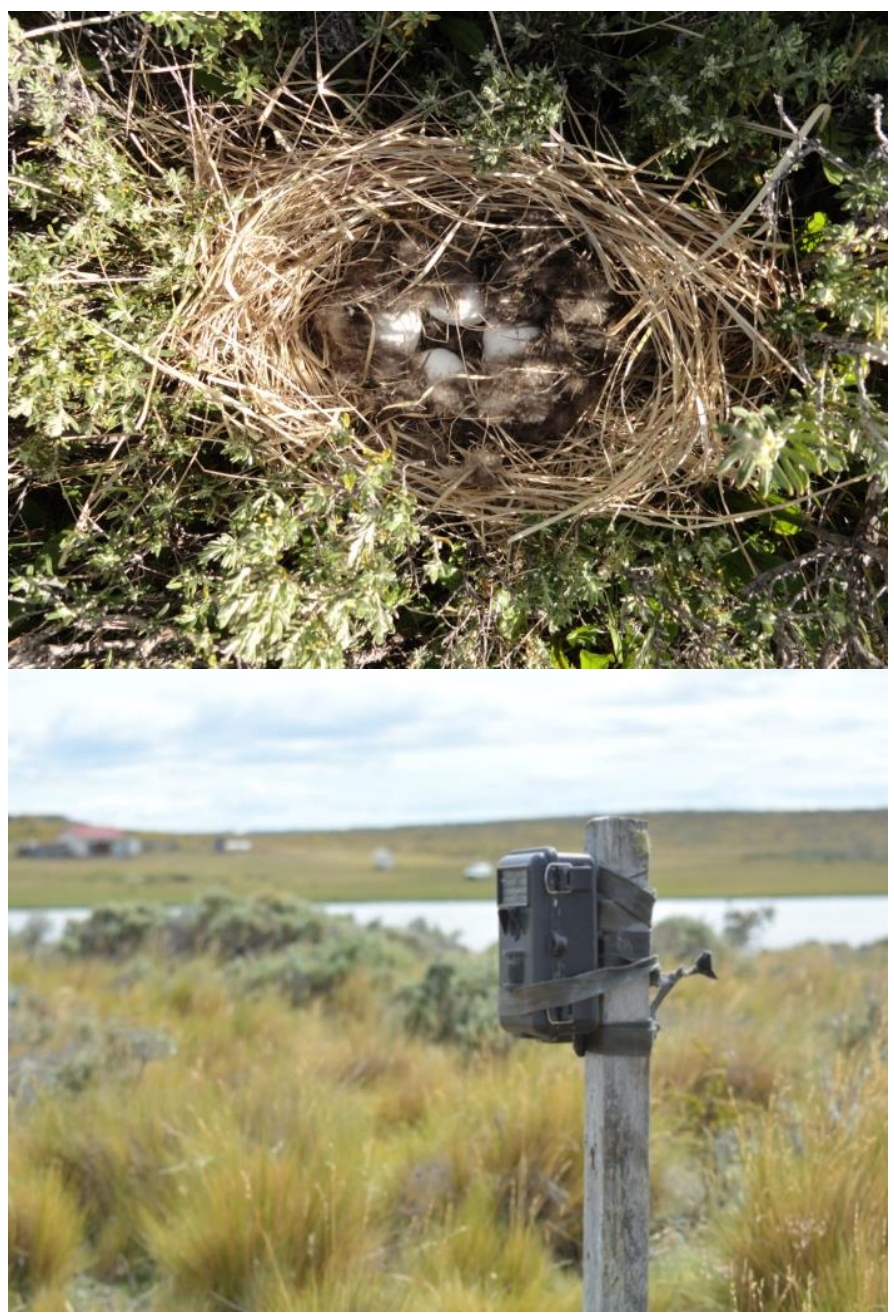

Figura 4.4. Nido artificial (arriba) y cámara trampa (abajo) utilizados para el análisis de depredación de Chaetophractus villosus sobre aves que nidifican en el suelo.

\subsection{RESULTADOS}

\subsubsection{Dieta de Chaetophractus villosus en Patagonia continental}

Se encontraron 10 ítems presa en los ejemplares analizados de $C$. villosus en su distribución Patagónica continental (Figura 4.5, Tabla 4.1), los cuales corresponden a 4 órdenes de insectos, restos de material vegetal (semillas y raíces principalmente), carroña (restos de tejidos de vertebrados) y cáscaras de huevo de una especie de cauquen (Chloephaga sp.). 


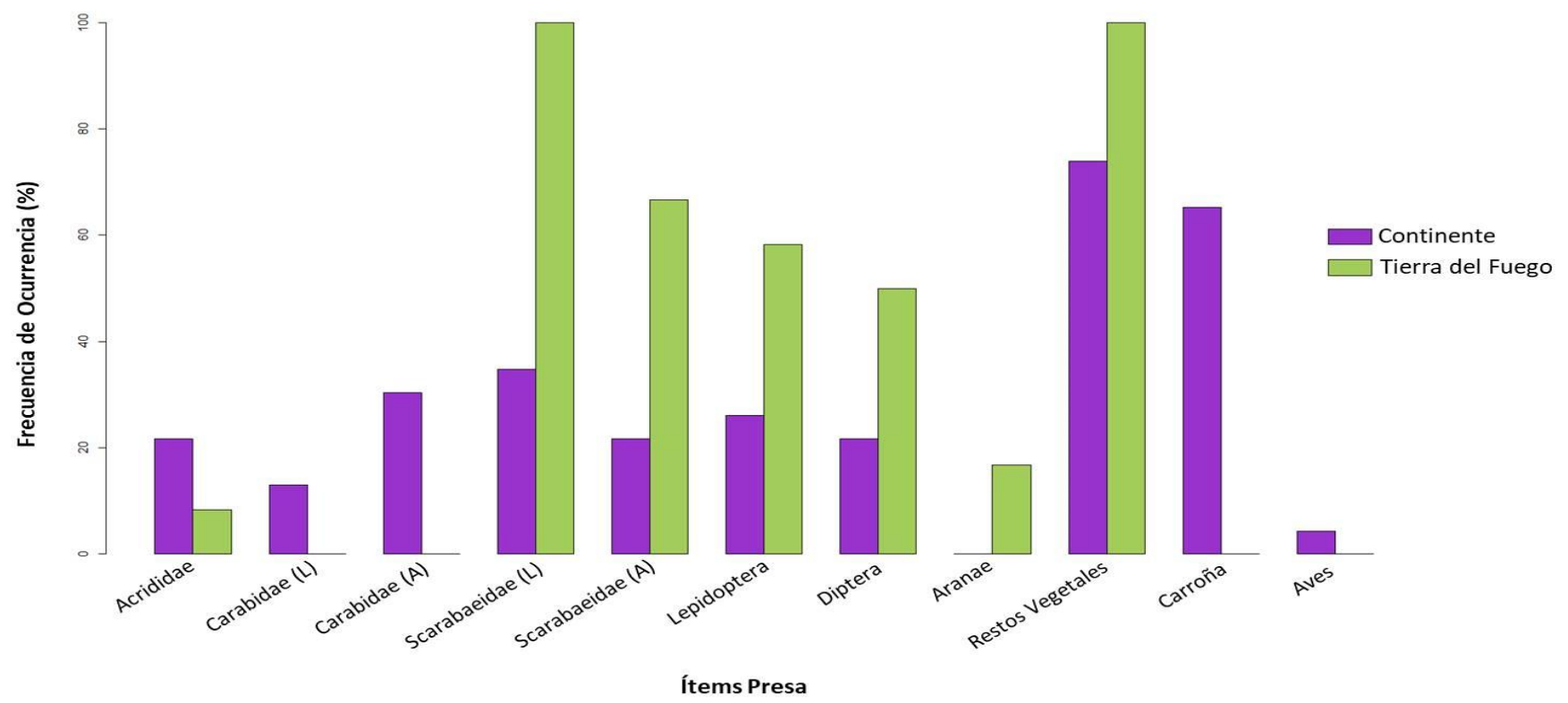

Figura 4.5. Frecuencias de ocurrencia de los ítems alimenticios de Chaetophractus villosus encontrados en las poblaciones del continente (nativa) y de Tierra del Fuego (Invasora). "L" y " $A$ " indican Larva y Adulto, respectivamente.

Los ítems de mayor ocurrencia (>50\%) corresponden a restos vegetales y carroña, mientras que el resto se mantiene por debajo del 50\%. Según el Índice de Categorización de Ítems (ICI), no se encontraron ítems Primarios y solo la carroña fue clasificada como Secundario (Tabla 4.1). Por otro lado, los Restos vegetales y los insectos, excepto las larvas de Carabidae, fueron categorizados como Terciarios. Los huevos de aves se clasificaron como presas ocasionales. 
Tabla 4.1. Valores del Índice de Categorización de Ítems (ICI) de cada presa para las poblaciones nativa (Patagonia continental) e introducida (Tierra del Fuego) de Chaetophractus villosus. Se indican el $\mathrm{N}$ (número de muestras analizadas) y el $\mathrm{H}^{\prime}$ (índice de diversidad) para el área continental y Tierra del fuego. ' $\mathrm{L}$ ' $y$ ' $A$ ' indican larva y adulto, respectivamente.

\begin{tabular}{|c|c|c|}
\hline \multirow{3}{*}{ Orden/Familia } & \multicolumn{2}{|c|}{ Poblaciones de C. villosus } \\
\hline & $\begin{array}{c}\text { Patagonia } \\
\text { continental } \\
(\mathrm{N}=34)\left(\mathrm{H}^{\prime}=2,7\right)\end{array}$ & $\begin{array}{l}\text { Tierra del Fuego } \\
(\mathrm{N}=12) \quad\left(H^{\prime}=1,9\right)\end{array}$ \\
\hline & ICI & ICI \\
\hline ORTHOPTERA & $1,1(\mathrm{~T})$ & $1,05(\mathrm{~T})$ \\
\hline \multicolumn{3}{|l|}{ COLEOPTERA } \\
\hline Carabidae (L) & $0,7(A)$ & - \\
\hline Carabidae (A) & $1,57(\mathrm{~T})$ & - \\
\hline Scarabaeidae (L) & $1,47(\mathrm{~T})$ & $10,93(P)$ \\
\hline $\begin{array}{l}\text { Scarabaeidae } \\
\text { (A) }\end{array}$ & $1,1(T)$ & $3,64(T)$ \\
\hline LEPIDOPTERA & $1,34(\mathrm{~T})$ & $3,59(\mathrm{~T})$ \\
\hline DIPTERA & $1,1(T)$ & $2,58(\mathrm{~T})$ \\
\hline ARANAE & - & $1,05(A)$ \\
\hline $\begin{array}{l}\text { RESTOS } \\
\text { VEGETALES }\end{array}$ & $4,39(\mathrm{~T})$ & $8,42(S)$ \\
\hline CARROÑA & $5,14(S)$ & - \\
\hline AVES & $0,28(A)$ & - \\
\hline
\end{tabular}

\subsubsection{Dieta de Chaetophractus villosus en Tierra del Fuego}

En los 12 estomagos analizados, se encontraron siete ítems presas diferentes (figura 4.5; Tabla 4.1) que corresponden a 3 órdenes de insectos, 1 de arácnidos y a restos vegeteles indeterminados (predominantemente raíces y tubérculos). Se observa que ambas poblaciones comparten 6 tipos de ítems presa, observándose un aumento en la frecuencia de ocurrencia de la mayoria, excepto por el ítem Acrididae. 
$\mathrm{El} \mathrm{ICl}$ indica una marcada tendencia al consumo de items hipógeos (subterrneos) como ser las larvas de Scarabaeideae (ítem Primario) y restos vegetales (ítem Secundario). Los insectos restantes se categorizaron como items Terciarios y las arañas entraron en la categoria de ocasionales.

\subsubsection{Depredación de Chaetophractus villosus sobre aves nativas en Tierra del}

\section{Fuego}

De los 8 nidos artificiales colocados y monitoreados con cámaras remotas ninguno fue depredado por peludos. Solo una de las camaras detectó la presencia de armadillos (Figura 4.6) pero no se detectó depredación. Ese nido y otros 4 fueron depredados por otro mamífero introducido, el zorro gris (Lycalopex gymnocercus) (Figura 4.6, Figura 4.7). En total se registraron 5 eventos de depredación por zorro gris en 5 nidos diferentes. 

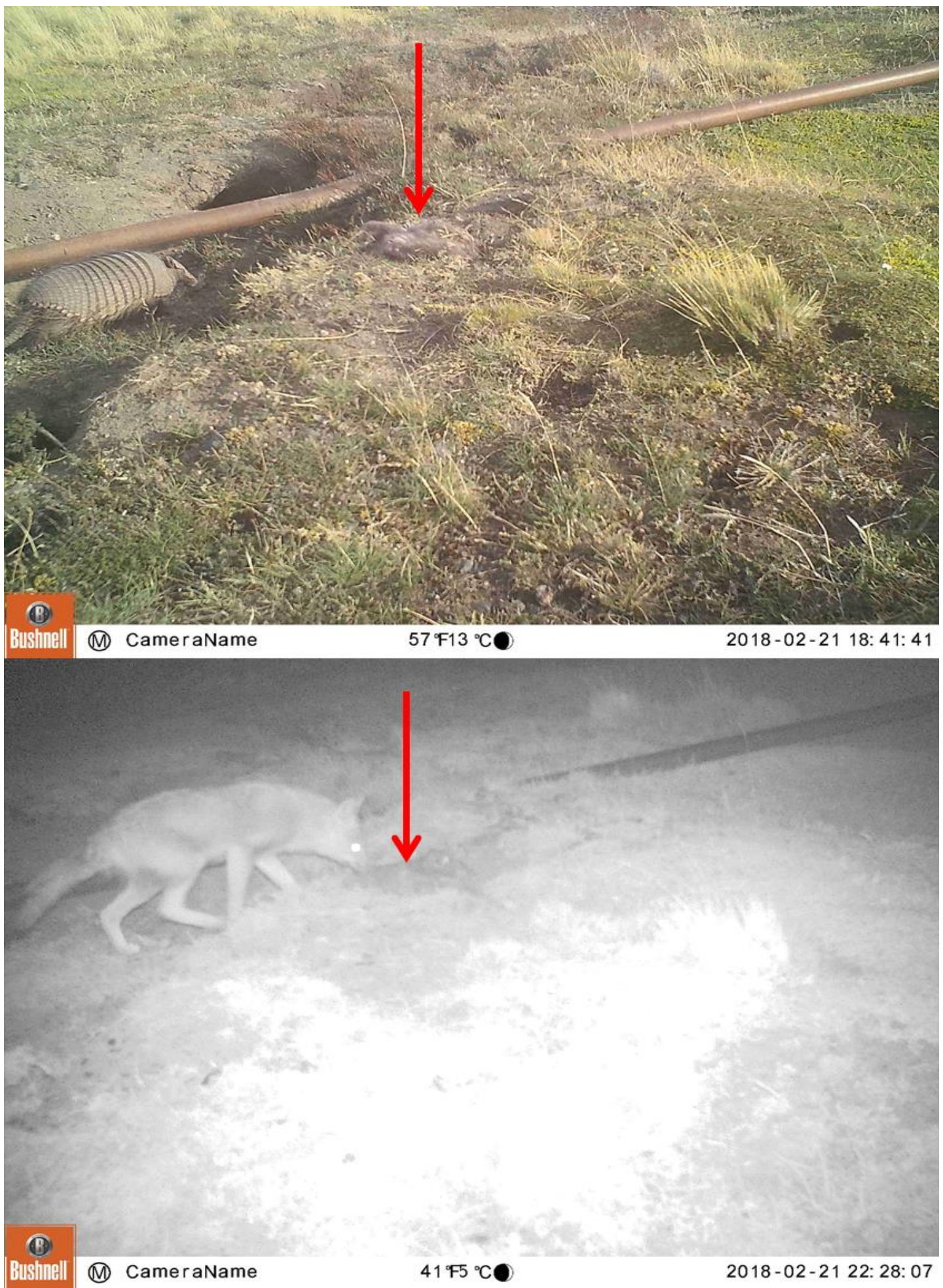

Figura 4.6. Imágenes de un nido artificial N\#7. A) Ejemplar de peludo en las inmediaciones del nido artificial. B) El mismo nido al momento de ser depredado unas horas más tarde por un ejemplar de zorro gris. Las flechas rojas indican la posición del nido. 


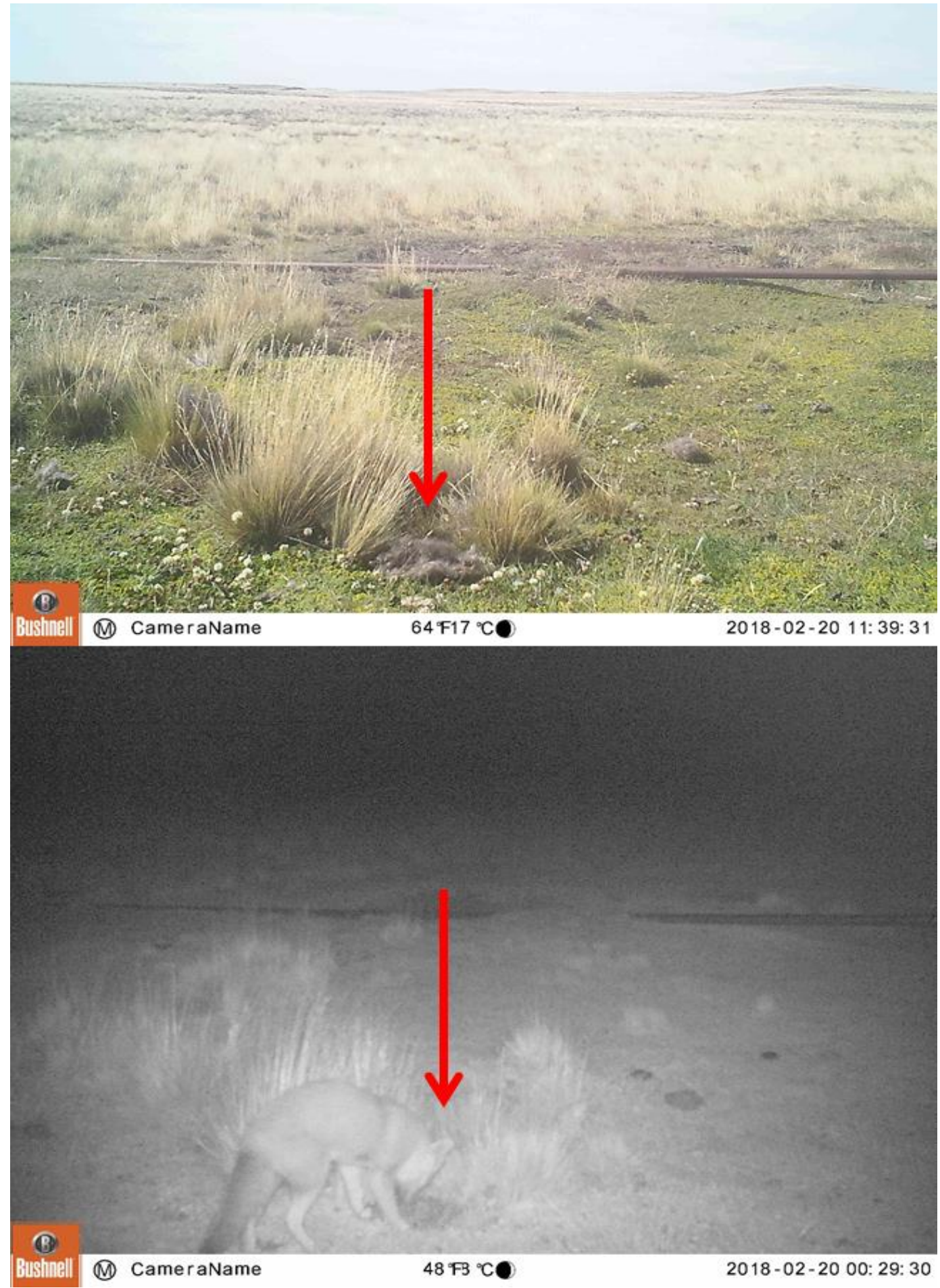

Figura 4.7. Imágenes del nido artificia N\# 5. Arriba durante el día. Abajo durante la noche, donde se observa un ejemplar de zorro gris depredando el nido. Las flechas rojas indican la posición del nido 


\subsection{DISCUSIÓN Y CONCLUSIONES}

La composición de la dieta de C. villosus en la Isla Grande de Tierra del Fuego presenta una diversidad menor en comparación con su distribución nativa. En un nuevo ambiente, los depredadores generalistas tienden a cambiar su dieta incorporando aquellos ítems presa de mayor abundancia (Pyke et al., 1977). Si bien la cantidad de muestras utilizadas para Tierra del Fuego fue menor, limita la posibilidad de encontrar algunos items presa y por lo tanto, obtener un menor valor de diversidad, los pocos estudios que existen acerca de la diversidad de artrópodos en Tierra del Fuego, aseguran que la riqueza específica de invertebrados terrestres, principalmente de insectos, disminuye con la latitud y que la Isla Grande de Tierra del Fuego, debido a sus condiciones climáticas extremas, representa un ambiente poco favorable para varias especies de artrópodos (Peck, 1986; Posadas, 2012). Si bien no hay registros sobre la disponibilidad y abundancia de los diferentes ítems, es posible que por esta razón algunos ítems con frecuencia de ocurrencia menor al 50\% en el continente, como ser Scarabaeidae (adultos y larvas), lepidópteros y larvas de diptertos, aumenten notablemente la frecuencia de ocurrencia en Tierra del Fuego. Así mismo, ítems bajo la categoría de Terciarios en el continente, como ser larvas de Sacarabaeidae y Restos Vegetales, en Tierra del Fuego suben a la categoría Primario y Secundario, respectivamente, lo que refleja un mayor consumo de aquellos ítems disponibles en la Isla Grande.

Estudios sobre ecología trófica del peludo en su distribución nativa tanto en Patagonia (ver Arriagada et al. 2017; esta Tesis) como para el resto de su distribución (ver Gallo et al., 2019) muestran que los ítems principales en la dieta de este armadillo son "carroña" y "restos vegetales". Sin embargo, los ítmes considerados dominantes en la población invasora de Tierra del Fuego son "restos vegetales" (principalemente raíces y tubérculos) y "larvas de Scarabaeidae". Ambos ítemms son hipógeos, es decir, se encuentran bajo la superficie del suelo. Particularmente las larvas de Sacarabaeidae se encuentran entre los $15 \mathrm{~cm}$ y $30 \mathrm{~cm}$ de profundidad (Peck, 1986) y son abundantes en suelos con alto contenido de materia orgánica, como los suelos húmicos (Rodríguez et al., 
2004), que además coincide con el tipo de suelo asociado a la presencia de C. villosus en la Isla Grande de Tierra del Fuego (Ver Capítulo 2).

Los restos de tejidos de vertebrados (carroña) es un ítem presa muy común en $C$. villosus (Redford, 1985; Arriagada et al., 2017; Gallo et al., 2019). En la Isla Grande de Tierra del Fuego, el vertebrado más abundante es la oveja, destinado a la producción de lana. La mayor parte de los lotes de pastoreo de ganado ovino en la provincia de Tierra del Fuego se dividen en sitios de veranada, ubicados principalmente al Oeste (hacia el límite con Chile), y lotes de invernada, ubicados hacia el Este (Boletín Estadístico Agropecuario Tierra del Fuego, 2015). La colecta de individuos de C. villosus fue durante la temporada estival y en la zona de San Sebastián, al Este de la Isla Grande. Éste puede ser el motivo por le el cual el item "Carroña" se vió representado en las muestras a partir de las cuales se determinó la dieta del peludo en Tierra del Fuego, ya que los restos de ganado en la zona muestreada no serían un ítem disponible en grandes cantidades, como sí lo es en el continente o durante la época invernal en este sector de Tierra del Fuego. Una profundización del estudio de dieta para explorar variaciones en la dieta en relación a la disponibilidad estacional de otros recursos (e.g. en casos de superposición espaciotemporal con ovejas) podrían confirmar o descartar lo planteado en este capítulo.

Los resultados del análisis de depredación de $C$. villosus sobre aves que nidifican en el suelo a partir del monitoreo de nidos artificiales en áreas de alta actividad de peludos no apoyan la hipótesis de que los peludos de Tierra del Fuego depredan activamente sobre nidos de aves nidificantes de suelo. Aunque los nidos fueron colocados en zonas cercanas a cuevas activas de peludos, solo uno de los nidos fue visitado por $C$. villosus y sin mostrar un comportamiento depredatorio sobre los huevos, mientras que cinco de los ocho nidos artificiales colocados fueron depredados por zorro gris. Esto puede deberse a que el zorro gris resulta ser un carnívoro depredador más especializado, de mayor movilidad y capacidad visual que el peludo. La alta densidad de zorros grises en la zona podría ocasionar que los huevos sean un recurso con una disponibilidad aparente baja para el armadillo invasor. Quizás por esta razon los huevos de aves fueron categorizados como Ocasionales en el continente. Cossa et al. (datos no publicados) describieron que la 
tasa de supervivencia de los nidos construidos en el suelo en Santa Cruz y Tierra de Fuego cae por encima del 50\% luego de los 10 días, debido principalmente a la depredación de zorros grises.

Si bien no se observó un comportamiento depredatorio por parte del peludo sobre los nidos artificiales ni tampoco restos de vertebrados en su dieta, es probable que, ante la posibilidad, este armadillo deprede sobre huevos de aves (Figura 4.8; Gallo et al., 2019) y vertebrados pequeños (Arriagada et al., 2017; Gallo et al., 2019).

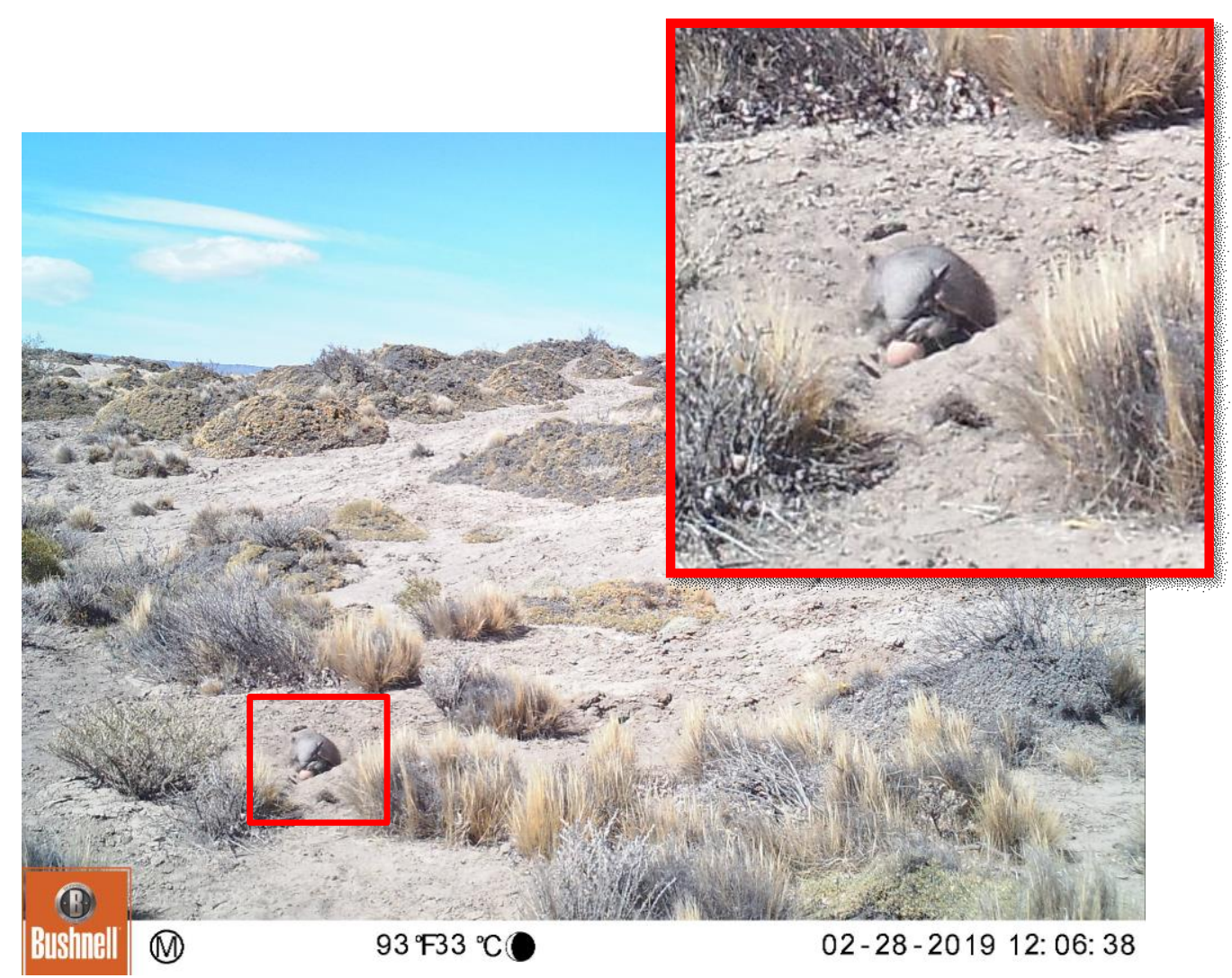

Figura 4.8. Chaetophractus villosus depredando sobre un huevo de nido artificial en Santa Cruz (Cossa et al., datos no publicados). Fotografía de Cossa N.

Si bien los resultados de este estudio no demuestran que el peludo deprede sobre huevos de aves o sobre pequeños vertebrados, es necesario considerar que la presencia 
de $C$. villosus en la Isla Grande de Tierra del fuego como un mamífero omivoro-carnivoro generalista, sumaría una nueva especie al ensamble de mamíferos exóticos depredadores presentes en la zona, como lo son el visón americano (Neovison vison), el zorro gris (Lycalopex gymnocercus) y los perros asilvestrados (Canis familiaris), complicando aún más la situación poblacional de las especies nativas de la Isla.

\section{BIBLIOGRAFÍA}

ABBA, A.M.,D.E. UdRIZAR, AND S.F. VIZCAínO. 2005. Distribution and use of burrows and tunnels of Chaetophractus villosus ( Mammalia, Xenarthra) in the Eastern Argentinean pampas. Acta theriologica 50:115-124.

ARBeiter, S., AND E. Franke. 2018. Predation risk of artificial ground nests in managed floodplain meadows. Acta Oecologica 86:17-22.

ARRIAGAdA, A. ET AL. 2017. Hábitos alimenticios de poblaciones periféricas de Zaedyus pichiy y Chaetophractus villosus (Cingulata, Chlamyphoridae) en la Patagonia chilena. Iheringia Serie Zoologia 107:e2017103.

CAnevarI, P. 1996. The austral geese (Chloephaga spp.) of Southern Argentina and Chile: a review of its current status. Gibier faune sauvage 13:355-366.

D.G. de E.Y.C. 2015. Boletín estadístico agropecuario. Ushuaia, Tierra del Fuego.

Cossa, N.A., L. FAsola, I. Roesler, AND J.C. ReboredA. 2017. Ruddy-headed Goose Chloephaga rubidiceps: former plague and present protected species on the edge of extinction. Bird Conservation International 27:269-281.

DICKMAN, C.R. 1996. Impact of exotic generalist predators on the native fauna of Australia. Wildlife Biology 2:185-195.

GallinA-TeSSARo, S. 2011. Técnicas para conocer la dieta. P. 377 in Manual de técnicas para el estudio de la fauna. Volúmen I. (S. Gallina \& C. López-González, eds.). Universidad Autónoma de Querétaro-Instituto de Ecología, Querétaro, México.

Gallo, J., L. FAsola, And A. AbBA. 2019. Armadillos as pest controllers? Food habits of five armadillos in Argentina. Mastozoologia Neotropical.

GroombridGE, B. 1992. Global biodiversity: status of the earth's living resources. Chapman \& Hall, London. 
Grosman, F., G. González, P. SAnzano, And D. Agüería. 2002. Alimentación, nichos tróficos y competencia interespecífica de peces de la laguna de Monte , Argentina. Pp. 129-140 in.

Grosman, F., P. Sanzano, D. Agüeria, G. González, and S. Sergueña. 2001. Ecología reproductiva, edad, crecimiento, condición y alimentación del pejerrey (Odontesthes bonariensis) en un ambiente del SO de la provincia de Buenos Aires, Argentina. Revista AquaTIC 12:20.

Grosman, M. F. 1995. Variaciones estacionales en la dieta del pejerrey (Odonthes bonariensis). Revista de la Asociación de Ciencias Natirales del Litoral 26:9-18.

HeRnÁNDEZ, C., S. SADE, AND J. Rau. 2017. Dieta del jabalí (Sus scrofa), invasor biológico reciente del Parque Nacional Puyehue, sur de Chile. Mastozoología Neotropical 24:467-473.

JOHNSON, D. H. 1980. The comparison of usage and availability measurements for evaluating resource. Ecology 61:65-71.

KAMENOVA, S. et al. 2017. Invasions toolkit: current methods for tracking the spread and impact of invasive species. Advances in Ecological Research 56: 85-182

KLUG, P., L. L. Wolfenbarger, AND J. P. McCARTy. 2009. The nest predator community of grassland birds responds to agroecosystem habitat at multiple scales. Ecography 32:973-982.

LAVILLA, E., E. RICHARD, AND G. SCROCCHI. 2000. Categorización de los anfibios y reptiles de la República Argentina. Asociación Herpetológica Argentina. San Miguel de Tucumán.

Lyons, T. P., J. R. Miller, D. M. Debinski, ANd D. M. Engle. 2015. Predator identity influences the effect of habitat mangement on nest predation. Ecological Applications 25:In Press.

MANCINI, M., AND F. Grosman. 1998. Aspectos poblacionales del pejerrey Odontesthes bonariensis en el Embalse Río Tercero, Córdoba. Natura Neotropicalis. .

MARTIN, T. 1993. Nest Predation and Nest Sites. BioScience 43:523-532.

Mcdonough, C. M., AND W. J. Loughry. 2008. Behavioral ecology of armadillos. The Biology of the Xenarthra 26:281-293.

PECK, S. B. 1986. Adult habits, larval morphology, and phylogenetic placement of Taurocerastes patagonicus Philippi ( Scarabaeidae : Geotrupinae ). Canadian Journal of Zoology 25:329-332. 
POSADAS, P. 2012. Species composition and geographic distribution of Fuegian Curculionidae (Coleoptera: Curculionoidea). Zootaxa 36:1-36.

Pyke, G. H., H. R. Pulliam, AND E. L. Charnov. 1977. Optimal foraging: a selective review of theory and tests. The Quarterly Review of Biology 52:137-154.

REDFORD, K. H. 1985. Food habits of armadillos (XENARTHRA: DASYPODIDAE). Pp. 429-437 in The evolution and ecology of armadillos, sloths and vermilinguas (G. . Montgomery, ed.). Smithsonian Institution Press, Washington \& London.

Rodríguez, M., A. France, And M. Gerding. 2004. Evaluación de dos cepas del hongo Metarhizium anisopliae var. Anisopliae (metsh.) para el control de larvas de gusano blanco Hylamorpha legans Burm. (COLEOPTERA: SCARABAEIDAE). Agricultura Técnica 64:17-24.

Ruffino, L., J. C. Russell, B. PisAnu, S. CAut, ANd E. Vidal. 2011. Low individual-level dietary plasticity in an island-invasive generalist forager. Population Ecology 53:535-548.

Salo, P., E. Korpimaki, P. B. Banks, M. Nordstrom, AND C. R. Dickman. 2007. Alien predators are more dangerous than native predators to prey populations. Proceedings of the Royal Society B: Biological Sciences 274:1237-1243.

SÁNCHEZ, J., AND M. S. LizARRALDE. 2019. Ctenomys magellanicus. Categorización 2019 de los mamíferos de Argentina según su riesgo de extinción. Lista Roja de los mamíferos de Argentina. (SAyDS-SAREM, ed.). SAyDS-SAREM.

SiH, A., AND B. ChrISTENSEN. 2001. Optimal diet theory: When does it work, and when and why does it fail? Animal Behaviour 61:379-390.

STEHR, F. W. 1987. Inmature Insects. Vol I. Kendall/Hunt publishing company, Dubuque, Iowa, USA.

STEHR, F. W. 1991. Inmature Insects.Vol II. Kendall/Hunt publishing company, Dubuque, Iowa, USA. 

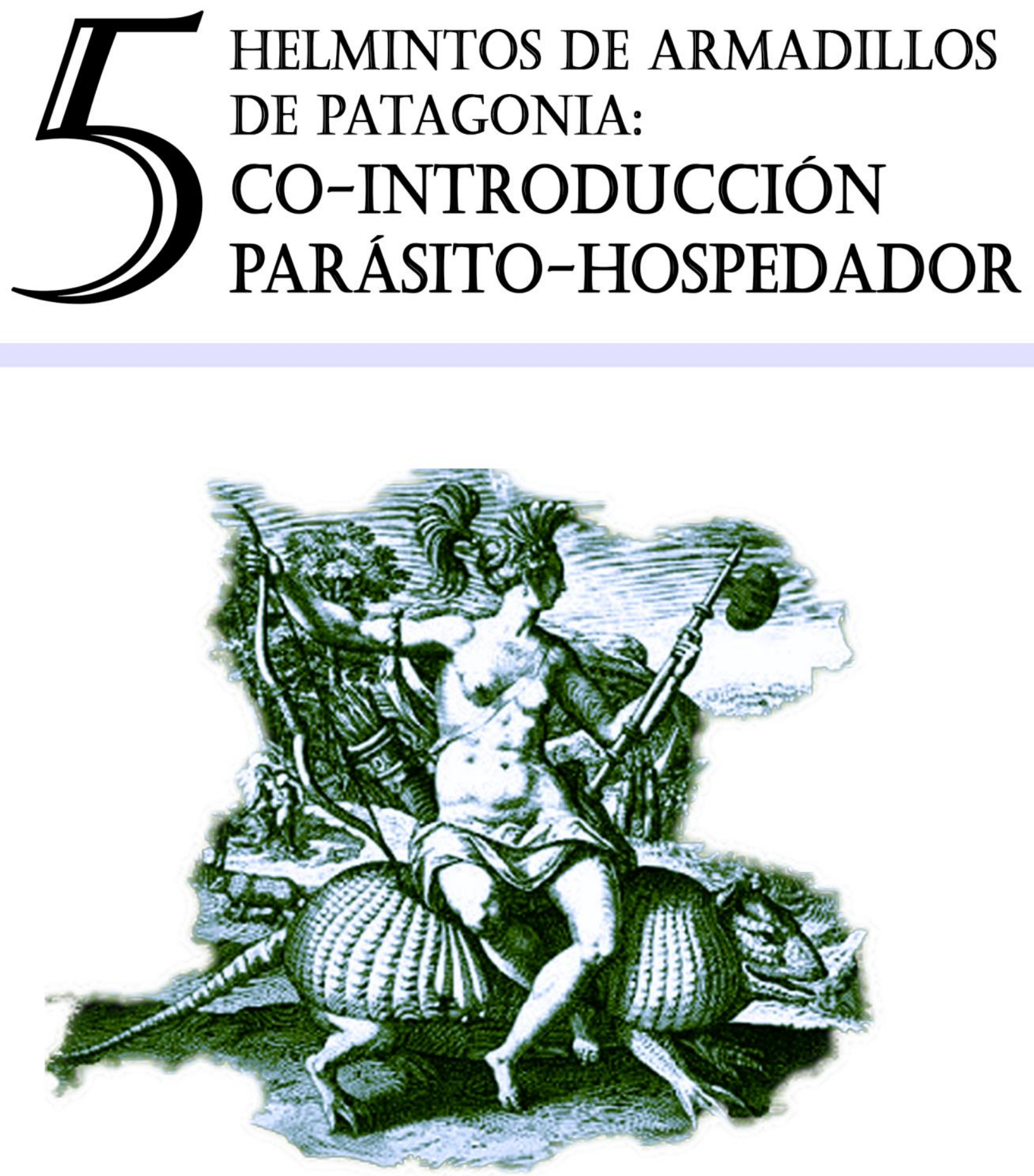

Marten de Vos y Adriaen Collaert. Las alegorías de los continentes, Amsterdam. 1600

"Hijuay pucha quirquincho Carita de piedra poma, Yo le 'i decir al zorrito Que ande te vea te coma" 


\subsection{INTRODUCCIÓN}

La diversidad de helmintos responde principalmente a la exposición del hospedador a determinadas especies parásitas, lo que está asociado a las características morfológicas del hospedador, sus hábitos y a los factores ambientales de su área de distribución (Krasnov et al., 2004; Luque y Poulin, 2008). Sin embargo, Bordes et al. (2010) aseguran que la exposición del hospedador no es el único factor que afecta la diversidad parasitaria. La riqueza específica también resulta de los hábitos del parásito, de su ecología y de las defensas del hospedador (Bush et al., 1997). En este sentido, numerosos estudios reconocen que la latitud de la distribución del hospedador es determinante de la riqueza específica de helmintos (Poulin, 1995; Krasnov et al., 2004; Nunn et al., 2005; Lindenfors et al., 2007; Ezquiagaet al., 2016; Poulin y Leung, 2011). Es de esperar que a mayores latitudes disminuya la diversidad de especies parásitas, principalmente nematodes, debido a una alta mortalidad de estadios intermedios y a la falta de hospedadores intermediarios, debido a condiciones climáticas extremas (Nunn et al., 2005; Bordes et al., 2010; Ezquiaga et al., 2016; Poulin and Leung, 2011).

Por otra parte, los patrones geográficos del hospedador también determinan la comunidad parasitaria. La similitud entre comunidades parásitas decrece en función de la distancia (Poulin and Krasnov, 2010a). En este contexto, la colonización de nuevas regiones por parte de un hospedador, ya sea por dispersión natural o por introducción, produce pérdida de especies parásitas, transferencias o adquisición de nuevas especies (Lafferty et al., 2010). En relación a esto, Lymbery et al. (2014) definen dos términos importantes en la biología de invasiones: 1) se habla de co-introducción parásitohospedador cuando un parásito junto a su hospedador logran establecerse en una nueva región, y 2) co-invasión parásito-hospedador cuando un parásito logra colonizar nuevas especies hospedadoras nativas en la región introducida.

Chaetophractus villosus (peludo) y Zaedyus pichiy (piche) son las únicas dos especies de armadillos que actualmente habitan Patagonia (Abba et al., 2014). Ambas especies son relativamente similares en sus aspectos generales, la mayor diferencia es su tamaño ya que C. villosus pesa en promedio 3,5 kg y Z. pichiy $1 \mathrm{~kg}$ (Superina and Abba, 
2018). Como fue nombrado en capítulos anteriores (ver Capítulos 1 y 2) la distribución del peludo abarca prácticamente toda la Argentina, pero el piche está presente en la región centro-oeste del país (La Rioja, San Juan, Mendoza, San Luis, Córdoba, La Pampa), sur de Buenos Aires y toda la Patagonia excepto Tierra del Fuego (Superina et al., 2019). El piche habita en regiones áridas o semi-áridas del monte (26\% de su distribución, ver Abba et al., 2012) aunque principalmente su distribución se encuentra en la estepa patagónica (40\% de su distribución, ver Abba et al., 2012). Su dieta es similar a la del peludo e incluye invertebrados, restos vegetales y pequeños vertebrados, por lo que es considerado carnívoro-omnívoro (Gallo et al., 2019).

A pesar de que estas dos especies de armadillos comparten un amplio rango de sus distribuciones, la cronología y proceso de colonización de Patagonia fue diferente. Según Abba et al. (2014) Z. pichiy ya era una especie común y ampliamente distribuida en Patagonia durante el Holoceno (excepto en la región al sur del Río Santa Cruz). Sin embargo, C. villosus sería una especie relativamente reciente en Patagonia (Abba et al., 2014). Esta especie habría dispersado desde la región pampeana, considerada como la región de origen o núcleo (Poljak et al., 2010), hacia el sur del Río Chubut hace aproximadamente 100 años, facilitada en parte por actividades humanas como construcción de caminos, puentes y el aumento de la producción agropecuaria (Abba et al., 2014). La tasa de expansión en Patagonia estimada fue de $13 \mathrm{~km} / \mathrm{año} \mathrm{(Abba} \mathrm{et} \mathrm{al.,}$ 2014), similar a la tasa calculada para Tierra del Fuego (10,9 km/año; ver Capítulo 2). Por lo tanto, la presencia del peludo tanto en Patagonia continental como en la Isla Grande de Tierra del Fuego es relativamente reciente (en escala evolutiva).

Los estudios acerca de la helmintofauna de estos dos armadillos en Patagonia son escasos (Ezquiaga et al., 2009, 2016, 2017). Sin embargo, la fauna parasitaria para regiones del centro-norte del país está muy bien documentada (Navone, 1990; Ribicich et al., 2010; Ezquiaga et al., 2012; Ezquiaga, 2013; Ezquiaga y Navone, 2014; Rios, 2020). Ezquiaga (2013) describió 11 especies de helmintos en ejemplares de C. villosus, todos registros de localidades más al norte de la provincia del Chubut. El último estudio sobre el peludo en Patagonia lo realizaron Ezquiaga et al. (2016) a partir de muestras colectadas 
entre 2002 y 2006 (ver Poljak et al., 2007) y en el mismo se describe una drástica disminución de la riqueza parasitaria en la población introducida de Tierra del Fuego, la que presentó una sola especie parásita, Trichohelix tuberculata (Parona y Stossich, 1902) (Trichostrongylina: Molineidae), respecto a las 11 especies presentes en la región núcleo (Ezquiaga et al., 2016). Para la distribución norte de Zaedyus pichiy se conocen 12 especies de helmintos. Primero, Ezquiaga et al. (2009), mediante técnicas coproparasitológicas, describen huevos de 5 helmintos para la parte norte de la estepa patagónica, en Mendoza. Luego, Ezquiaga (2013), mediante el análisis del tracto digestivo, encuentra 11 especies de helmintos, incluyendo los hallados en el estudio anterior (Ezquiaga et al. 2009) excepto por Trichuris sp. Además del estudio realizado por Ezquiaga et al. (2016) en Tierra del Fuego, no existe otro estudio sobre ecología de helmintos de armadillos de Patagonia centro-sur (Chubut, Santa Cruz y Tierra del Fuego).

El objetivo de este capítulo es describir la helmintofauna de las dos especies de armadillos de Patagonia y analizar las variaciones en la diversidad de helmintos de $Z$. pichiy y C. villosus. Para ello se realizaron comparaciones entre las poblaciones de Patagonia sur (Chubut, Santa Cruz y Tierra del Fuego) y las poblaciones estudiadas al norte de la provincia de Chubut por Ezquiaga et al. (2009) y Ezquiaga (2013) para ambos hospedadores.

\subsection{MATERIALES Y MÉTODOS}

\subsection{1 Área de estudio}

Durante las temporadas de verano de 2017, 2018 y 2019 se realizaron campañas en la región Patagónica, en las provincias de Chubut, Santa Cruz y Tierra del Fuego, para la obtención de muestras.

El área de estudio corresponde a la región biogeográfica Estepa Patagónica, que ocupa gran parte de Chubut, casi toda la provincia de Santa Cruz y el norte de Tierra del Fuego (Cabrera, 1971). Presenta un clima con características de semidesierto, frío, ventoso y seco, con una precipitación media anual inferior a $250 \mathrm{~mm}$ aumentando hacia Tierra del Fuego. La vegetación predominante es de matorrales achaparrados y arbustos 
bajos, y en menor proporción aparecen especies herbáceas (Matteucci, 2011). Se observan mesetas y montañas bajas con suelos esqueléticos arenosos-pedregosos (Cabrera, 1971). Por su parte, la estepa de Tierra del Fuego presenta características similares con mayor cantidad de precipitaciones y una fisonomía más heterogénea (Ver Capítulo 1). Para más información ver Capítulo 1.

\subsubsection{Recolección del material}

Durante la temporada de verano de 2019 se recorrieron rutas principales y caminos secundarios de Chubut y Santa Cruz, en busca de armadillos atropellados, colectando un total de 23 ejemplares de Chaetophractus villosus y 14 de Zaedyus pichiy. Para la provincia de Tierra del Fuego, se realizaron dos campañas durante la temporada estival de los años 2017 y 2018. En este caso se capturaron 12 C. villosus y se sacrificaron utilizando un eutanásico de vía circulatoria (ver Capítulo 4; Resolución S.A.D.SyC.C.064/2014, Provincia de Tierra del Fuego). Se recolectaron un total de 49 hospedadores de diferentes localidades para toda Patagonia (Figura 5.1). 


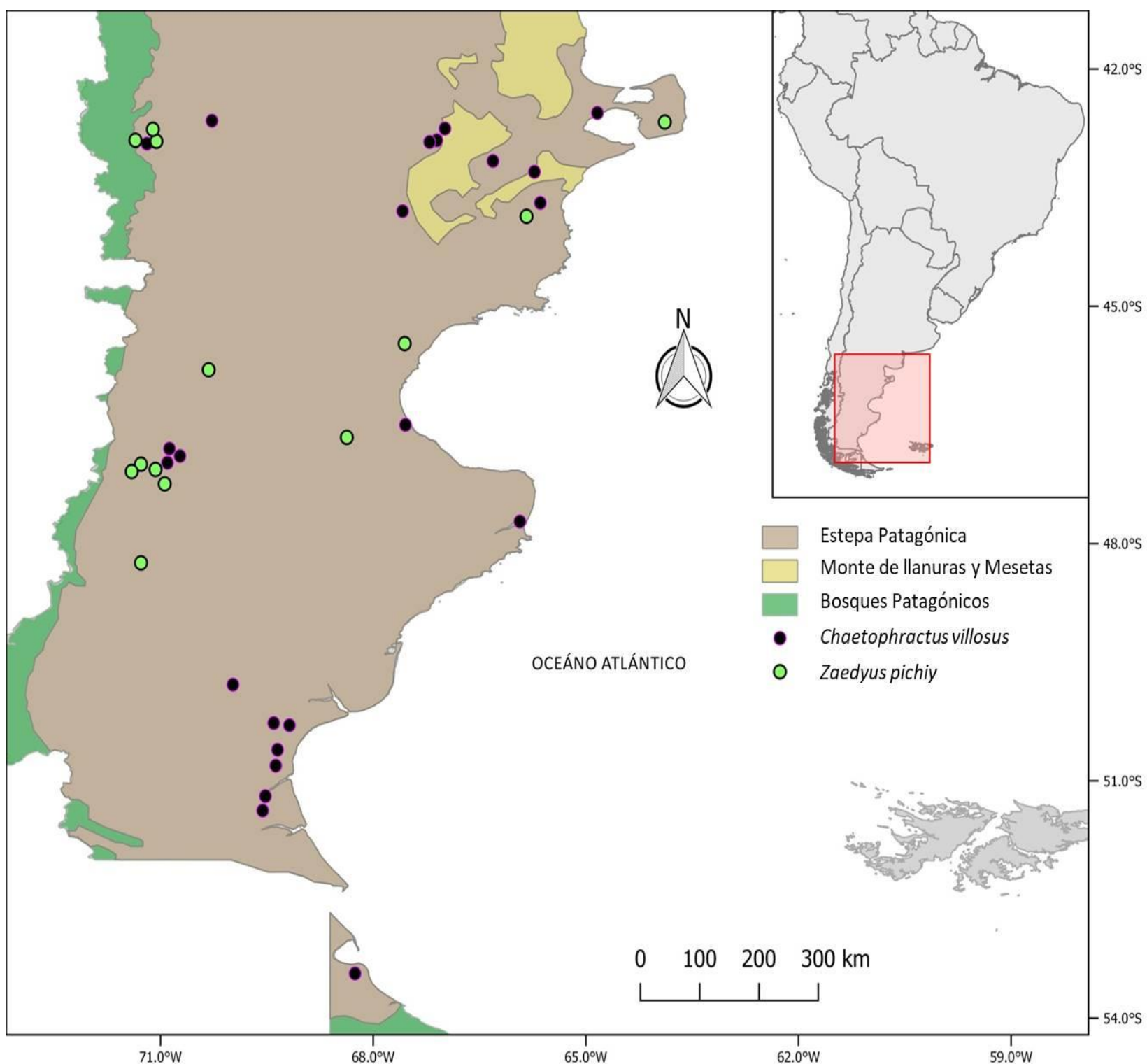

Figura 5.1 Eco-regiones patagónicas argentinas indicando la ubicación y especies hospedadoras muestreadas.

Para la obtención del tracto digestivo, se colocó al animal en posición decúbito dorsal y se realizó un corte ventral longitudinal. El tracto digestivo completo fue extraído y fijado en una solución de formaldehido 10\% (Figura 5.2). 
Tanto para la eutanasia como para el manejo del material se siguieron las normas de ética y seguridad vigentes (Sikes et al., y ASM, 2016).

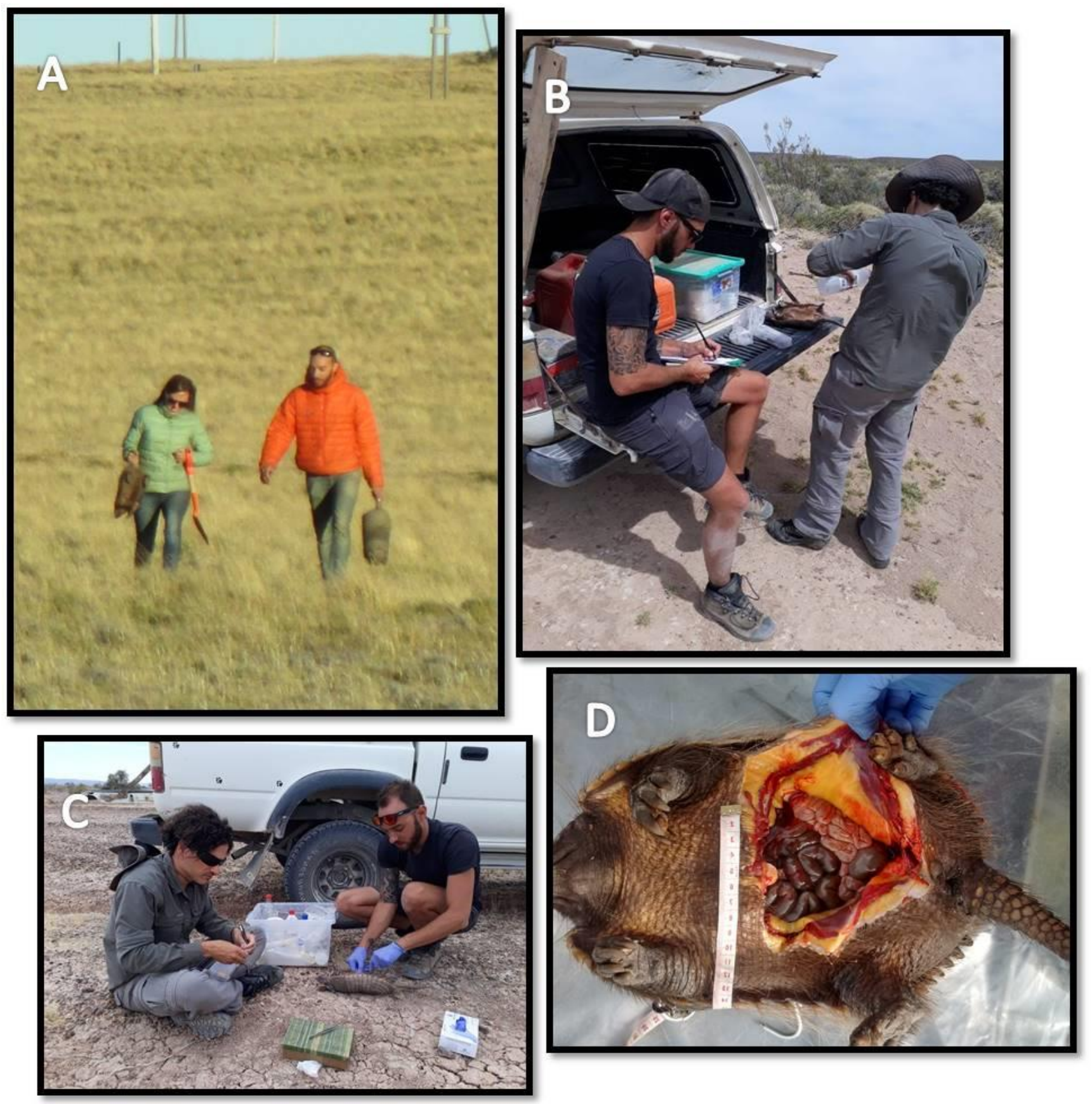

Figura 5.2 Imágenes de la recolección de hospedadores en el campo. A) Colecta manual de C. villosus en Tierra del Fuego. B) Toma de datos a campo. C y D) Evisceración de ejemplar de C. villosus. 


\subsubsection{Trabajo de laboratorio y análisis de estadísticos}

Se revisaron las vísceras y fueron extraídos todos los helmintos. Para ello se realizó un corte longitudinal en todo el tracto digestivo revisando su contenido bajo lupa binocular Lancet 10x-40x. Para su identificación, se observaron los helmintos bajo lupa binocular, posteriormente, se transparentaron con alcohol glicerinado y se realizaron preparados transitorios para observar bajo microscopio óptico. Los nematodes fueron identificados utilizando las claves dicotómicas de Anderson et al. (2009) y bibliografía específica. Para la identificación de cestodes se consultó a Khalil et al. (1994) y se realizaron consultas a un especialista (M. C. Ezquiaga). El material será depositado en la colección helmintológica del Museo de La Plata.

Una vez identificados los helmintos, se contabilizaron y se realizaron los siguientes análisis ecológicos (Bush et al., 1997):

1. Análisis de la población componente: se define como todos los individuos de una especie parásita que ocurren en una población hospedadora determinada. Para su análisis se calcularon los siguientes parámetros:

$\rightarrow$ Prevalencia: número de ejemplares parasitados/número de hospedadores examinados x 100 (Bush et al., 1997)

$\rightarrow$ Abundancia Media: número de especímenes de una especie parásita/número de hospedadores examinados (Bush et al., 1997)

$\rightarrow$ Intensidad Media: número de especímenes de una especie parásita/número de hospedadores parasitados (Bush et al., 1997).

Las prevalencias de aquellos parásitos compartidos entre hospedadores se analizaron mediante una prueba de independencia de Chi-cuadrado y una prueba de Fisher ( $\alpha=$ $0,05)$.

2. Análisis de la comunidad componente: se define como comunidad componente a todas las poblaciones parásitas que explotan una población hospedadora en un tiempo dado. Para ello se calcularon los siguientes índices:

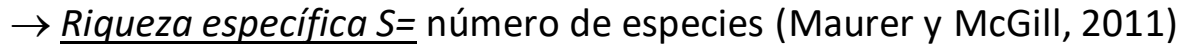


$\rightarrow$ Diversidad específica: Índice de diversidad de Shannon-Wiener: $\mathrm{H}^{\prime}=-\sum \mathrm{p}_{\mathrm{i}} \ln \mathrm{p}_{\mathrm{i}}$ Donde $\mathrm{p}_{\mathrm{i}}$ : proporción de cada especie respecto al total de especies parásitas (Maurer y McGill, 2011).

Estructura de la comunidad parasitaria: Se utilizaron los valores de prevalencia e intensidad media para aplicar los conceptos de especies centrales, secundarias y satélites desarrollados por Bush y Holmes (1986). Las especies centrales son aquellas comunes y numerosas en la mayoría de las infracomunidades, mientras que las especies satélite se encuentran en pocas infracomunidades y en bajo número. Para poder distinguir entre especies parásitas Centrales, Secundarias y Satélites se deben cumplir dos supuestos. Por un lado la distribución de las frecuencias de las prevalencias de las especies parásitas debe ser bimodal o trimodal. Por otro lado, la correlación entre la prevalencia (para representar la dispersión regional) y la intensidad media (para representar la abundancia local) debe ser positiva. En el caso de una distribución trimodal se puede distinguir entre especies centrales, secundarias y satélites, pero si la distribución de frecuencias es bimodal, se podrá distinguir solo entre especies Centrales y Satélites (Holmes, 1987).

A su vez, se aplicó el siguiente índice de Importancia (I) desarrollado por Thul et al. (1985):

$$
I=(M j) \frac{A j B j}{\sum_{i-1}^{6} A j B j}
$$

Siendo $\mathrm{Mj}$ el factor de madurez, el cual adquiere valor igual a 1 si se encontró al menos un ejemplar del helminto $j$ maduro sexualmente o igual a cero si solo se encontraron ejemplares inmaduros (larvas). $A j$ es el número de individuos de la especie parásita $j$, y $B j$ es el número de hospedadores en los que aparece la especie parásita $j$.

Este índice permite explicar la contribución de cada especie parásita a la comunidad y distingue entre cuatro categorías: Especie dominante $(I \geq 1)$, especie co-dominate $(0,01 \leq 1$ $\leq 1)$, especie subordinada $(0,0<I<0.01)$ y especie pionera no satisfactoria $(I=0)$. 
Para realizar los análisis detallados anteriormente se distinguieron las siguientes poblaciones para cada hospedador:

Zaedyus pichiy:

- Población Norte: Incluye localidades de las provincias de Mendoza, La Pampa y Buenos Aires muestreadas por Ezquiaga et al. (2009) y Ezquiaga et al. (2013).

- Población Patagonia Sur: Localidades de las provincias de Chubut y Santa Cruz muestreadas en esta Tesis.

Chaetophractus villosus:

- Población Núcleo: Incluye las localidades de la región Pampeana muestreadas por Ezquiaga et al. (2013).

- Población Patagonia Sur: Muestras de Chubut y Santa Cruz analizadas en esta Tesis

- $\quad$ Población TdF 2002-2006: Incluye la población de Tierra del Fuego analizada por Ezquiaga et al. (2016), cuyas muestras fueron recolectadas entre 20022006 (Poljak et al., 2010).

- $\quad$ Población TdF actual: Muestras de Tierra del Fuego analizadas en esta Tesis.

Se realizó un análisis de ecología parasitaria general para ambos hospedadores en Patagonia y luego, con fines prácticos, se dividieron las poblaciones de hospedadores y se realizaron las siguientes comparaciones:

- Z. pichiy Población Norte vs. Z. pichiy Patagonia Sur.

- C. villosus Población núcleo vs. C. villosus Patagonia Sur.

-C. villosus TdF 2002-2006 vs. C. villosus TdF Actual.

Se compararon valores de prevalencia de aquellos parásitos compartidos entre poblaciones de un mismo hospedador y se analizaron las diferencias mediante una prueba de independencia de Chi-cuadrado ( $\alpha=0,05$; Morales y Pino, 1987). Se excluyen del análisis comparativo, los huevos de helmintos descriptos por Ezquiaga et al. (2009), ya que se trata de metodologías muy diferentes a las realizadas en esta tesis. 


\subsection{RESULTADOS}

\subsubsection{Análisis de la población y comunidad componente de helmintos para ambos hospedadores en Patagonia}

De los 49 hospedadores analizados, 48 estuvieron parasitados, por lo tanto se obtuvo en prevalencia total de $97,9 \%$. Las prevalencias totales de parasitismo por especie hospedadora fueron del $100 \%$ para C. villosus y $92,8 \%$ para $Z$. pichiy. Se contabilizaron un total de 7559 parásitos pertenecientes a dos grandes grupos: nematodes (7546) y cestodes (13), los cuales se encontraron en el estómago, intestino delgado, ciego e intestino grueso. La riqueza específica total fue de 8 taxones de helmintos para toda Patagonia, de los cuales 7 corresponden a nematodes y 1 a cestodes. Ambas especies de hospedadores ( $C$. villosus y $Z$. pichiy) obtuvieron una riqueza específica $S=6$. El índice de diversidad de Shanon $\left(H^{\prime}\right)$ fue de 0,954 para C. villosus y 0,755 para Z. pichiy.

En la Tabla 5.1 se muestran todas las especies de helmintos hallados para Patagonia con sus parámetros poblacionales (Prevalencia, Intensidad Media y Abundancia Media). Aspidodera fasciata (Schneider, 1866) (Ascaridida:Aspidoderidae), A. scoleciformis (Diesing, 1851) (Ascaridida:Aspidoderidae), Pterygodermatites argentinensis (Ezquiaga, Rios, Abba y Navone, 2017) (Spirurida:Rictulariidae) y Physalopteridae (Spirurida: Physalopteridae) (Railliet, 1893) estuvieron presentes en ambos hospedadores con prevalencias moderadas a bajas $(<50 \%)$. Se observaron diferencias significativas en las prevalencias para una especies parasita compartidas por ambos hospedadores, Aspidodera scoleciformis $\left(X^{2}=10,7 ; p=<0,001 ; g l=1\right)$. Los valores más altos de Intensidad Media y Abundancia Media en $C$. villosus fueron para los helmintos Trichohelix tuberculata, Aspidodera fasciata y A. scoleciformis, mientras que de Z. pichiy fueron para ambas especies de Aspidodera. 
Tabla 5.1. Parámetros poblacionales de los helmintos parásitos de Chaetophractus villosus y Zaedyus pichiy en Patagonia centro-austral. Riqueza (S), Prevalencia (\%), Intensidad Media (IM) y Abundancia Media (AM). Los estadios larvarios se indican con la letra L.

\begin{tabular}{|c|c|c|c|c|c|c|c|c|c|c|}
\hline \multirow{3}{*}{ Parásito } & \multicolumn{6}{|c|}{ Chaetophractus villosus } & \multirow{2}{*}{\multicolumn{3}{|c|}{$\begin{array}{l}\text { Zaedyus pichiy } \\
\text { Patagonia Sur }\end{array}$}} & \multirow{3}{*}{$\begin{array}{c}\text { Sitio de } \\
\text { infección }\end{array}$} \\
\hline & \multicolumn{3}{|c|}{ Patagonia Sur } & \multicolumn{3}{|c|}{ TdF actual } & & & & \\
\hline & $\mathrm{P}(\%)$ & IM & AM & $\mathrm{P}(\%)$ & IM & AM & $\mathbf{P}(\%)$ & IM & AM & \\
\hline Aspidodera fasciata & 39,1 & 138,7 & 54,3 & - & - & - & 50,0 & 163,1 & 96,6 & C. + I.G. \\
\hline Aspidodera scoleciformis & 17,4 & 117,8 & 20,5 & - & - & - & 42,9 & 80,8 & 34,6 & C. + I.G. \\
\hline Cruzia sp. (L) & - & - & - & - & - & - & 14,3 & 11,0 & 1,6 & E. \\
\hline Mathevotaenia sp. & - & - & - & - & - & - & 14,3 & 6,5 & 0,9 & I.D. \\
\hline Physalopteridae (L) & 8,7 & 23,5 & 2,0 & - & - & - & 7,1 & 1,0 & 0,1 & $\mathrm{E}$. \\
\hline P. argentinensis & 26,1 & 8,0 & 1,4 & - & - & - & 50,0 & 5,0 & 2,5 & I.D \\
\hline Strongyloides sp. & 4,3 & 83,0 & 3,6 & - & - & - & - & - & - & I.D \\
\hline Trichohelix tuberculata & 95,7 & 102,7 & 102,7 & 100 & 116,08 & 116,08 & - & - & - & I.D. \\
\hline
\end{tabular}

E: Estómago, I.D: Intestino delgado, C: Ciego, I.G.: Intestino grueso

\subsubsection{Estructura de la comunidad parasitaria.}

Las distribuciones de las frecuencias de la prevalencia de parásitos para ambos hospedadores resulto ser bimodal (Figura 5.3). El coeficiente de correlación entre prevalencia e intensidad media fue no significativo para $C$. villosus $(r=0,52 ; p>0,05)$ y positivo y significativo para Z. pichiy $(r=0,73 ; p<0,05)$. Por lo tanto el análisis de especies centrales y satélites solo se realizó para Z. pichiy.
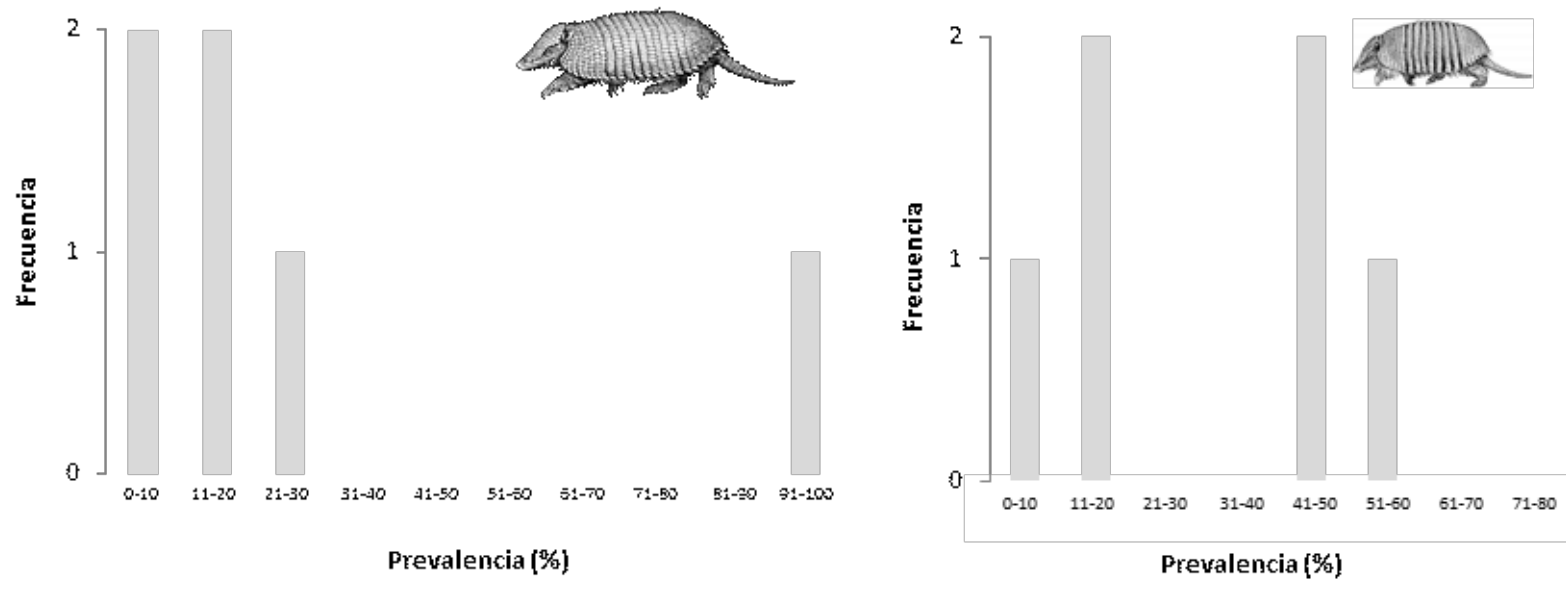

Figura 5.3 Distribución de frecuencias de las prevalencias de parásitos en las poblaciones de Patagonia sur de $C$. villosus (derecha) y Z. pichiy (izquierda). Ilustraciones modificadas de Díaz y Barquez (2002)y Parera y Erize (2002) 
En las Figuras 5.4 se muestra la estructura de la comunidad de helmintos de $Z$. pichiy para Patagonia sur. Se pudieron identificar dos especies centrales, Aspidodera fasciata y A. scoleciformis. Pterigodermatites argentinenis resulta ser un caso confuso, si bien la distribución de las frecuencias de prevalencias no mostró una disposición trimodal dada la baja intensidad media y alta prevalencia este helminto no podría clasificarse como especie satélite ni como especie central.

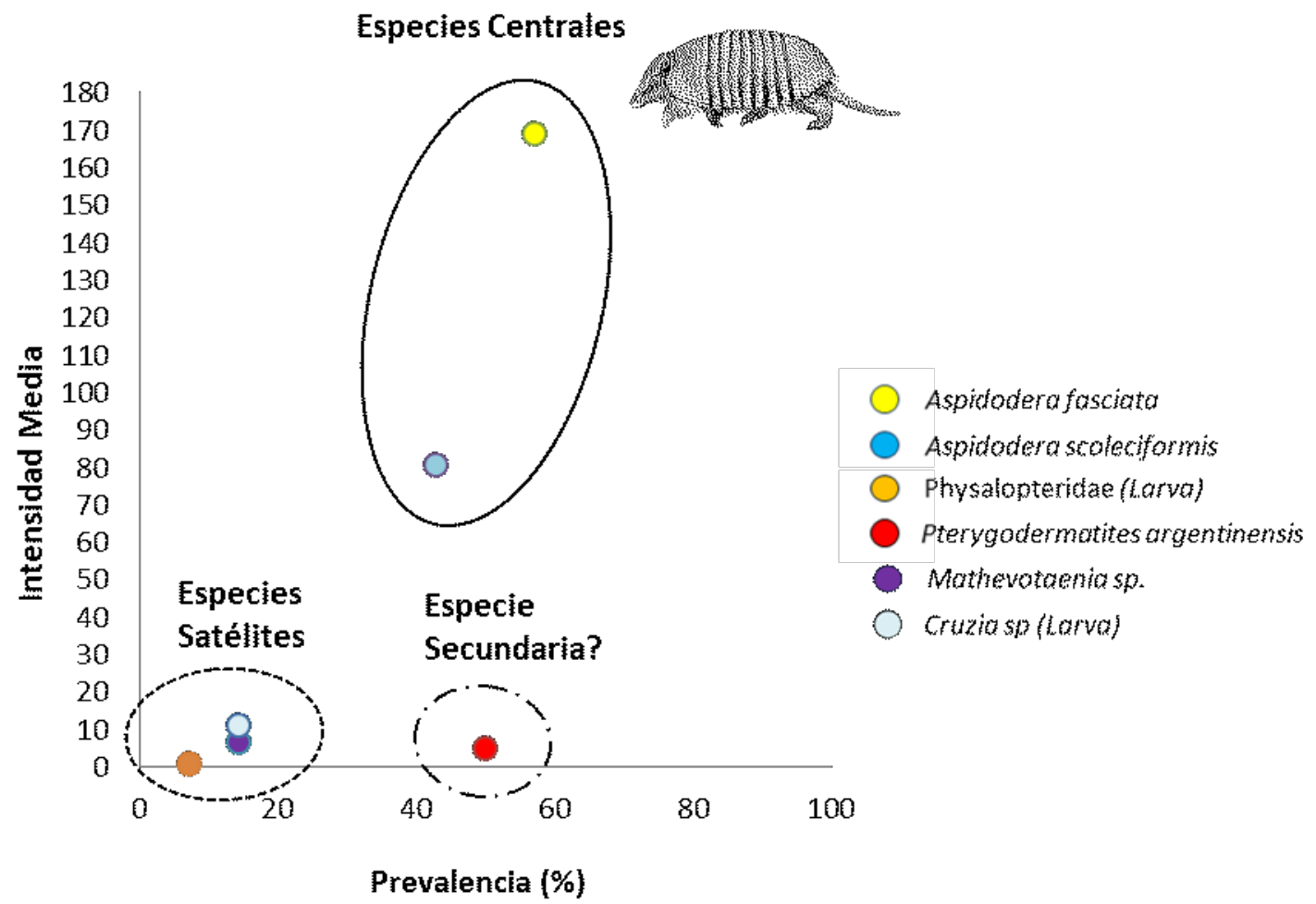

Figura 5.4 Estructura de la comunidad de helmintos de Z. pichiy. Ilustración modificada de Díaz y Barquez (2002) y Parera y Erize (2002)

En la Tabla 5.2 se observa que la población de Patagonia sur de $C$. villosus presenta tres especies parásitas co-dominantes: T. tuberculata, Aspidodera fasciata y $A$. scoleciformis, dos especies subordinadas: Pterygodermatites argentinensis y Strongyloides sp. (Grassi, 1879) (Rhabditida: Strongyloididae), mientras que la población fueguina presente una única especie dominante (T. tuberculata). Para Z. pichiy se observaron 
también tres especies co-dominantes ( $A$. fasciata, $A$. scoleciformis y $P$ argentinensis). Para ambos hospedasdores, las larvas de Physalopteridae y Cruzia sp. (Travassos, 1917) (Nematoda: Cruzidae) fueron clasificadas como pioneros no satisfactorios.

Tabla 5.2. Índice de importancia (I) para cada especie parásita encontrada en las tres poblaciones de $C$. villosus y $Z$. pichiy de Patagonia analizadas en esta tesis.

\begin{tabular}{|c|c|c|c|}
\hline \multirow[t]{2}{*}{ Parásitos } & \multicolumn{2}{|c|}{ Chaetophractus villosus } & \multirow{2}{*}{$\begin{array}{l}\text { Zaedyus pichiy } \\
\text { Patagonia sur }\end{array}$} \\
\hline & Patagonia sur & Tdf & \\
\hline Aspidodera fasciata & 0,16 & - & 0,74 \\
\hline Aspidodera scoleciformis & 0,02 & - & 0,22 \\
\hline Cruzia sp. (L) & - & - & 0 \\
\hline Mathevotaenia sp. & - & - & 0,002 \\
\hline Physalopteridae (L) & 0 & - & 0 \\
\hline P. argentinensis & 0,004 & - & 0,01 \\
\hline Strongyloides sp. & 0,001 & - & - \\
\hline Trichohelix tuberculata & 0,79 & 1,0 & - \\
\hline
\end{tabular}

\subsubsection{Zaedyus pichiy: Población Norte vs. Población Patagonia Sur}

Para la población Norte de Z. Pichiy, Ezquiaga et al. (2009) y Ezquiaga (2013) describieron una riqueza y diversidad de helmintos mayor $\left(S=11 ; H^{\prime}=1,08\right)$ que para la población de Patagonia Sur $\left(S=6 ; H^{\prime}=0,75\right)$ analizada en esta Tesis. Solo se comparten 4 especies entre ambas poblaciones de hospedadores (Tabla 5.3). Se encontraron diferencias significativas entre las prevalencias de ambas poblaciones solo para las especies del género Aspidodera, $A$. fasciata $\left(X^{2}=16,1 ; p<0,001 ; g l=1\right)$ y $A$. scoleciformis $\left(X^{2}=7.8 ; p<0,005\right)$. 
Tabla 5.3. Prevalencia de las especies de la comunidad componente de la población de Patagonia Norte y Sur de Zaedyus pichiy. $\mathrm{H}^{\prime}=$ índice de diversidad de Shannon-wiener.

\begin{tabular}{lcc}
\hline Parásito & $\begin{array}{c}\text { Población Norte } \\
\text { (Ezquiaga, 2013) } \\
\mathrm{H}^{\prime}=1,08\end{array}$ & $\begin{array}{c}\text { Población } \\
\text { Patagonia Sur } \\
\text { (Esta Tesis) } \\
\mathrm{H}^{\prime}=0,75\end{array}$ \\
\hline Ancylostoma caninum & 18 & - \\
Aspidodera fasciata & 100 & 50 \\
Aspidodera scoleciformis & 73 & 42,9 \\
Cyclobulura superinae & 27 & - \\
Cruzia sp. (L) & - & 14,3 \\
Delicata ransomi & 23 & - \\
Mathevotaenia sp. & 23 & 14,3 \\
Orihelia anticlava & 4 & - \\
Pterygodermatites cheatophracti & 23 & - \\
Pterygodermatites argentinensis & - & 50 \\
Physalopteridae (L) & - & 8,7 \\
Strongyloides sp. & 9 & - \\
Travassosia sp. & 5 & - \\
Trichohelix tuberculata & 23 & - \\
\hline
\end{tabular}

\subsubsection{Chaetophractus villosus: Población Núcleo vs. Población Patagonia Sur}

En la población núcleo (Ezquiaga, 2013), la riqueza específica y la diversidad de helmintos es mayor $\left(S=11 ; H^{\prime}=1,10\right)$ que en la población Patagonia Sur $\left(S=6 ; H^{\prime}=0,95\right)$. Solo 4 especies de helmintos son compartidas entre ambas poblaciones (Tabla 5.4). Se encontraron diferencias significativas para Aspidodera fasciata $\left(X^{2}=2,39 ; p<0.001 ; g l=1\right)$; A. scoleciformis $\left(X^{2}=5,44 ; p<0.001 ; g l=1\right)$ y Strongyloides sp $\left(X^{2}=1,19 ; p<0.001 ; g l=1\right)$. Por otra parte, Trichohelix tuberculata mantiene una prevalencia similar en ambas poblaciones (tabla 5.4). 
Tabla 5.4. Prevalencias de las especies de la comunidad componente para las poblaciones Núcleo y Patagonia Sur de Chaetophractus villosus.

\begin{tabular}{lcc}
\hline Parásito & $\begin{array}{c}\text { Población Núcleo } \\
\text { (Ezquiaga, 2013) } \\
\mathrm{H}^{\prime}=1,10\end{array}$ & $\begin{array}{c}\text { Población } \\
\text { Patagonia Sur } \\
\text { (Esta Tesis) } \\
\mathrm{H}^{\prime}=0,95\end{array}$ \\
\hline Ancylostoma caninum & 56 & - \\
Aspidodera fasciata & 96 & 39,1 \\
Aspidodera scoleciformis & 96 & 17,4 \\
Delicata ransomi & 26 & - \\
Mazzia bialata & 22 & - \\
Moennigia sp. & 9 & - \\
Mathevotaenia sp. & 26 & - \\
Orihelia anticlava & 17 & - \\
Pterygodermatites cheatophracti & 9 & - \\
Pterygodermatites argentinensis & - & 26,1 \\
Strongyloides sp. & 22 & 4,3 \\
Trichohelix tuberculata & 96 & 95,7 \\
\hline
\end{tabular}

\subsubsection{Chaetophractus villosus: Población TdF 2002-2006 vs. Población TdF Actual}

Ezquiaga et al. (2016) analizaron la diversidad de helmintos de la población de peludos introducidas en Tierra del Fuego, con individuos recolectados entre 2002 y 2006. Se encontró una única especie de nematode, Trichohelix tuberculata, con una prevalencia de $89 \%$. En esta Tesis se encontró que $T$. tuberculata sigue siendo la única especie de helminto para $C$. villosus de Tierra del Fuego. La prevalencia encontrada en esta Tesis fue de $100 \%$ y no se encontraron diferencias significativas $\left(X_{2}=0,640 ; p=0,423 ; \mathrm{gl}=1\right)$ respecto a lo estudiado por Ezquiaga et al. (2016).

\subsection{DISCUSIÓN Y CONCLUSIONES}

Se analizaron las comunidades de parásitos para poblaciones patagónicas del piche y el peludo. Para esta última especie, además, se realizó el mismo análisis para ejemplares de la población introducida en Tierra del Fuego para comparar con un análisis anterior 
(Ezquiaga et al., 2016). Con este estudio se completa la descripción general de la comunidad de parásitos de Z. pichiy y de C. villosus a lo largo de la región patagónica.

\subsubsection{Variaciones en la helmintofauna entre poblaciones Norte y Patagonia Sur de} Zaedyus pichiy

La disminución de la riqueza específica y de la diversidad de parásitos para la población de Zaedyus pichiy de Patagonia es aproximadamente la mitad con respecto al centro del país (Ezquiaga, 2013). Se hallaron solo tres especies parásitas coincidentes con las descritas por Ezquiaga et al. (2009) y Ezquiaga (2013) en la distribución Norte de este armadillo. De éstas, dos son nematodes, Aspidodera fasciata y A. scoleciformis y un cestode, Mathevotaenia sp. (Akumyan, 1946) (Cestoda: Anoplocephalidae).

La presencia de larvas de Physalopteridae es ocasional ya que se trata de un helminto cuyos hospedadores definitivos son preferentemente carnívoros, marsupiales y aves rapaces, mientras que sus hospedadores intermediarios son artrópodos (Rios, 2020). Algo similar ocurre con las larvas de Cruzia sp. en su distribución Patagónica. Este género de helmintos es parásito de marsupiales y es poco común en dasipódidos, encontrándose solo de manera ocasional (Adnet et al., 2009).

Por otro lado la presencia de Pterygodermatites argentinensis este trabajo, podría suponer un nuevo registro para algunas localidades de Patagonia. Sin embargo, $P$. argentinensis ya fue descrito para Z. pichiy en Chubut y Río Negro por Ezquiaga et al (2017).

La similitud entre las comunidades parásitas de dos localidades decrece a medida que aumenta la distancia entre dichas localidades (Soininen et al., 2007; Poulin y Krasnov, 2010). Y a su vez la latitud parece ser un factor muy importante en cuanto a la riqueza de parásitos de una población hospedadora (Nunn et al., 2005; Preisser, 2019). La disminución de especies de helmintos y la menor prevalencia prevalencia en la población de Patagonia Sur de Z. pichiy respecto a la población Norte (Ezquiaga et al., 2009 y Ezquiaga, 2013) puede deberse a múltiples factores, entre los cuales podrían nombrarse la baja tolerancia de determinados parásitos a las características climáticas que presenta la Patagonia centro-sur (sobre todo estadios larvales) y la disminución de la riqueza y la 
diversidad de artrópodos que operan como hospedadores intermediarios para muchos parásitos (Nunn et al., 2005; Poulin and Leung, 2011; Preisser, 2019).

\subsubsection{Variaciones en la helmintofauna de las poblaciones Núcleo y Patagónicas}

\section{de Chaetophractus villosus}

Al igual que para Zaedyus pichiy, la disminución de la riqueza específica y de la diversidad de helmintos de Patagonia es aproximadamente la mitad con respecto a la región núcleo (Ezquiaga, 2013). Entre las especies compartidas, ya descritas por Ezquiaga (2013), se encuentran Trichohelix tuberculata, Aspidodera fasciata, A. scoleciformis y Strongyloides sp. Además $T$. tuberculata es la única que se mantiene con una prevalencia alta $(97,1 \%)$ similar a la de la población núcleo $(96 \%)$ y es la única presente en Tierra del Fuego desde el primer análisis realizado allí (Ezquiaga et al., 2016). Si bien la presencia de Pterygodermatites argentinensis no implica un nuevo registro para Patagonia, se trataría de una nueva asociación parásito-hospedador. A su vez la presencia de larvas de Physolapteridae se trata de un registro ocasional ya que son parásitos de marsupiales (Adnet et al., 2009).

A diferencia de Zaedyus pichiy, la presencia del peludo en Patagonia es relativamente reciente. Por diversas causas, sobre todo antrópicas, logró dispersar por Patagonia hace aproximadamente 100 años (Abba et al., 2014). Las especies de parásitos que están ausentes en Patagonia corresponden a aquellas que en la región núcleo poseen una prevalencia menor al 50\%. Al tratarse de una dispersión "facilitada" por el ho mbre, es posible que aquellos hospedadores que se movilizaron hacia Patagonia representaran solo una porción pequeña de la población núcleo y quizás muchos de ellos no se encontraban parasitados con esos helmintos o simplemente esas especies de helmintos no pudieron sobrevivir a las condiciones climáticas Patagónicas o a la falta de hospedadores intermediarios, tal como es común a medida que aumenta la latitud (Nunn et al., 2005; Poulin and Leung, 2011; Preisser, 2019). Algunos de los parásitos que pierde la población Patagónica de $C$. villosus poseen un ciclo de vida indirecto, es decir, que requieren de un hospedador intermediario antes de ingresar al hospedador definitivo. Tal es el caso del 
cestode Mathevotaenia sp. Este helminto es muy común en xenartros y utiliza como hospedadores intermediarios a larvas de coleópteros y lepidópteros (Bienek and Klikoff, 1974). Éstos ítems presa son bastante común en la dieta de armadillos de Patagonia (Ver Capítulo 4; Gallo et al., 2019). Además este cestode también está presente en Z. pichiy de Patagonia (Tabla 5.1) al igual que el nematode $P$. argentinensis que también utiliza insectos como hospedadores intermediarios y sí está presente en ambos armadillos. En este contexto, no sería posible atribuir la pérdida de Mathevotaenia sp. a la falta de hospedadores intermediarios o a las condiciones climáticas de la Patagonia, por lo que esta variación podría atribuirse a la reciente dispersión del peludo en Patagonia. La pérdida de otros helmintos de ciclo de vida indirecto (Cyclobulura superinae, Orihelia anticlava, Travassosia sp.) sí podría deberse a la falta de hospedadores intermediarios en Patagonia. Por otro lado, los parásitos presentes en Patagonia son generalistas, esto les permite parasitar más de una especie hospedadora y así tener más posibilidades de expandir su distribución.

Respecto a la población invasora de C. villosus en Tierra del Fuego, Torchin et al. (2003) considera que solo aquellos parásitos con las prevalencias más altas acompañan a sus hospedadores en la nueva región invadida. Tal es el caso de T. tuberculata. Este helminto sigue siendo la única especie presente en la Isla Grande de Tierra del Fuego al igual que lo analizado por Ezquiaga et al. (2016). Al analizar este caso de co-introducción parásito-hospedador, el único cambio que se observa es un aumento en la prevalencia de T. tuberculata para la población introducida de $C$. villosus. El peludo fue introducido en la Isla Grande a principios de la década de 1980 (Poljak et al., 2007). Logró establecerse e invadir el ambiente fueguino de manera exitosa (Ver Capítulo 2). Veinte años más tarde (2002-2006) se observa que C. villosus había perdido prácticamente todas sus especies parásitas y no había incorporado ningún otro helminto presente en mamíferos de la Isla (Ezquiaga et al., 2016), a diferencia de lo que suele ocurrir en las especies hospedadoras introducidas (Torchin et al., 2003). Los resultados de esta tesis (12 años después) indican que la helmintofauna del peludo en Tierra del Fuego se mantiene sin cambios, solo con un leve aumento de la prevalencia de $T$. tuberculata. Esta especie de helminto es un 
nematode muy común en los armadillos de la tribu Euphractini (ver Capítulo 1) y se caracteriza por tener un estadio larval infectivo (L3) de vida libre que mantiene la cutícula del estadio larval previo (L2), lo que le permite soportar condiciones climáticas adversas (Anderson, 2000). Como plantearon Ezquiaga et al. (2016), es posible que la alta prevalencia de este nematode en toda la región Patagónica (Continental y Tierra del Fuego) sea por esta razón.

\subsubsection{Estructura de la comunidad de helmintos de Zaedyus pichiy y Chaetophractus villosus}

A pesar de la disminución de especies de helmintos, la población de Patagonia Sur de Z. pichiy (Figura 5.4) presenta una estructura comunitaria similar a la población Norte analizada por Ezquiaga (2013). Se mantienen las dos especies de Aspidodera como especies centrales y según el índice de Importancia, ambos helmintos son clasificados como parásitos "co-domiantes", contribuyen de manera significativa a la comunidad de parásitos de este armadillo. No obstante, la disminución en la prevalencia puede deberse a que tanto las interacciones entre hospedadores como la diversidad de especies hospedadoras que comparten esa especie de helminto es menor en Patagonia Sur que en regiones más al norte del país. Si bien $P$. argentinensis resultó ser un caso confuso para el análisis de especies satélites y centrales, el Índice de Importancia permitió categorizarlo como especie co-dominante, al igual que ambas especies de Aspidodera y, lo que significa que estas tres especies parásitas contribuyen de manera importante a la comunidad de helmintos de Z. pichiy en Patagonia sur.

Para el caso de Chaetophractus villosus el Índice de Importancia permitió categorizar a T. tuberculata, A. fasciata y A. scoleciformis como especies co-dominantes, destacando la importante contribución de las tres especies en la comunidad de helmintos de $C$. villosus de Patagonia sur. Aunque en esta tesis la estructura de la comunidad de helmintos para el peludo en Patagonia no pudo ser analizada según las especies centrales y satélites, estos resultados podrían coincidir con la estructura comunitaria propuesta por Ezquiaga (2013), donde se categoriza a Trichohelix tuberculata, Aspidodera fasciata y A. 
scoleciformis como especies centrales. No obstante, la gran disminución de la prevalencia de las dos especies de Aspidodera y la perdida de otros helmintos presentes con distribución Patagónica y presentes en la población de Patagonia sur de Z. pichiy (e.g. Matevothaenia sp; Tabla 5.3) podría deberse a la reciente dispersión del peludo en Patagonia. Numerosos estudios de co-invasión parásito-hospedador (Torchin et al., 2003; Lafferty et al., 2010) aseguran que la prevalencia y la riqueza específica de parásitos de hospedadores introducidos disminuye respecto a las poblaciones nativas. Sin embargo, a pesar de la disminución en la prevalencia en las dos especies de Aspidodera de Patagonia sur, la intensidad de infección y su contribución a la comunidad es alta, por lo que sería posible que los ejemplares de $C$. villous que dispersaron hacia Patagonia sur fueran portadores de un subset de dichas especies parásitas. Y a su vez, al tratarse de parásitos generalistas presentes también en Z. pichiy lograron dispersar junto con el hospedadores y mantenerse relativamente estables dentro de la comunidad de helmintos del peludo en Patagonia sur.

\subsubsection{Nuevos registros de helmintos en Patagonia Sur}

En esta tesis se encontraron nuevos registros de parásitos para algunas localidades de Patagonia, lo que permite ampliar sus rangos de distribución geográfica. Aspidodera fasciata se distribuye desde la provincia del Chubut hasta el sur de E.E.U.U (Jiménez Ruiz y Gardner, 2003; Ezquiaga, 2013). Aspidodera scoleciformis según Jiménez Ruiz y Gardner (2003) abarca desde el centro de Argentina hasta el sur de los E.E.U.U. Pterygodermatites argentinensis, descrito por Ezquiaga et al. (2017), presenta una distribución registrada hasta la provincia del Chubut. Para Strongyloides sp. la localidad más austral registrada es para el sur de la provincia de Buenos Aires (Ezquiaga, 2013). Lo mismo ocurre para el género del cestode Mathevotaenia sp. cuyo límite austral es la provincia de Río Negro (Ezquiaga, 2013). En este Capítulo, las especies antes detalladas amplían su límite austral de distribución geográfica hasta la provincia de Santa Cruz, Argentina, es decir, toda Patagonia excepto Tierra del Fuego.

En este contexto, la población invasora de $C$. villosus en Tierra del Fuego es la que continúa presentando mayor variación en la comunidad de parásitos respecto a la 
población nativa. Esto se debe a su reciente introducción (30 años aproximadamente). Lo que pueda ocurrir en los próximos años respecto a la parasitofauna del peludo en Tierra del Fuego resulta incierto. Ya que es posible que la situación se mantenga igual o que en algún momento $C$. villosus adquiera algún nuevo parásito de otros mamíferos fueguinos, o inclusive se produzca una co-invasión parásito-hospedador, y T. tuberculata infecte a otras especies de la Isla Grande.

\section{BIBLIOGRAFÍA}

Abba, A. M., S. Poljak, M. Gabrielli, P. Teta, and U. F. J. Pardiñas. 2014. Armored invaders in patagonia: recent southward dispersion of armadillos (CINGULATA, DASYPODIDAE. Mastozoología Neotropical 21:311-318.

Abba, A. M., M. F. Tognelli, V. P. Seitz, J. B. Bender, and S. F. Vizcaíno. 2012. Distribution of extant xenarthrans (Mammalia: Xenarthra) in Argentina using species distribution models. Mammalia 76:123-136.

Adnet, F. A. O., D. H. S. Anjos, A. Menezes-Oliveira, and R. M. Lanfredi. 2009. Further description of Cruzia tentaculata (Rudolphi, 1819) Travassos, 1917 (Nematoda: Cruzidae) by light and scanning electron microscopy. Parasitology Research 104:1207-1211.

ANDERSON, R. C. 2000. Nematode parasites of vertebrates: their development and transmission. CABI publishing, New York.

BieneK, G. K., AND L. G. KLIKoff. 1974. Parasitological Evidence of Arthropods as Food for Dipodomys merriami vulcani. The American Midland Naturalist 91:251-253.

Bordes, F., S. Morand, B. R. Krasnov, AND R. Poulin. 2010. Parasite diversity and latitudinal gradients in terrestrial mammals. Pp. 89-98 in The Biogeography of Host-Parasite Interactions (S. Morand \& B. R. Krasnov, eds.). Oxford University Press, New York.

Bush, A., K. Lafferty, J. Lotz, And A. Shostak. 1997. Parasitology meets ecology on its own terms: Margolis et al revisited. Journal of Parasitology 83:575-583.

BUSH, A. O., AND J. C. Holmes. 1986. Intestinal helminths of lesser scaup ducks: patterns of association. Canadian Journal of Zoology 64:132-141. 
Cabrera, L. 1971. Fitogeografía de la República Argentina. Boletin de la Sociedad Argentina de Botánica 14:1-50.

Ezquiaga, C., M. C. Digiani, And G. T. Navone. 2012. A New Molineid (Nematoda: Trichostrongylina) Parasite of Dasypus hybridus (Xenarthra: Dasypodidae) from Argentina. Journal of Parasitology 98:1156-1160.

EzquiagA, M. C. 2013. Estudios parasitológicos En Dasypodidae (Mammalia , Xenarthra) de Argentina : El valor de la diversidad en la interpretación de las asociaciones parásito - hospedador - ambiente. Tesis doctoral. Facultad de Ciencias Naturales y Museo, Universidad Nacional de La Plata.

Ezquiaga, M. C., A. M. AbBa, And G. T. NAvone. 2016. Loss of helminth species diversity in the large hairy armadillo Chaetophractus villosus on the Tierra del Fuego Island, Argentina. Journal of Helmintology 90:145-248.

Ezquiaga, M. C., ANd G. T. Navone. 2014. A new species of Moennigia (Trichostrongylina: Molineidae) a Parasite of Chaetophractus Spp. (Xenarthra: Dasypodidae) from Argentina. Journal of Parasitology 100:500-503.

Ezquiaga, M. C., T. A. Rios, A. M. Abba, And G. T. Navone. 2017. A new Rictulariid (Nematoda: Spirurida) in Xenarthrans from Argentina and new morphological data of Pterygodermatites (Paucipectines) Chaetophracti. Journal of Parasitology 103:727735.

Ezquiaga, M. C., M. Superina, And G. T. Navone. 2009. Parásitos intestinales de Zaedyus pichiy (Xenarthra: Dasypodidae) en Mendoza, Argentina. Mastozoología Neotropical 16:309-319.

Gallo, J. A., L. Fasola, And A. M. AbBa. 2019. Armadillos as natural pests control? Food habits of five armadillo species in Argentina. Mastozoología Neotropical 26:117-127. Holmes, J. C. 1987. The structure of helminth communities. International Journal for Parasitology 17:203-208.

Jiménez Ruiz, F. A., AND S. L. GARDNer. 2003. Aspidoderid nematodes from Bolivian armadillos, with the description of a new species of Lauroia (Heterakoidea: Aspidoderidae). Journal of Parasitology 89:978-983. 
Khalil L. F., Jones A. ANd Bray R. A. 1994. Keys to the cestode parasites of vertebrates. CAB International, Wallingford, Reino Unido, $751 \mathrm{pp}$

Krasnov, B. R., G. I. Shenbrot, I. S. Khokhlova, and A. A. Degen. 2004. Flea species richness and parameters of host body, host geography and host "milieu." Journal of Animal Ecology 73:1121-1128.

Lafferty, K. D., M. E. Torchin, ANd A. M. KuRIS. 2010. Parasite, The geography of host and Invasions. Pp. 191-203 in The Biogeography of Host-Parasite Interactions (B. R. Krasnov \& S. Morand, eds.). Oxford University Press, New York.

Lindenfors, P., C. L. Nunn, K. E. Jones, A. A. Cunningham, W. Sechrest, and J. L. Gittleman. 2007. Parasite species richness in carnivores: Effects of host body mass, latitude, geographical range and population density. Global Ecology and Biogeography 16:496-509.

LUQUe, J. L., AND R. Poulin. 2008. Linking ecology with parasite diversity in Neotropical fishes. Journal of Fish Biology 72:189-204.

Lymbery, A. J., M. Morine, H. G. Kanani, S. J. Beatty, And D. L. Morgan. 2014. Co-invaders: The effects of alien parasites on native hosts. International Journal for Parasitology: Parasites and Wildlife 3:171-177.

Matteuccl, S. D. 2011. Ecorregión Estepa Patagónica. Pp. 549-654 in Ecorregiones y complejos ecosistémicos argentinos (J. Morello, S. D. Matteucci, A. F. Rodríguez \& M. Silva, eds.)., Orientación Grafica S.R.L., Buenos Aires.

Maurer, B. A., AND B. J. McGill. 2011. Measurement of species diversity. Pp. 55-65 in Biological Diversity: Frontiers in measurement and assessment (B. J. Magurran, A. E. \& McGill, ed.). Oxford University Press.

Morales G. AND L. A. PINO. 1987. Parasitología cuantitativa. Fundación Fondo Editorial. Acta Científica Venezolana Eds. 332pp.

NAVONE, G. T. 1990. Estudio de la distribución, porcentaje y microecología de los parásitos de algunas especies de edentados argentinos. Studies on Neotropical Fauna and Environment 25:199-210. 
Nunn, C. L., S. M. Altizer, W. Sechrest, And A. A. Cunningham. 2005. Latitudinal gradients of parasite species richness in primates. Diversity and Distributions 11:249-256.

Poljak, S., V. Confalonieri, M. Fasanella, M. Gabrielli, and M. Lizarralde. 2010. Phylogeography of the armadillo Chaetophractus villosus (Dasypodidae Xenarthra): Post-glacial range expansion from Pampas to Patagonia (Argentina). Molecular Phylogenetics and Evolution 55:38-46.

Poljak, S., J. Escobar, G. Deferrari, and M. Lizarralde. 2007. A new introduced mammal in Tierra del Fuego: the "large hairy armadillo" Chaetophractus villosus (Mammalia, Dasypodidae) in the Isla Grande island. Revista Chilena de Historia Natural 80:285294.

Poulin, R. 1995. Phylogeny, ecology, and the richness of parasite communities in vertebrates. Ecological Monographs 65:283-302.

POULIN, R., AND B. R. KRASNOV. 2010. Similarity and variability of parasite assemblages across geographical space. Pp. 115-127 in The Biogeography of Host-Parasite Interactions (S. Morand \& B. R. Krasnov, eds.). Oxford University Press, New York.

Poulin, R., AND T. L. F. LEUNG. 2011. Latitudinal gradient in the taxonomic composition of parasite communities. Journal of Helminthology 85:228-233.

PREISSER, W. 2019. Latitudinal gradients of parasite richness: a review and new insights from helminths of cricetid rodents. Ecography 42:1315-1330.

RIBICICH, M. ET AL. 2010. Trichinella infection in wild animals from endemic regions of Argentina. Parasitology Research 107:377-380.

RıOS, T. A. 2020. Estudios parasitológicos en xenartros (MAMMALIA: XENARTHRA) del Chaco argentino. Tesis doctoral. Facultad de Ciencias Naturales y Museo, Universidad Nacional del La Plata.

Sikes, R. S., ANd A. C. AND U. C. of the A. S. of Mammalogists. 2016. 2016 Guidelines of the American Society of Mammalogists for the use of wild mammals in research and education. Journal of mammalogy 97:663-688.

Soininen, J., R. McDonald, ANd H. Hillebrand. 2007. The distance decay of similarity in ecological communities. Ecography 30:3-12. 
SUPERINA, M. ET AL. 2019. Zaedyus pichiy. En: SAyDS-SAREM (eds.) Categorización 2019 de los mamíferos de Argentina según su riesgo de extinción. Lista Roja de los mamíferos de Argentina. Versión digital: http://cma.sarem.org.ar

Thul J., D. Forrester ANd C. AbercombiRe. 1985. Ecology of parasitic helminths of wood ducks, Aix sponsa, in the Atlantic Flyway. Proceedings of the Helminthological Society of Washington 52:297-310.

Torchin, M. E., K. D. Lafferty, A. P. Dobson, V. J. Mckenzie, and A. M. Kuris. 2003. Introduced species and their missing parasites 421:628-630. 


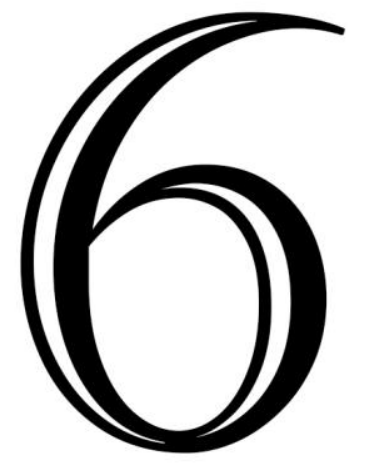

\section{CONCLUSIONES FINALES Y RECOMENDACIÓNES DE MANEJO}

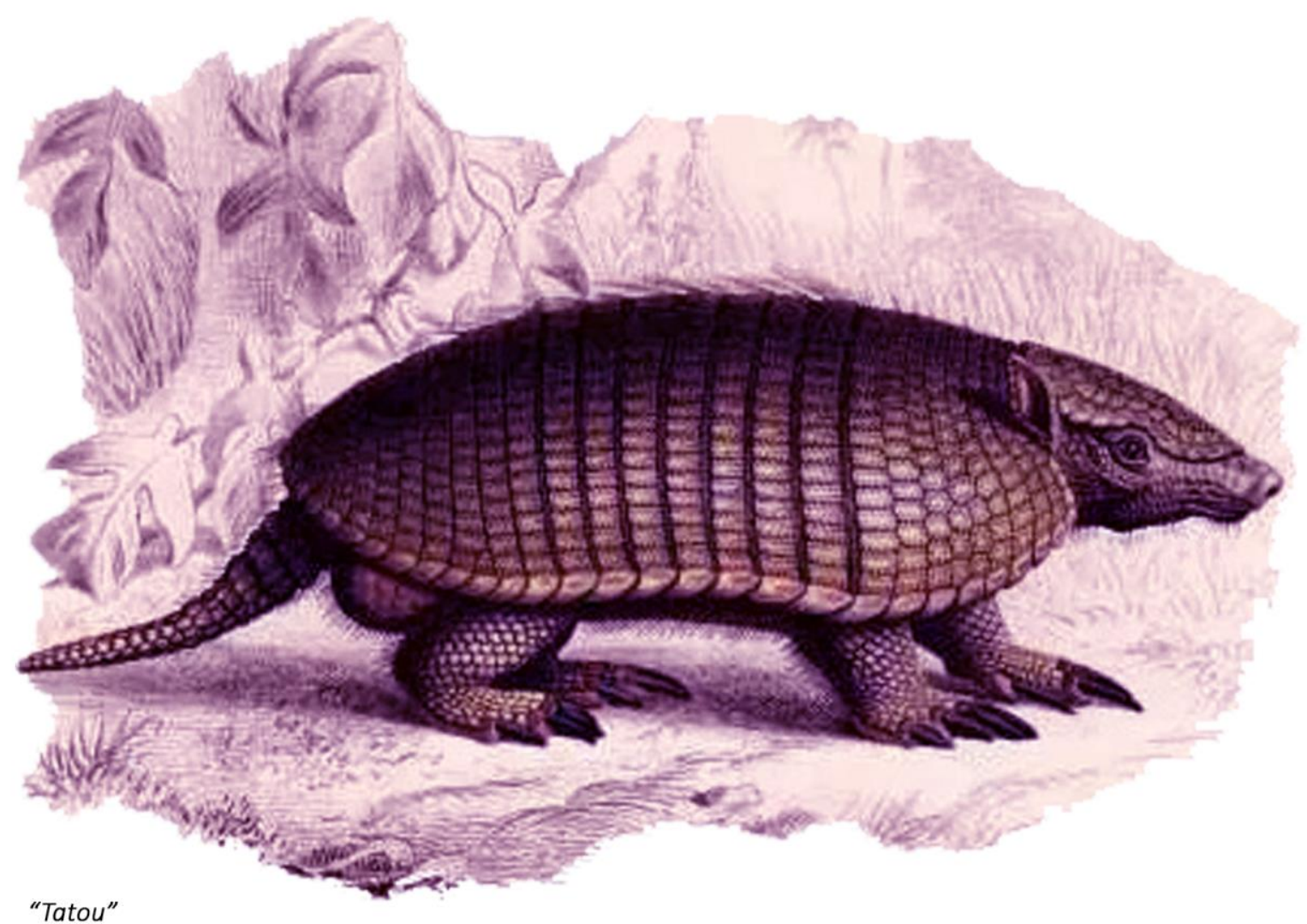

Alcide D'Orbigny. Dictionnaire universel d'histoire naturelle: atlas. Vol I. Paris, L. Houssiaux et cie. 1861

"Arriba de aquel quebracho estaba un peludo colí, se cayó' el quebracho y allá va el añamebui" 


\subsection{La invasión exitosa del peludo en Tierra del Fuego}

En esta tesis se obtuvo información actualizada acerca de la distribución, dieta, parasitología e impacto sobre especies nativas de Chaetophractus villosus en la Isla Grande de Tierra del Fuego.

4. La distribución de la población invasora de C. villosus en Tierra del Fuego se incrementó notablemente desde el último estudio publicado por Poljak et al. (2007), ocupando toda la porción norte de la Isla Grande.

... La mayor densidad de evidencias indirectas de peludos se encontró en zonas elevadas no inundables, con una altura de la vegetación media-alta y con suelos de tipo húmico.

A. No se observó asociación del peludo con ninguna característica del paisaje, incluyendo la red de gasoductos.

2.. La principal vía de introducción de este armadillo fue a través de la translocación de individuos desde el continente por parte de transportistas de las industrias.

La translocación de individuos podría haber sido uno de los mecanismos por los cuales el peludo logró superar la barrera que suponen los ríos Chico y Grande.

A.. La tasa de expansión del peludo en la Isla Grande fue de 10,9km/año.

A. Se distinguieron dos zonas de mayor actividad de peludos. Una zona A, coincidente con el sitio de introducción en la región de San Sebastián, y una Zona B, al sur de Río Grande, considerada como el frente sur de la invasión.

A. El peludo es considerado dañino por parte de la población rural debido a que la construcción de cuevas podría generar lesiones en las extremidades del ganado.

En principal uso del peludo por parte de la población local es la caza para consumo.

2. La diversidad de la dieta del peludo en Tierra del Fuego es menor que en su distribución nativa y la evaluación de depredación sobre aves que nidifican en el suelo no permite concluir que este armadillo deprede activamente sobre huevos de aves en la Isla. 
Las variaciones en la diversidad de helmintos de ambos armadillos en Patagonia centro-sur respecto a poblaciones al norte del Río Chubut, podría deberse a la falta de hospedadores intermediarios o a la baja tolerancia de determinados parásitos a las características climáticas.

La gran disminución en la prevalencia de helmintos en la probación Patagónica de $C$. villosus respecto a la población núcleo, sumado a la perdida de parásitos que tienen distribución Patagónica, podría ser resultado de la reciente dispersión del peludo en Patagonia.

En Tierra del Fuego, la única especie parásita es Trichohelix tuberculata al igual que lo registrado en el último estudio realizado (Ezquiaga et al. 2016).

A. Se ampliaron los rangos de distribución geográfica de los nematodes Aspidodera fasciata, A. scoleciformis, Pterygodermatites argentinensis, Strongyloides sp. y del cestode Mathevotaenia sp. y se fija la provincia de Santa Cruz como el nuevo límite austral de sus distribuciones.

\subsection{Recomendaciones para el manejo del meludo en Tierra del}

\section{Fuego}

Al momento de estudiar una especie invasora, resulta importante distinguir la situación en la que se encuentra la población introducida en el proceso de invasión para poder tomar desiciones. La erradicación, una de las decisiones más comunes, debe solo si es altamente factible (Genovesi, 2005). De no ser posible la erradicación, se debe considerar generar planes de control a largo plazo (Sanguinetti et al., 2014).

En este contexto, los resultados obtenidos en esta tesis permitieron establecer que luego de su introducción (ver Poljak et al. 2007) C. villosus logró establecerse al ambiente fueguino. Logró dispersar hacia el sur y hacia el norte desde los puntos de introducción (Capítulo 2). La interpretación visual del mapa de calor (Figura 6.1) permite identificar y delimitar aquellas áreas de mayor concentración de actividad de peludos, que deben ser considerados al momento de implementar algún plan de manejo sobre este armadillo invasor. El área A corresponde al sitio de introducción y es el sitio de mayor densidad de 
cuevas y hozaduras. Allí, el peludo logró establecerse, seguramente favorecido por la presencia de gasoductos durante los primeros años y luego dispersar (Figura 6.1). El área B presenta menor densidad de signos de armadillos pero muy concentrados, lo que podría ser considerado como un frente de invasión. Seguramente el establecimiento en este área fue reciente, producto de la translocación de individuos (Figura 6.1) y dispersión natural facilitada por caminos y puentes que le permitieron al peludo atravesar los dos ríos de la zona.

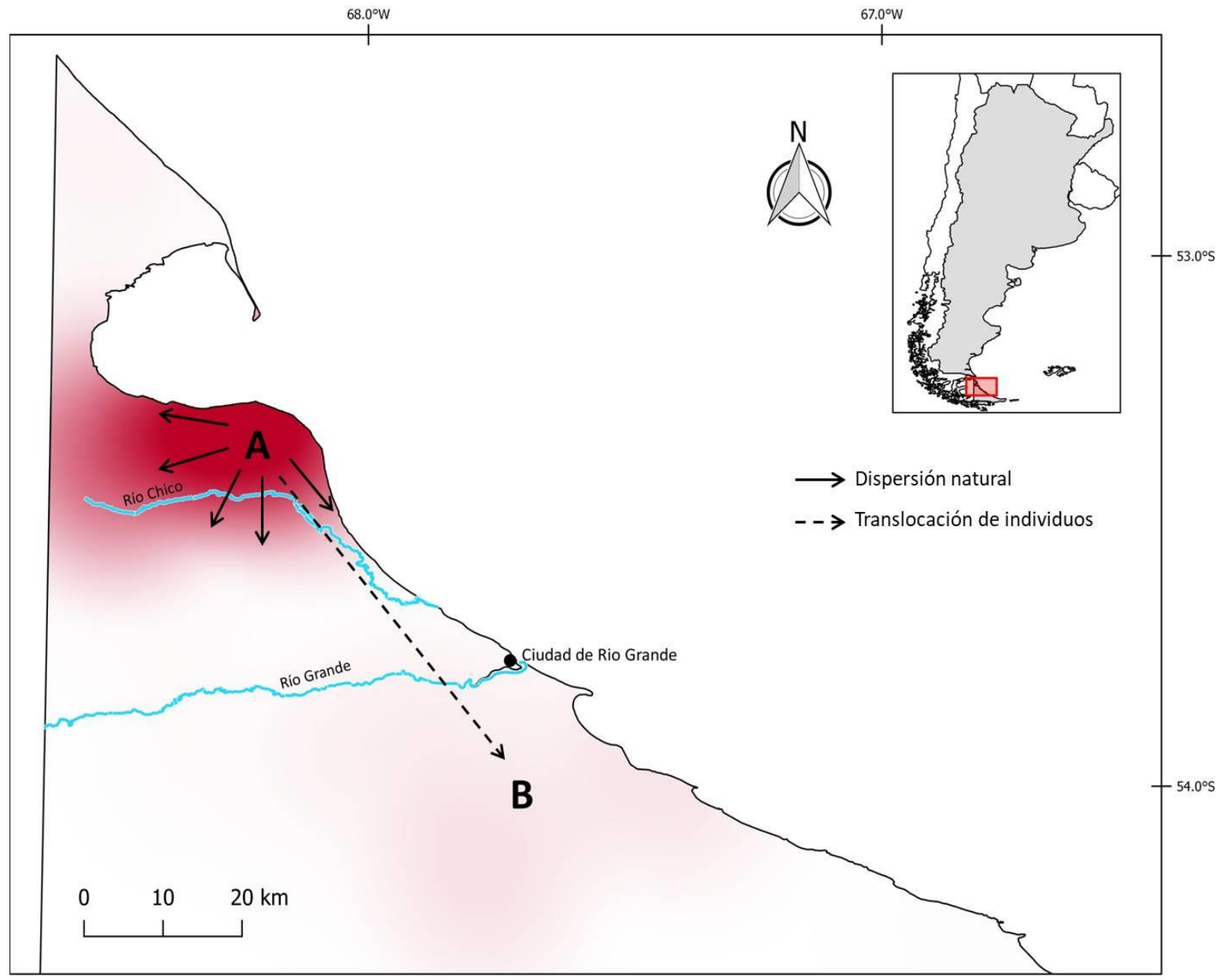

Figura 6.1. La intensidad del color rojo denota áreas de mayor densidad de evidencias de C. villosus en la Isla Grande de Tierra del Fuego. 
Ambas áreas deben ser consideradas al momento de realizar cualquier esfuerzo de manejo sobre $C$. villosus. Por un lado, el área A coincide con los sitios donde antiguamente los cauquenes (Chloephaga spp.) criaban con frecuencia, por lo que la presencia de peludos en la zona, podría agravar aún más la situación crítica de estas aves. Asimismo, en esa zona, se encuentra uno de los establecimientos agropecuarios con mayor concentración de ganado de la Isla Grande de

Tierra del Fuego, y por esto, el potencial conflicto ganadero-armadillo sería mayor que en otras partes de la Isla. Por otro lado, el área B corresponde al límite sur de la invasión, lo que implica que cualquier plan de control en esta área podría impedir el avance del peludo hacia el ecotono. Así mismo, es importante destacar que en la porción norte de la Isla Grande se distribuyen dos vertebrados endémicos de la zona. El área A coincide con la distribución de la lagartija magallánica, Liolaemus magellanicus (Avila et al., 2016), mientras que tanto el tarea A como el área B corresponden a la distribución de Ctenomys magellanicus (Sanchez y Lizarralde, 2019).

Teniendo en cuenta los resultados de esta tesis se proponen los siguientes lineamientos para ejecutar un plan de manejo del peludo en la Isla Grande de Tierra del Fuego:

$\rightarrow$ Considerar el área A como el sitio de mayor concentración de individuos.

$\rightarrow$ Considerar el área B como el frente de invasión sur a partir de donde el peludo puede invadir todo el ecotono de la Isla Grande.

$\rightarrow$ Tener en cuenta que, en dichas áreas, los peludos se concentraran en zonas con terrenos elevados no inundables (naturales o artificiales), con una altura de la vegetación medio/alta (coironales o matorrales de mata negra/verde) y con suelos húmicos (con alto contenido de materia orgánica).

$\rightarrow$ Llevar a cabo campañas de erradicación. Para este caso se recomienda la captura manual de individuos y el uso de trampas de captura vivía (tipo tomahawk) en las entradas de cuevas.

$\rightarrow$ Desarrollar campañas de difusión con el objetivo de concientizar a la población local acerca del problema que genera la translocación y cría de peludos. 
$\rightarrow$ Articular la campaña de difusión con un programa de detección temprana de nuevas áreas.

$\rightarrow$ Realizar modificaciones de estructuras y puentes que facilitan el cruce de ríos por parte del peludo hacia la zona $B$.

Considerando las dimensiones de la Isla Grande de Tierra del Fuego y la división política de la misma, es importante que cualquier plan de control se ejecute de manera binacional ya que la distribución del peludo abarca gran parte del territorio chileno de la Isla (Cabello et al., 2017; Poljak et al., 2020). En este contexto, esta invasión debe considerarse como un problema ecológico y social que necesita ser resuelto a través de medidas que impidan futuras colonizaciones de nuevas áreas de la Isla Grande de Tierra del Fuego.

\section{BIBLIOGRAFÍA}

Cabello, J. L., A. E. J. Valenzuela, And C. B. Anderson. 2017. Avance en el proceso de la invasión biológica del peludo Chaetophractus villosus (Dasypodidae) en la Isla Grande de Tierra del Fuego: un nuevo desafío binacional. Anales Instituto Patagonia (Chile) 45:109-115.

Ezquiaga, M. C., A. M. ABBA, AND G. T. NAvone. 2016. Loss of helminth species diversity in the large hairy armadillo Chaetophractus villosus on the Tierra del Fuego Island, Argentina. Journal of Helmintology 90:145-248.

GENOVESI, P. 2005. Eradications of invasive alien species in Europe: a review Piero. Biological Invasions:127-133.

MelLA-Romero, J., J. M. ÁvilA, F. R. CortéS, AND C. M. VilLoutA. 2020. Registro de nado y evidencias indirectas del peludo patagónico Chaetophractus villosus ( Desmarest , 1804 ) en Aysén , Chile 48:47-51.

Poljak, S., J. Escobar, G. Deferrari, And M. LizARRAlde. 2007. A new introduced mammal in Tierra del Fuego: the "large hairy armadillo" Chaetophractus villosus (Mammalia, Dasypodidae) in the Isla Grande island. Revista Chilena de Historia Natural 80:285-294.

Poljak, S., J. SÁnChez, L. LANUSSE, AND M. S. LizARRALDE. 2020. Anthropogenic invaders: Historical 
biogeography, current genetic status and distribution range of the "peludo" Chaetophractus villosus (Xenarthra) in Patagonia and Tierra del Fuego, southern South America. Mammalia.

SÁNCHEZ, J., AND M. S. LizARRALDE. 2019. Ctenomys magellanicus. Categorización 2019 de los mamíferos de Argentina según su riesgo de extinción. Lista Roja de los mamíferos de Argentina. (SAyDS-SAREM, ed.). SAyDS-SAREM.

SANGUINETtI, J. ET AL. 2014. Manejo de especies exóticas invasoras en Patagonia, Argentina: Priorización, logros y desafíos de integración entre ciencia y gestión identifcados desde la Administración de Parques Nacionales. Ecologia Austral 24:183-192. 


\section{BIBLIOGRAFÍA COMPLETA}

ABBA, A. M., y S. F. VizCaíno. 2011. Distribución de los armadillos (Xenarthra: Dasypodidae) en la provincia de Buenos Aires, Argentina. Mastozoología Neotropical 18:185-206.

ABBA, A. M., D. E. UdRIZAR, AND S. F. VizCAínO. 2005. Distribution and use of burrows and tunnels of Chaetophractus villosus ( Mammalia , Xenarthra ) in the Eastern Argentinean pampas. Acta theriologica 50:115-124.

Abba, A. M., E. Zufiaurre, M. Codesido, and A. D. N. Bilenca. 2016. Habitat use by armadillos in agroecosystems of central Argentina: does plot identity matter? Journal of Mammalogy 20:1-7.

AbBA, A. M., E. Zufiaurre, M. Codesido, ANd D. N. BilenCA. 2015. Burrowing activity by armadillos in agroecosystems of central Argentina: Biogeography, land use, and rainfall effects. Agriculture, Ecosystems and Environment 200:54-61.

AbBa, A. M., J. A. Gallo, And E. Zufiaurre. 2018. Uso de basurales por parte del peludo (Chaetophractus villosus). Notas sobre Mamíferos Sudamericanos 1:1-5.

AbBa, A. M., M. F. Tognelui, V. P. Seitz, J. B. Bender, And S. F. Vizcaíno. 2012. Distribution of extant xenarthrans (Mammalia: Xenarthra) in Argentina using species distribution models. Mammalia 76:123-136.

AbBa, A. M., M. J. NabTe, ANd D. E. U. SAUthier. 2010. New data on armadillos (Xenarthra: Dasypodidae) forc Patagonia, Argentina. Edentata 11:11-17.

Abba, A. M., S. Poljak, M. Gabrielli, P. Teta, And U. F. J. Pardiñas. 2014. Armored invaders in patagonia: recent southward dispersion of armadillos (CINGULATA, DASYPODIDAE. Mastozoologia neotropical 21:311-318.

ABBA, A.M. AND M.H. CASSINI. 2010. Ecological differences between two sympatric species of armadillos (Xenarthra, Mammalia) in a temperate region of Argentina. Acta Theriologica 55:35-44. 
Abba, A.M., M.F. Tognelli, V.P. Seitz, J.B. Bender and S.F. Vizcaíno. 2012. Distribution of extant xenarthrans (Mammalia: Xenarthra) in Argentina using species distribution models. Mammalia 76:123-136.

ABBA, A.M., S.F. VizCAÍnO AND M.H. CASSINI. 2007. Effects of land use on the distribution of three species of armadillos in the argentinean pampas. Journal of Mammalogy 88:502-507.

ABBA, A.M., S.F. VizCaíno y M.H. CASSINI. 2009. Eto-ecología y conservación de tres especies de armadillos (Dasypus hybridus, Chaetophractus villosus y C . vellerosus ) en el Noreste de la Provincia de Buenos Aires, Argentina. Edentata 8:41-47.

Adnet, F. A. O., D. H. S. Anjos, A. Menezes-Oliveira, And R. M. LAnfredi. 2009. Further description of Cruzia tentaculata (Rudolphi, 1819) Travassos, 1917 (Nematoda: Cruzidae) by light and scanning electron microscopy. Parasitology Research 104:1207-1211.

ANDERSON, R. C. 2000. Nematode parasites of vertebrates: their development and transmission. CABI publishing, New York.

ARbeiter, S., AND E. Franke. 2018. Predation risk of artificial ground nests in managed floodplain meadows. Acta Oecologica 86:17-22.

Arriagada, A., L. Baessolo, C. Saucedo, J.E. Crespo, J. Cerda, L. Parra, et Al. 2017. Hábitos alimenticios de poblaciones periféricas de Zaedyus pichiy y Chaetophractus villosus (Cingulata, Chlamyphoridae) en la Patagonia chilena. Iheringia. Série Zoologia 107:18.

Bienek, G. K., AND L. G. KLIKOfF. 1974. Parasitological Evidence of Arthropods as Food for Dipodomys merriami vulcani. The American Midland Naturalist 91:251-253.

BolLy P. 2002. Individual variation in metabolic traits of wild nine-banded armadillos (Dasypus novemcinctus), and the aerobic capacity model for the evolution of endothermy. The Journal of experimental biology 205:3207-3214. 
Bordes, F., S. Morand, B. R. KRASnov, ANd R. Poulin. 2010. Parasite diversity and latitudinal gradients in terrestrial mammals. Pp. 89-98 in The Biogeography of Host-Parasite Interactions (S. Morand \& B. R. Krasnov, eds.). Oxford University Press, New York.

Borromei A. M., J. F. Ponce, A. Coronato, M. S. Candel, D. Olivera and M. Okuda. 2014. Reconstrucción de la vegetación posglacial y su relación con el ascenso relativo del nivel del mar en el extremo este del canal beagle, Tierra del Fuego, Argentina. Andean Geology 41:362-379.

BUSH, A. O., AND J. C. HolmEs. 1986. Intestinal helminths of lesser scaup ducks: patterns of association. Canadian Journal of Zoology 64:132-141.

Bush, A., K. LAfFerty, J. LotZ, AND A. ShOSTAK. 1997. Parasitology meets ecology on its own terms: Margolis et al revisited. Journal of Parasitology 83:575-583.

Cabello, J.L., A.J. Valenzuela y C.B. Anderson. 2017. Avance en el proceso de la invasión biológica del peludo Chaetophractus villosus (Dasypodidae) en la Isla Grande de Tierra del Fuego: un nuevo desafío binacional. Anales Instituto Patagonia 45:109115.

CABRERA, L. 1971. Fitogeografía de la República Argentina. Boletín de la Sociedad Argentina der Botánica 14:1-50.

Camilo-Alves, C. D. S. E. P. And Mourão G.D.M. 2006. Responses of a specialized insectivorous mammal (Myrmecophaga tridactyla) to variation in ambient temperature. Biotropica 38:52-56.

CANEVARI, P. 1996. The austral geese (Chloephaga spp.) of Southern Argentina and Chile: a review of its current status. Gibier faune sauvage 13:355-366.

Ciuccio, M. 2014. Ecología comportamental de los dasipódidos en el pastizal pampeano, con particular consideración de los hábitos alimenticios. Enfoque eco-morfofisiológico. Universidad Nacional del Sur. 
Cossa, N.A., L. FASOlA, I. RoesLeR, AND J.C. RebOREDA. 2017. Ruddy-headed Goose Chloephaga rubidiceps: former plague and present protected species on the edge of extinction. Bird Conservation International 27:269-281.

D.G. DE E.Y.C. 2015. Boletín estadístico agropecuario. Ushuaia, Tierra del Fuego.

DICKMAN, C.R. 1996. Impact of exotic generalist predators on the native fauna of Australia. Wildlife Biology 2:185-195.

Ezquiaga, C., M. C. Digiani, And G. T. Navone. 2012. A New Molineid ( Nematoda : Trichostrongylina ) Parasite of Dasypus hybridus ( Xenarthra: Dasypodidae ) from Argentina A New Molineid ( Nematoda : Trichostrongylina ) Parasite of Dasypus hybridus. Journal of Parasitology 98:1156-1160.

Ezquiaga, M. C. 2013. Estudios Parasitológicos En Dasypodidae (Mammalia , Xenarthra) de Argentina: el valor de la diversidad en la interpretación de las asociaciones parásito hospedador - ambiente. Facultad de Ciencias Naturales y Museo, Universidad Nacional de La Plata.

EzQuiaga, M. C., A. M. AbBa, And G. T. Navone. 2016. Loss of helminth species diversity in the large hairy armadillo Chaetophractus villosus on the Tierra del Fuego Island, Argentina. Journal of Helmintology 90:145-248.

Ezquiaga, M. C., AND G. T. Navone. 2014. A New Species of Moennigia (Trichostrongylina: Molineidae) a Parasite of Chaetophractus Spp. (Xenarthra: Dasypodidae) from Argentina. Journal of Parasitology 100:500-503.

Ezquiaga, M. C., M. Superina, And G. T. NAvone. 2009. Parásitos intestinales de Zaedyus pichiy (Xenarthra: Dasypodidae) en Mendoza, Argentina. Mastozoología Neotropical 16:309-319.

Ezquiaga, M. C., T. A. Rıos, A. M. AbBa, And G. T. NAvone. 2017. A New Rictulariid (Nematoda: Spirurida) in Xenarthrans from Argentina and New Morphological Data of 
Pterygodermatites (Paucipectines ) chaetophracti. Journal of Parasitology 103:727735.

GallinA-Tessaro, S. 2011. Técnicas para conocer la dieta. P. 377 in Manual de técnicas para el estudio de la fauna. Volúmen I. (S. Gallina y C. López-González, eds.). Universidad Autónoma de Querétaro-Instituto de Ecología, Querétaro, México.

Gallo, J. A. et AL. 2019. Chaetophractus villosus. Categorización 2019 de los mamíferos de Argentina según su riesgo de extinción. Lista Roja de los mamíferos de Argentina. (SAyDS-SAREM, ed.).

Gallo, J. A., L. Fasola, AND A. M. AbBA. 2019. Armadillos as natural pests control? Food habits of five armadillo species in Argentina. Mastozoología Neotropical 26:117-127.

GeNOVESI, P. 2005. Eradications of invasive alien species in Europe: a review Piero. Biological Invasions: 127-133.

GoBSteR, P. H. 2011. Factors Affecting People's Responses to Invasive Species Management. Pp. 249-263 in Invasive and Introduced Plants and Animals: Human Perceptions, Attitudes and Approaches to Management Edited (I. D. Rotherham \& R. A. Lambert, eds.). EARTHSCAN, London - Washington, DC.

GrassÉ, P.P. 1955. Ordre des Edentés, Ordre des Pholidotes. Pp. 1182-1284, in: Traité de Zoologie: anatomie, systématique, biologie. Masson Press, Paris.

GROOMBRIDGE, B. 1992. Global biodiversity: status of the earth's living resources. Chapman \& Hall, London.

Grosman, F., G. GonzÁlez, P. Sanzano, y D. Agüería. 2002. Alimentación, nichos tróficos y competencia interespecífica de peces de la laguna de Monte , Argentina. Pp. 129140 in.

Grosman, F., P. Sanzano, D. Agüeria, G. GonzÁlez, y S. Sergueña. 2001. Ecología reproductiva, edad, crecimiento, condición y alimentación del pejerrey (Odontesthes bonariensis) 
en un ambiente del SO de la provincia de Buenos Aires, Argentina. Revista AquaTIC $12: 20$.

Grosman, M. F. 1995. Variaciones estacionales en la dieta del pejerrey (Odonthes bonariensis). Revista de la Asociación de Ciencias Naturales del Litoral 26:9-18.

Guichón, M. L., M. Monteverde, L. Piudo, J. Sanguinetti, And D. Martino. 2016. Mamíferos introducidos en la provincia de Neuquén: Estado actual y prioridades de manejo.

Guichón, M. L., V. V. Benitez, A. C. Gozzi, M. Hertzriken, And M. Borgnia. 2015. From a lag in vector activity to a constant increase of translocations: invasion of Callosciurus squirrels in Argentina. Biological Invasions 17:2597-2604.

HeRnández, C., S. SADE, y J. RAU. 2017. Dieta del jabalí (Sus scrofa), invasor biológico reciente del Parque Nacional Puyehue, sur de Chile. Mastozoología Neotropical 24:467-473.

HOLMES, J. C. 1987. The structure of helminth communities. International Journal for Parasitology 17:203-208.

HULME, P. E. 2009. Trade, transport and trouble: Managing invasive species pathways in an era of globalization. Journal of Applied Ecology 46:10-18.

JimÉnEz RuIZ, F. A., AND S. L. GaRdner. 2003. Aspidoderid nematodes from Bolivian armadillos, with the description of a new species of Lauroia (Heterakoidea: Aspidoderidae). Journal of Parasitology 89:978-983.

JOHNSON, D. H. 1980. The comparison of usage and availability measurements for evaluating resource. Ecology 61:65-71.

Khalil L. F., Jones A. ANd Bray R. A. 1994. Keys to the cestode parasites of vertebrates. CAB International, Wallingford, Reino Unido, $751 \mathrm{pp}$

KAMENOVA, S. ET AL. 2017. Invasions toolkit: current methods for tracking the spread and impact of invasive species. Advances in Ecological Research. 1st edition. Elsevier Ltd. 
KAPITZA, K., H. Zimmermann, B. Martín-López, And H. Von Wehrden. 2019. Research on the social perception of invasive species: a systematic literature review. NeoBiota 43:47-68.

KLUG, P., L. L. Wolfenbarger, and J. P. Mccarty. 2009. The nest predator community of grassland birds responds to agroecosystem habitat at multiple scales. Ecography 32:973-982.

Krasnov, B. R., G. I. Shenbrot, I. S. Khokhlova, and A. A. Degen. 2004. Flea species richness and parameters of host body, host geography and host "milieu." Journal of Animal Ecology 73:1121-1128.

LAfFerty, K. D., M. E. TorChin, AND A. M. KURIS. 2010. Parasite, The geography of host and Invasions. Pp. 191-203 in The Biogeography of Host-Parasite Interactions (B. R. Krasnov \& S. Morand, eds.). Oxford University Press, New York.

LAVILLA, E., E. RiChARD, AND G. SCROCCHI. 2000. Categorización de los anfibios y reptiles de la República Argentina. Asociación Herpetológica Argentina. San Miguel de Tucumán.

LAYNE, J. N., AND D. GLOVER. 1985. Activity patterns of the common long- nosed armadillo Dasyspus novemcinctus in South-Central Florida. Pp. 407-417 in The evolution and ecology of armadillos, sloth, and vermilinguas (G. G. Montgomery, ed.). Smithsonian Institution Press, Washington, DC.

Lindenfors, P., C. L. Nunn, K. E. Jones, A. A. Cunningham, W. Sechrest, And J. L. Gittleman. 2007. Parasite species richness in carnivores: Effects of host body mass, latitude, geographical range and population density. Global Ecology and Biogeography 16:496-509.

LOCKWOOD, J. L., P. CASSEY, AND T. BLACKBURN. 2005. The role of propagule pressure in explaining species invasions. Trends in Ecology and Evolution 20:223-228.

Loughry, W. J., AND C. M. Mcdonough. 2020. The Nine-Banded Armadillo. University of Oklahoma Press. 
LoVelL, S. J., S. F. StONE, AND L. Fernandez. 2006. The economic impacts of aquatic invasive species: A review of the literature. Agricultural and Resource Economics Review 35:195-208.

LUQUE, J. L., AND R. Poulin. 2008. Linking ecology with parasite diversity in Neotropical fishes. Journal of Fish Biology 72:189-204.

Lymbery, A. J., M. Morine, H. G. Kanani, S. J. Beatty, And D. L. Morgan. 2014. Co-invaders: The effects of alien parasites on native hosts. International Journal for Parasitology: Parasites and Wildlife 3:171-177.

Lyons, T. P., J. R. MILleR, D. M. DebInSKI, AND D. M. Engle. 2015. Predator identity influences the effect of habitat mangement on nest predation. Ecological Applications 25:In Press.

MANCINI, M., AND F. Grosman. 1998. Aspectos poblacionales del pejerrey Odontesthes bonariensis en el Embalse Río Tercero, Córdoba. Natura Neotropicalis. .

MARTIN, T. 1993. Nest Predation and Nest Sites. BioScience 43:523-532.

MARtínez, G., AND M. GutiéRrez. 2004. Tendencias en la explotación humana de la fauna durante el Pleistoceno final y Holoceno en la Región Pampeana (Argentina). Zooarchaeology of South America 1298:81-98.

MATTEUCCI, S. D. 2011. Ecorregión Estepa Patagónica. Pp. 549-654 in Ecorregiones y complejos ecosistémicos argentinos (J. Morello, S. D. Matteucci, A. F. Rodríguez \& M. Silva, eds.).

MAUReR, B. A., AND B. J. MCGILl. 2011. Measurement of species diversity. Pp. 55-65 in Biological Diversity: Frontiers in measurement and assessment. (B. J. Magurran, A. E. \& McGill, ed.). Oxford University Press.

MCDONOUgh, C. M., AND W. J. Loughry. 2008. Behavioral ecology of armadillos. The Biology of the Xenarthra 26:281-293. 
Mcdonough, C. M., Delaney M.J., P. Quoc L.E., M.S. Blackmore And W.J. Loughry. 2000.

Burrow characteristics and habitat associations of armadillos in Brazil and the United States of America. Revista de Biologia Tropical 48:109-120.

MCNAB, B.K. 1980. Energetics and the limits to a temperate distribution in armadillos. Journal of Mammalogy 61:606-627.

Mella-Romero, J., J. M. Ávila, F. R. Cortés, And C. M. Villouta. 2020. Registro de nado y evidencias indirectas del peludo patagónico Chaetophractus villosus ( Desmarest , 1804 ) en Aysén , Chile 48:47-51.

Mella-Romero, J., J. M. Ávila, F. R. Cortés, y C. M. VilloutA. 2020. Registro de nado y evidencias indirectas del peludo patagónico Chaetophractus villosus ( Desmarest , 1804 ) en Aysén , Chile 48:47-51.

Morales G. AND L. A. PINO. 1987. Parasitología cuantitativa. Fundación Fondo Editorial. Acta Científica Venezolana Eds. 332pp.

Moore, D. M. 1983. Flora of Tierra del Fuego. Anthony Nelson Ltd., Shrewsbury, Shropshire, U.K.

NAVONE, G. T. 1990. Estudio de la distribución, porcentaje y microecología de los parásitos de algunas especies de edentados argentinos. Studies on Neotropical Fauna and Environment 25:199-210.

Nunn, C. L., S. M. Altizer, W. SeChrest, And A. A. Cunningham. 2005. Latitudinal gradients of parasite species richness in primates. Diversity and Distributions 11:249-256.

PECK, S. B. 1986. Adult habits, larval morphology, and phylogenetic placement of Taurocerastes patagonicus Philippi ( Scarabaeidae : Geotrupinae ). Canadian Journal of Zoology 25:329-332.

Peredo, B. 1999. Bolivia's trade in hairy Armadillos. TRAFFIC Bulletin 18:41-45. 
Perrings, C. ET AL. 2002. Biological invasion risks and the public good: An economic perspective. Ecology and Society 6.

Poljak, S., J. Escobar, G. Deferrari, ANd M. Lizarralde. 2007. A new introduced mammal in Tierra del Fuego: the "large hairy armadillo" Chaetophractus villosus (Mammalia, Dasypodidae) in the Isla Grande island. Revista Chilena de Historia Natural 80:285294.

Poljak, S., J. SÁnCheZ, L. LANUSSE, AND M. S. LizARRALDE. 2020. Anthropogenic invaders: Historical biogeography, current genetic status and distribution range of the "peludo" Chaetophractus villosus (Xenarthra) in Patagonia and Tierra del Fuego, southern South America. Mammalia.

Poljak, S., V. Confalonieri, M. Fasanella, M. Gabrielli and M.S. Lizarralde. 2010. Phylogeography of the armadillo Chaetophractus villosus (Dasypodidae Xenarthra): Post-glacial range expansion from Pampas to Patagonia (Argentina). Molecular Phylogenetics and Evolution 55:38-46. Elsevier Inc.

POSADAS, P. 2012. Species composition and geographic distribution of Fuegian Curculionidae (Coleoptera: Curculionoidea). Zootaxa 36:1-36.

Poulin, R. 1995. Phylogeny, ecology, and the richness of parasite communities in vertebrates. Ecological Monographs 65:283-302.

POULIN, R., AND B. R. KRASNOV. 2010. Similarity and variability of parasite assemblages across geographical space. Pp. 115-127 in The Biogeography of Host-Parasite Interactions (S. Morand \& B. R. Krasnov, eds.). Oxford University Press, New York.

PoULIN, R., AND T. L. F. LEUNG. 2011. Latitudinal gradient in the taxonomic composition of parasite communities. Journal of Helminthology 85:228-233.

PREISSER, W. 2019. Latitudinal gradients of parasite richness: a review and new insights from helminths of cricetid rodents. Ecography 42:1315-1330. 
Pyke, G. H., H. R. Pulliam, And E. L. Charnov. 1977. Optimal foraging: a selective review of theory and tests. The Quarterly Review of Biology 52:137-154.

Qgis DeVelopment Team. 2017. QGIS Geographic Information System. Open Source Geospatial Foundation Project. <http://qgis.osgeo.org>.

Rader, M. J., T. W. Teinert, L. A. Brennan, F. Hernández, N. J. Silvy, And X. Ben Wu. 2007. Identifying predators and nest fates of bobwhites in southern Texas. Journal of Wildlife Management 71:1626-1630

R CORE TEAm. 2017. R: A language and environment for statistical computing. R Foundation for Statistical Computing, Vienna, Austria. <https://www.r-project.org/>.

REDFORD, K.H. 1985. Food habits of armadillos (XENARTHRA: DASYPODIDAE). Pp. 429-437, in: The evolution and ecology of armadillos, sloths and vermilinguas (G. Montgomery, ed.). Smithsonian Institution Press, Washington \& London.

RIBICICH, M. ET AL. 2010. Trichinella infection in wild animals from endemic regions of Argentina. Parasitology Research 107:377-380.

RimoldI, P.G. y A.M. ABBA. 2013. Nuevos datos de presencia de Chaetophractus villosus en la cuenca del Río Carcarañá, Sur de la Provincia de Santa Fe, Argentina. Edentata 14:1-8.

RIOS, T. A. 2020. Estudios parasitológicos en xenartros (MAMMALIA: XENARTHRA) del Chaco argentino. Universidad Nacional de La Plata.

Rodríguez, M., A. France y M. Gerding. 2004. Evaluación de dos cepas del hongo Metarhizium anisopliae var. Anisopliae (metsh.) para el control de larvas de gusano blanco Hylamorpha legans Burm. (COLEOPTERA: SCARABAEIDAE). Agricultura Técnica 64:17-24.

Rodríguez, M., A. France, y M. Gerding. 2004. Evaluación de dos cepas del hongo Metarhizium anisopliae var. Anisopliae (metsh.) para el control de larvas de gusano 
blanco Hylamorpha legans Burm. (COLEOPTERA: SCARABAEIDAE). Agricultura Técnica 64:17-24.

Ruffino, L., J. C. Russell, B. PISANu, S. CAUt, ANd E. VidAL. 2011. Low individual-level dietary plasticity in an island-invasive generalist forager. Population Ecology 53:535-548.

Salo, P., E. Korpimaki, P. B. Banks, M. Nordstrom, AND C. R. DickMan. 2007. Alien predators are more dangerous than native predators to prey populations. Proceedings of the Royal Society B: Biological Sciences 274:1237-1243.

SÁnCHEZ, J., AND M. S. LizARRALDE. 2019. Ctenomys magellanicus. Categorización 2019 de los mamíferos de Argentina según su riesgo de extinción. Lista Roja de los mamíferos de Argentina. (SAyDS-SAREM, ed.). SAyDS-SAREM.

SAnguinetti, J. ET Al. 2014. Manejo de especies exóticas invasoras en Patagonia, Argentina: Priorización, logros y desafíos de integración entre ciencia y gestión identificados desde la Administración de Parques Nacionales. Ecologia Austral 24:183-192.

Shackleton, R. T., B. M. H. Larson, A. Novoa, D. M. Richardson, and C. A. Kull. 2019. The human and social dimensions of invasion science and management. Journal of Environmental Management 229:1-9.

SiH, A., AND B. Christensen. 2001. Optimal diet theory: When does it work, and when and why does it fail? Animal Behaviour 61:379-390.

Sikes, R. S., And A. C. And U. C. Of The A. S. Of Mammalogists. 2016. 2016 Guidelines of the American Society of Mammalogists for the use of wild mammals in research and education. Journal of mammalogy 97:663-688.

Soininen, J., R. Mcdonald, ANd H. Hillebrand. 2007. The distance decay of similarity in ecological communities. Ecography 30:3-12.

STEHR, F. W. 1987. Inmature Insects. Vol I. Kendall/Hunt publishing company, Dubuque, Iowa, USA. 
STEHR, F. W. 1991. Inmature Insects.Vol II. Kendall/Hunt publishing company, Dubuque, Iowa, USA.

SUPERINA, M. AND P. BOILY. 2007. Hibernation and daily torpor in an armadillo, the pichi (Zaedyus pichiy). Comparative Biochemistry and Physiology - A Molecular and Integrative Physiology 148:893-898.

SUPERINA, M. ET AL. 2019. Zaedyus pichiy. Categorización 2019 de los mamíferos de Argentina según su riesgo de extinción. Lista Roja de los mamíferos de Argentina.

TAPIAS M, M., Y V. IDROVO C. 2013. Aportes de la etonobotánica al estudio de las invasiones biológicas. Casos en la región rioplatense (Argentina). Historia Natural 3:61-76.

TAULMAN, J.F. AND L.W. RoBBINS. 1996. Recent range expansion and distributional limits of the nine-banded armadillo (Dasypus novemcinctus) in the United States. Journal of Biogeography 23:635-648.

Teta, P., A.M. AbBa, G. Cassini, D. Flores, C. Galliari, S. LuCero, et Al. 2018. Lista revisada de los mamíferos de Argentina. Mastozoología Neotropical.

THOMPSON, D.W.J. 2002. Interpretation of Recent Southern Hemisphere Climate Change. Science 296:895-899.

Torchin, M. E., K. D. Lafferty, A. P. Dobson, V. J. Mckenzie, And A. M. KuRIS. 2003. Introduced species and their missing parasites 421:628-630.

Villalba, R., M. Masiokas, T. KitZberger And J. Boninsegna. 2005. Biogeographical consequences of recent climate changes in the southern Andes of Argentina. Pp. 157-168, in: Global changes and mountain regions (U Huber \& M Reasoner, eds.). Mountain Research Initiative, Switzerland.

WITH, K.A. 2002. The landscape ecology of invasive species. Conservation Biology 16:11921203. 


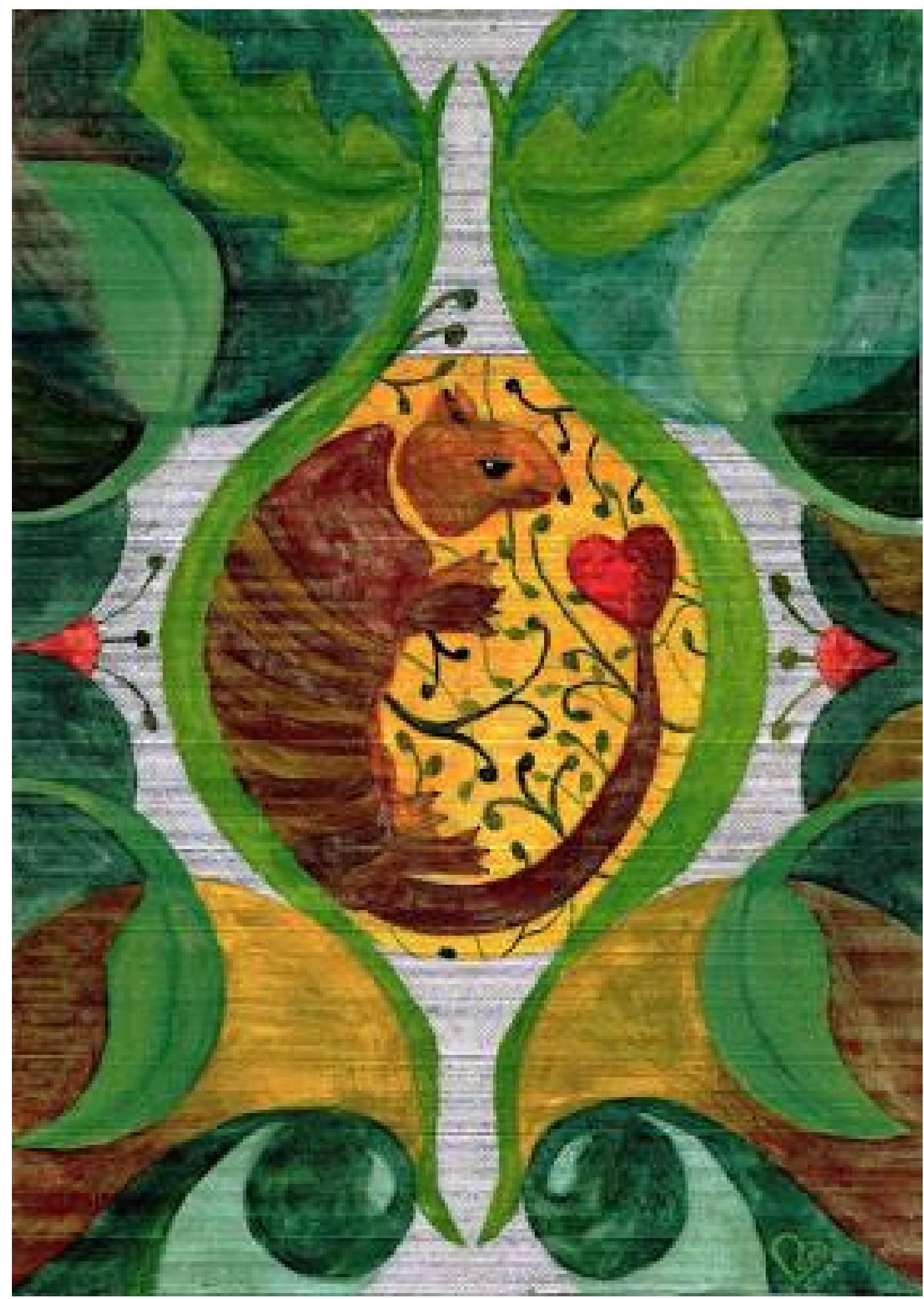

Quirquincho

Obra de Bon Core 